\title{
Pseudodimeric Complexes of an (18-Crown-6)stilbene with Styryl Dyes Containing an Ammonioalkyl Group: Synthesis, Structure, and Stereospecific [2 + 2] Cross-Photocycloaddition
}

Timofey P. Martyanov, ${ }^{\mathrm{a} *}$ Evgeny N. Ushakov, ${ }^{\mathrm{a}, \mathrm{b}}$ Vyacheslav N. Nuriev, ${ }^{\mathrm{b}, \mathrm{c}}$ Nadezhda A. Aleksandrova, ${ }^{\mathrm{b}}$ Sergey K. Sazonov, ${ }^{\mathrm{b}}$ Artem I. Vedernikov, ${ }^{\mathrm{b}}$ Lyudmila G. Kuz'mina, ${ }^{\mathrm{d}}$ Lubov S. Klimenko, ${ }^{\mathrm{e}}$ Elena G. Martyanova, ${ }^{\mathrm{a}}$ and Sergey P. Gromov ${ }^{\mathrm{b}, \mathrm{c}, *}$

${ }^{a}$ Institute of Problems of Chemical Physics, Russian Academy of Sciences, Chernogolovka, Moscow Region, 142432, Russian Federation

b Photochemistry Center of RAS, FSRC "Crystallography and Photonics", Russian Academy of Sciences, Novatorov str. 7A-1, Moscow, 119421, Russian Federation

${ }^{c}$ Department of Chemistry, M. V. Lomonosov Moscow State University, Leninskie Gory 1-3, Moscow, 119991, Russian Federation

${ }^{d}$ N.S. Kurnakov Institute of General and Inorganic Chemistry, Russian Academy of Sciences, Leninskiy prosp. 31, Moscow, 119991, Russian Federation

${ }^{\mathrm{e}}$ Yugra State University, Khanty-Mansiysk, 628012, Russian Federation

* Corresponding authors. E-mail addresses: martyanov.t@gmail.com (T.P. Martyanov), spgromov@mail.ru (S.P. Gromov). 


\section{Table of Contents}

I Figure S1: Spectrophotometric titration data for the $(E)-\mathbf{1}-\mathrm{EtNH}_{3} \mathrm{ClO}_{4}$ system

II Figures S2-S4: ${ }^{1} \mathrm{H}$ NMR spectra of dye $(E)$-2b and complex $(E)-\mathbf{1} \cdot(E)$-2b before and after irradiation by light, stilbene $(E)-1$

III Figures S5-S10: Spectrophotometric data for measurements of quantum yields

IV Figures S11-S13: Fluorescence decay kinetics for dyes $(E)-\mathbf{2 a}, \mathbf{b}$ and equimolar mixture of $(E)-\mathbf{2 a}$ and $(E)-\mathbf{1}$

V Figures S14-S26: Changes in the ${ }^{1} \mathrm{H}$ NMR spectra upon complexation of compound $(E)-\mathbf{1}$ with dye $(E)$-2a, NMR spectra of cyclobutane $\mathbf{6}$

VI Figures S27, S28 and Table S1: X-ray crystallography data

VII Figure S29: Notation for conformers of $(E)-\mathbf{1},(E)-\mathbf{2 a}, \mathbf{b}$, and $(E)-\mathbf{1} \cdot(E)-\mathbf{2 a}, \mathbf{b}$.

VIII Tables S2-S4, Figure S30: Gibbs free energies in solution for different conformers of $(E)-\mathbf{1},(E)-\mathbf{2 a}, \mathbf{b}$, and $(E)-\mathbf{1} \cdot(E)-\mathbf{2 a}, \mathbf{b}$, as derived from DFT/SMD calculations S22

IX Tables S5-S8: TDDFT calculations S24

X Figures S31-S37: NMR and ESI mass spectra of synthesized compounds S28

XI Table S9: Cartesian coordinates from DFT calculations in solution S32 
I Figure S1: Spectrophotometric titration data for the (E)-1- $\mathrm{EtNH}_{3} \mathrm{ClO}_{4}$ system

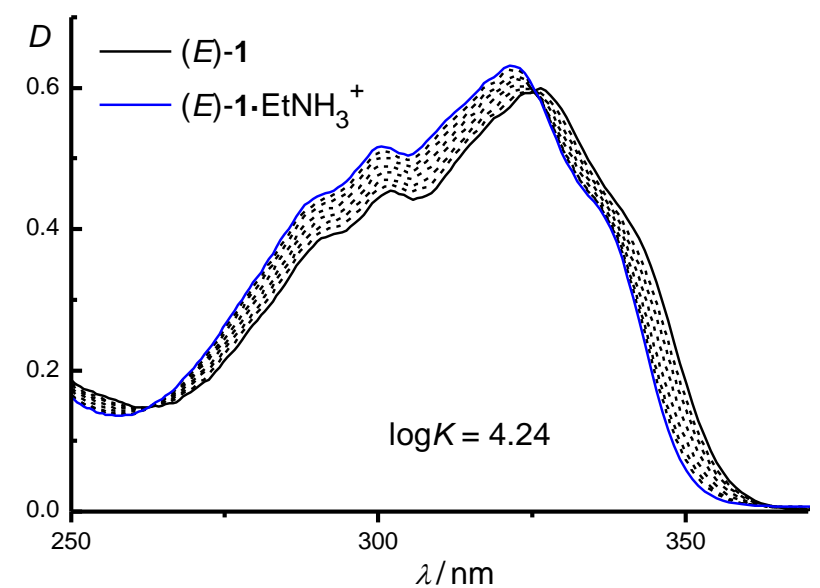

Figure S1. Spectrophotometric titration data for the $(E)-\mathbf{1}-\mathrm{EtNH}_{3} \mathrm{ClO}_{4}$ system in $\mathrm{MeCN}(1 \mathrm{~cm}$ cell), $C_{(E)-1}=2.0 \times 10^{-5} \mathrm{M}$; the concentration of ethylammonium perchlorate varies from 0 to $1 \times 10^{-3} \mathrm{M}$. 
II Figures S2-S4: ${ }^{1} \mathrm{H}$ NMR spectra of dye $(E)-\mathbf{2 b}$ and complex $(E)-\mathbf{1} \cdot(E)$-2b before and after irradiation by light, stilbene $(E)-1$

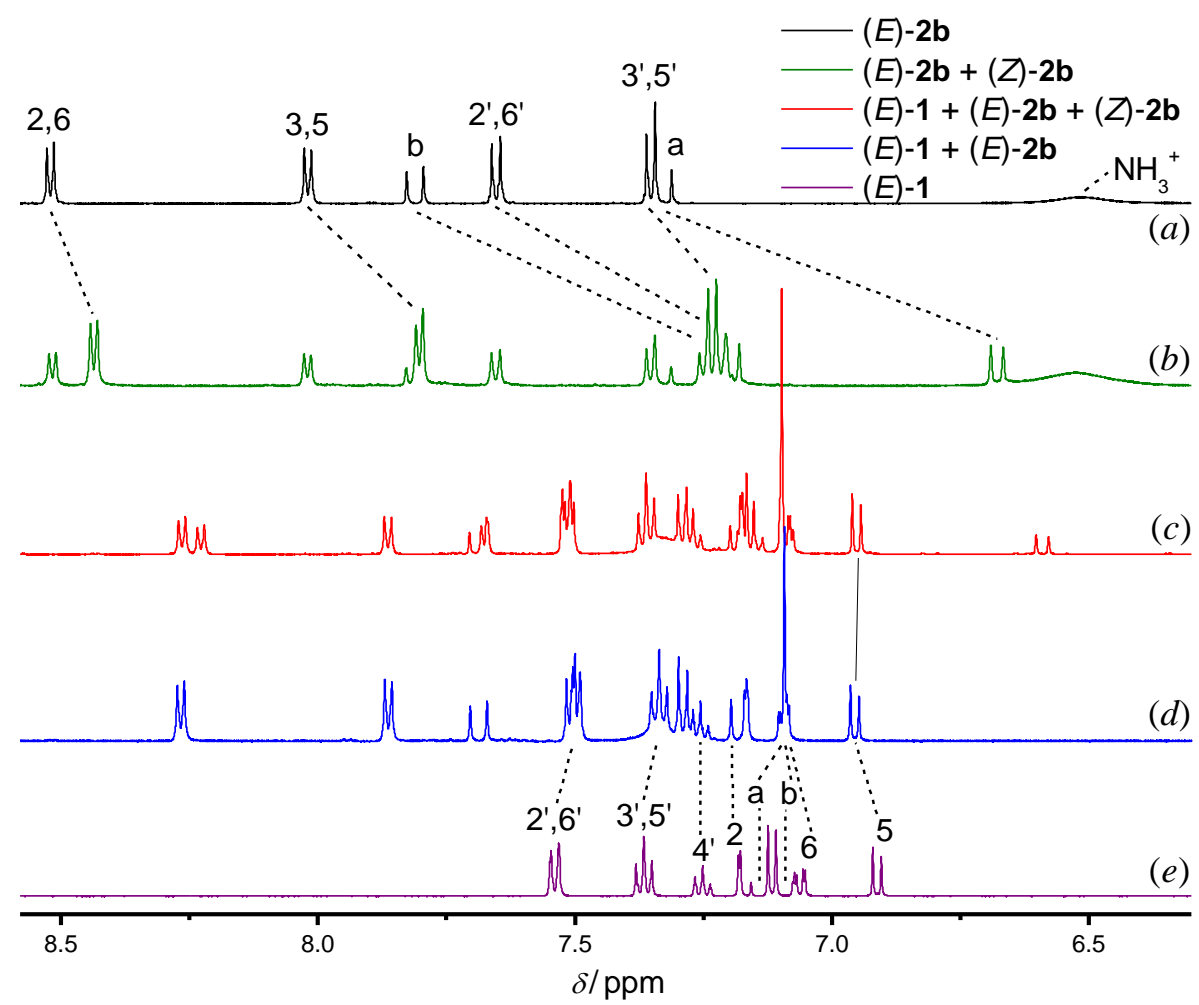

Figure S2. ${ }^{1} \mathrm{H}$ NMR $(296 \mathrm{~K}, 500 \mathrm{MHz})$ spectra (aromatic proton region) of $(a)$ dye $(E)-\mathbf{2 b},(b)$ dye $(E)$-2b exposed to light $(405 \mathrm{~nm}),(c)$ a 1:1 mixture of $(E)-\mathbf{1}$ and $(E)-\mathbf{2 b}$ exposed to light (405 nm) for $300 \mathrm{~s},(d)$ a $1: 1$ mixture of $(E)-\mathbf{1}$ and $(E)-\mathbf{2 b},(e)$ compound $(E)-\mathbf{1}: 2 \times 10^{-3} \mathbf{M}, \mathrm{MeCN}-d_{3}$. 


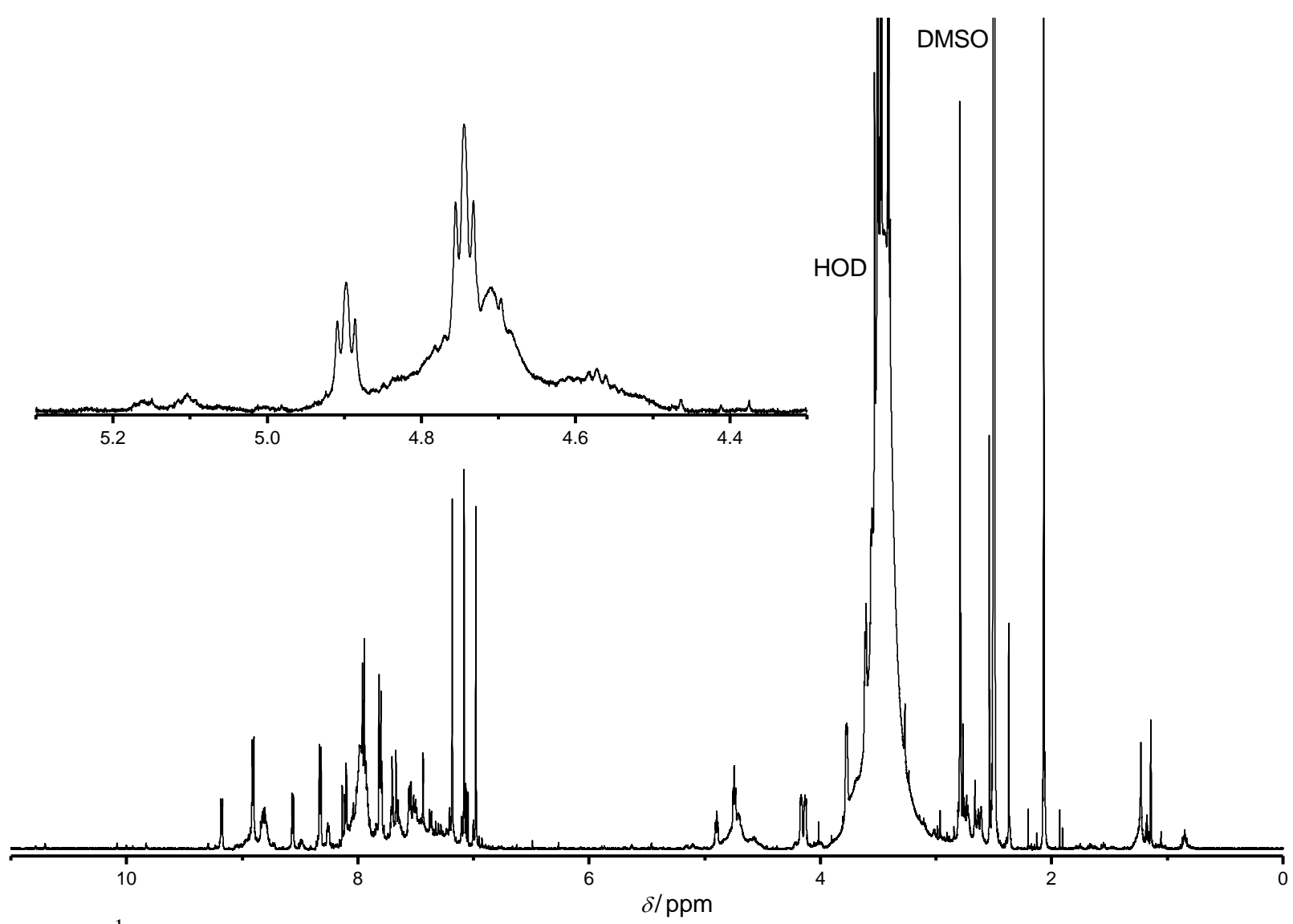

Figure S3. ${ }^{1} \mathrm{H}$ NMR $(296 \mathrm{~K}, 500 \mathrm{MHz})$ spectrum of a 1:1 mixture of $(E)-\mathbf{1}$ and $(E)$-2a exposed to light $(405 \mathrm{~nm})$ for $50 \mathrm{~h}: C=2 \times 10^{-3} \mathrm{M}$, DMSO- $d_{6}$.

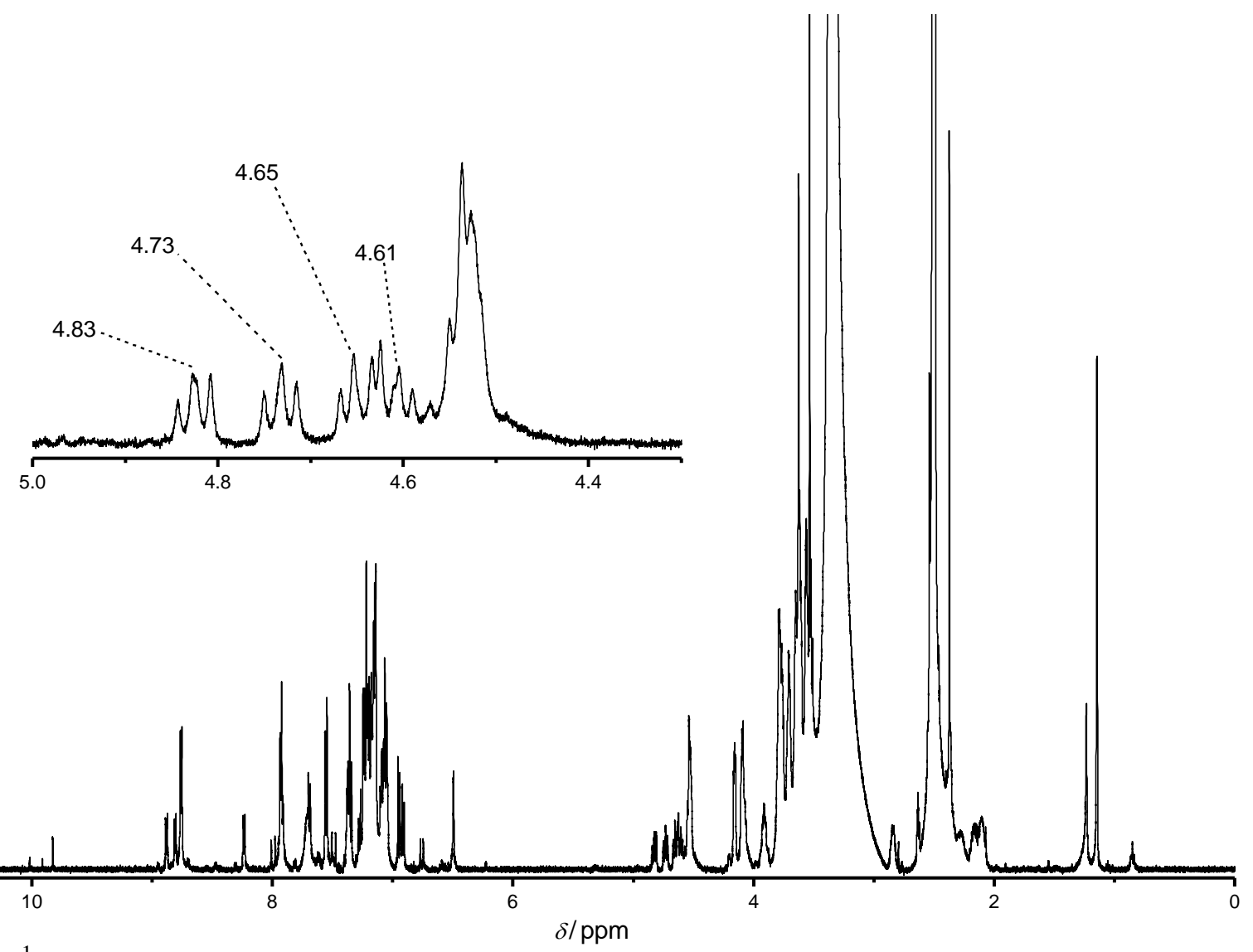

Figure S4. ${ }^{1} \mathrm{H}$ NMR $(296 \mathrm{~K}, 500 \mathrm{MHz})$ spectrum of a 1:1 mixture of $(E)-\mathbf{1}$ and $(E)$-2b exposed to light $(405 \mathrm{~nm})$ for $40 \mathrm{~h}: C=2 \times 10^{-3} \mathrm{M}$, DMSO- $d_{6}$. 
III Figures S5-S10: Spectrophotometric data for measurements of quantum yields

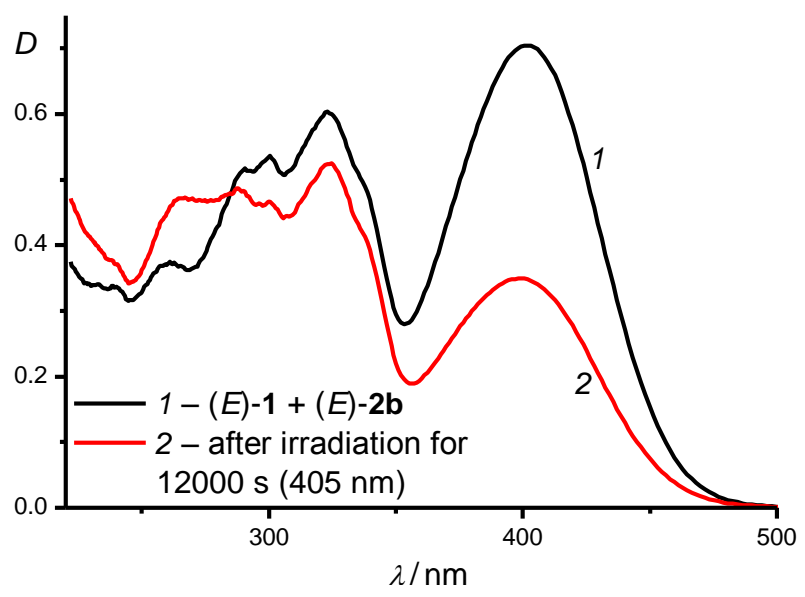

Figure S5. (1) Absorption spectrum of a mixture of $(E)-\mathbf{1}$ and $(E)-\mathbf{2 b}$ in $\mathrm{MeCN}\left(C_{\mathbf{1}}=9.86 \times 10^{-5} \mathrm{M}, C_{\mathbf{2 b}}\right.$ $\left.=9.97 \times 10^{-5} \mathrm{M}, l=0.2 \mathrm{~cm}\right) ;(2)$ after irradiation at $\lambda=405 \mathrm{~nm}$ with light intensity of $3.14 \times 10^{-9}$ $\mathrm{mol} \cdot \mathrm{cm}^{-2} \cdot \mathrm{s}^{-1}$ for $12000 \mathrm{~s}$.

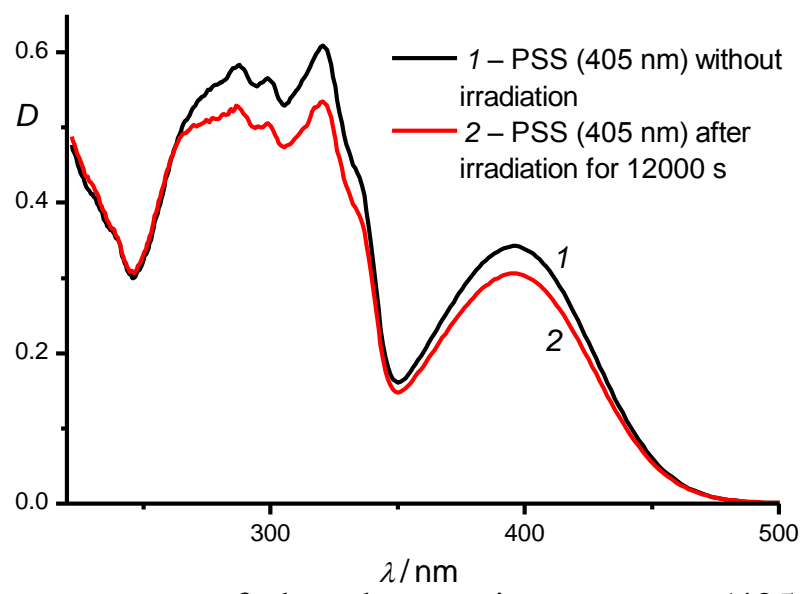

Figure S6. (1) Absorption spectrum of the photostationary state $(405 \mathrm{~nm})$ after the addition of $\mathrm{Ba}\left(\mathrm{ClO}_{4}\right)_{2}\left(5 \times 10^{-3} \mathrm{M}\right)$ to a mixture of $(E)-\mathbf{1}$ and $(E)-\mathbf{2 b}$ in a MeCN solution $\left(C_{\mathbf{1}}=9.86 \times 10^{-5} \mathrm{M}, C_{\mathbf{2 b}}=\right.$ $\left.9.97 \times 10^{-5} \mathrm{M}, l=0.2 \mathrm{~cm}\right)$; (2) absorption spectrum of the photostationary state (PSS) obrained at $\lambda=$ $405 \mathrm{~nm}$ after the addition of $\mathrm{Ba}\left(\mathrm{ClO}_{4}\right)_{2}\left(5 \times 10^{-3} \mathrm{M}\right)$ to irradiated $(405 \mathrm{~nm} ; 12000 \mathrm{~s})$ mixture of $(E)-\mathbf{1}$ and $(E)-\mathbf{2 b}$ in a MeCN solution $\left(C_{\mathbf{1}}=9.86 \times 10^{-5} \mathrm{M}, C_{\mathbf{2 b}}=9.97 \times 10^{-5} \mathrm{M}, l=0.2 \mathrm{~cm}\right)$. 


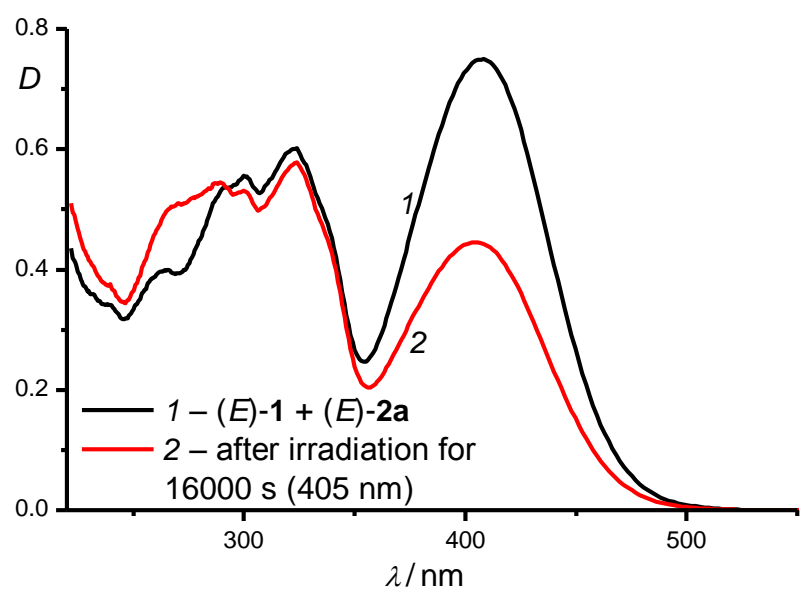

Figure S7. (1) Absorption spectrum of a mixture of $(E)-\mathbf{1}$ and $(E)-\mathbf{2 a}$ in a MeCN solution $\left(C_{\mathbf{1}}=\right.$ $\left.9.91 \times 10^{-5} \mathrm{M}, C_{2 \mathrm{a}}=9.94 \times 10^{-5} \mathrm{M}, l=0.2 \mathrm{~cm}\right)$; (2) after irradiation at $\lambda=405 \mathrm{~nm}$ with light intensity of $3.07 \times 10^{-9} \mathrm{~mol} \cdot \mathrm{cm}^{-2} \cdot \mathrm{s}^{-1}$ for $16000 \mathrm{~s}$.

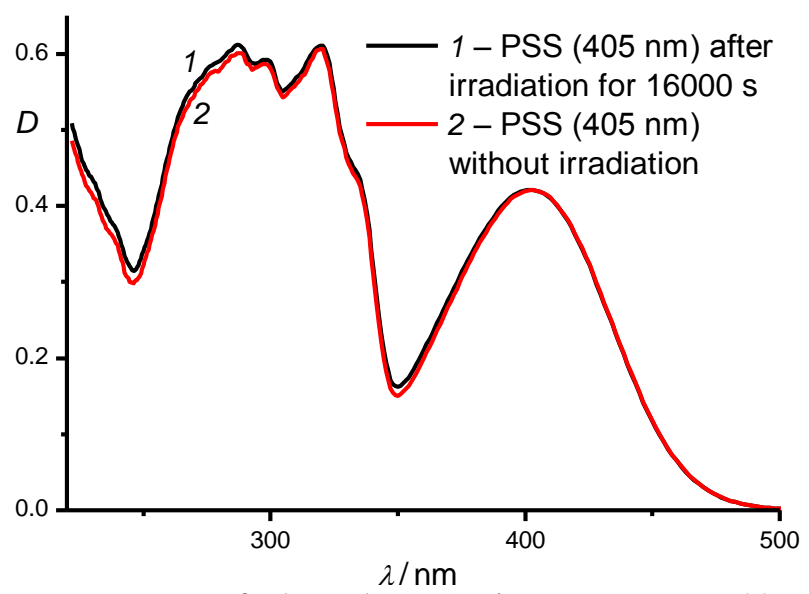

Figure S8. (1) Absorption spectrum of the photostationary state $(405 \mathrm{~nm})$ after the addition of $\mathrm{Ba}\left(\mathrm{ClO}_{4}\right)_{2}\left(5 \times 10^{-3} \mathrm{M}\right)$ to a mixture of $(E)-\mathbf{1}$ and $(E)-\mathbf{2 a}$ in a MeCN solution $\left(C_{\mathbf{1}}=9.91 \times 10^{-5} \mathrm{M}, C_{\mathbf{2 a}}=\right.$ $\left.9.94 \times 10^{-5} \mathrm{M}, l=0.2 \mathrm{~cm}\right)$; (2) absorption spectrum of the photostationary state $(405 \mathrm{~nm})$ after the addition of $\mathrm{Ba}\left(\mathrm{ClO}_{4}\right)_{2}\left(5 \times 10^{-3} \mathrm{M}\right)$ to a solution of irradiated $(405 \mathrm{~nm}, 16000 \mathrm{~s})$ mixture of $(E)-\mathbf{1}$ and $(E)-\mathbf{2 a}$ in $\mathrm{MeCN}\left(C_{\mathbf{1}}=9.91 \times 10^{-5} \mathrm{M}, C_{\mathbf{2 b}}=9.94 \times 10^{-5} \mathrm{M}, l=0.2 \mathrm{~cm}\right)$. 


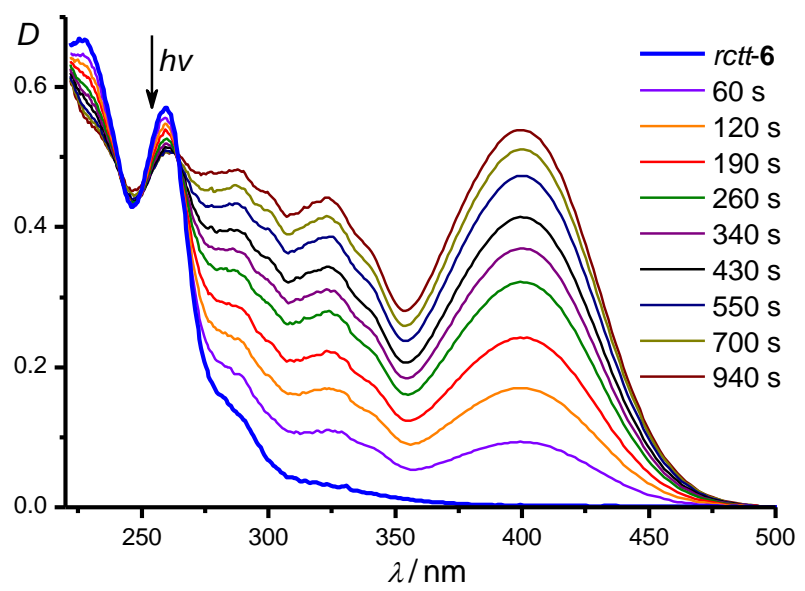

Figure S9. Spectrophotometric data on the steady-state photolysis of cyclobutane rctt-6 with $254 \mathrm{~nm}$ light in MeCN $(1 \mathrm{~cm}$ cell $), C_{r c t t-6}=2.7 \times 10^{-5} \mathrm{M}$.
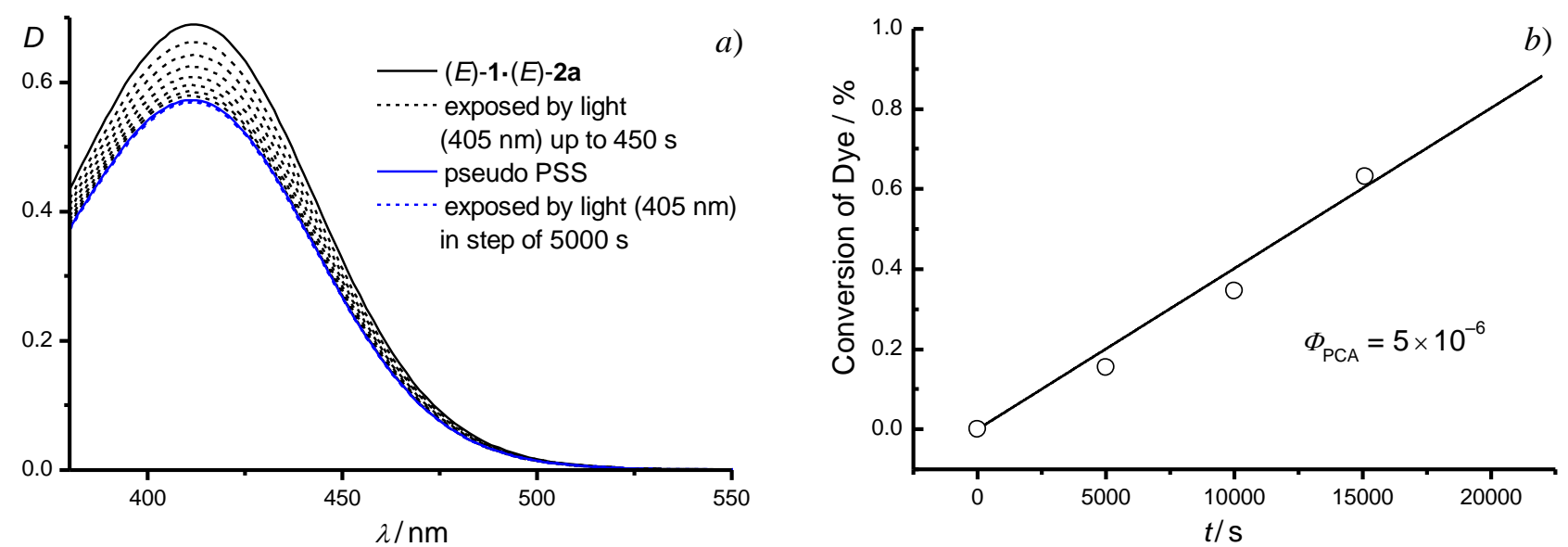

Figure S10. $a)$ Spectrophotometric data on the steady-state photolysis of complex $(E)-\mathbf{1} \cdot(E)-\mathbf{2 a}$ with $405 \mathrm{~nm}$ light: $\mathrm{MeCN}, 1 \mathrm{~cm}$ cell, $C_{(E)-1}=7.0 \times 10^{-4} \mathrm{M}, C_{(E)-2 \mathrm{a}}=2.1 \times 10^{-5} \mathrm{M}$, light intensity of $3.94 \times 10^{-9}$ mol cm $\left.\mathrm{cm}^{-2} \mathrm{~s}^{-1} ; b\right)$ percentage of conversion of complexed dye $(E)-\mathbf{2 a}$ as a function of the irradiation time: the solid curve is based on global fitting of the experimental data to a kinetic equation for irreversible unimolecular photoreactions. 
IV Figures S11-S13: Fluorescence decay kinetics for dyes $(E)-\mathbf{2 a}, \mathbf{b}$ and equimolar mixture of $(E)-\mathbf{2 a}$ and $(E)-\mathbf{1}$

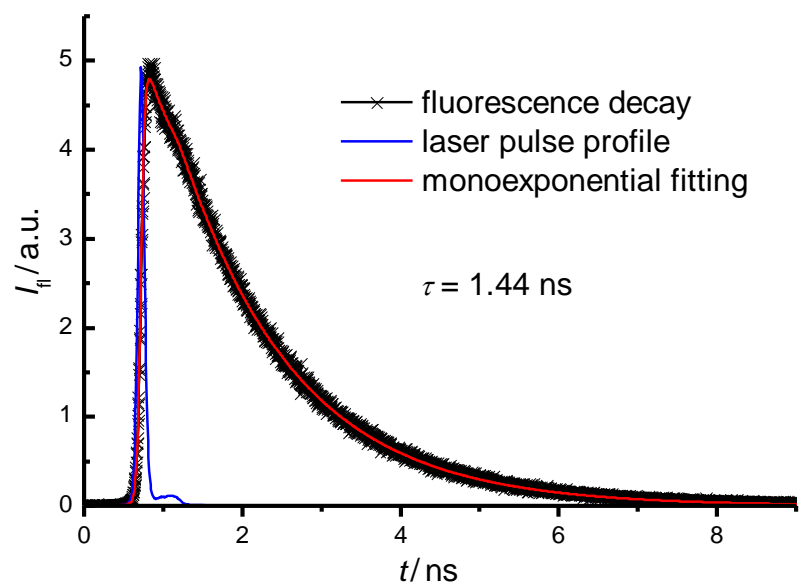

Figure S11. Fluorescence decay kinetics for dye $(E)$-2a in $\mathrm{MeCN}$ : excitation with 375-nm laser pulse; observation at 540,580, and $620 \mathrm{~nm}$; the blue curve is the laser pulse profile; the solid red curve is from global fitting to the monoexponential model $I_{\mathrm{fl}}(\lambda, t)=A(\lambda) \times \exp (-t / \tau)$, with $\tau=1.44 \mathrm{~ns}$.

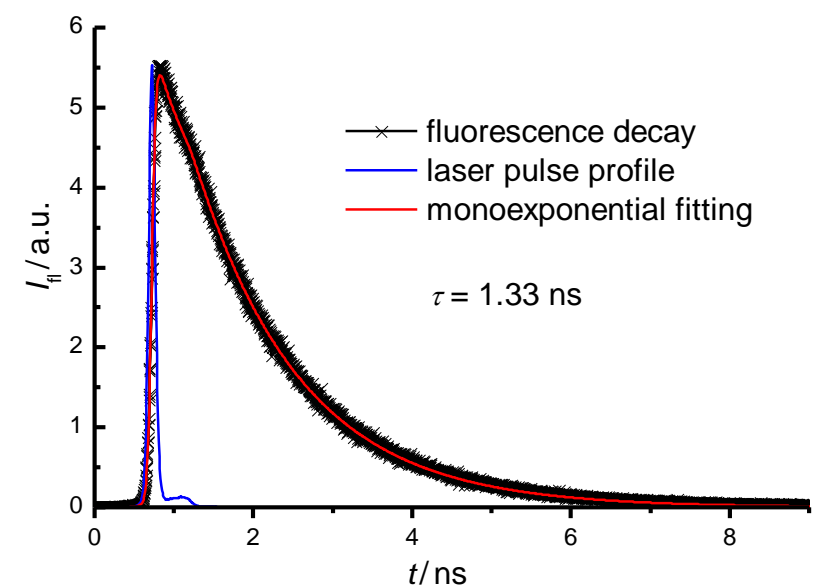

Figure S12. Fluorescence decay kinetics for dye $(E)$-2b in $\mathrm{MeCN}$ : excitation with 375-nm laser pulse; observation at 532, 572, and $612 \mathrm{~nm}$; the blue curve is the laser pulse profile; the solid red curve is from global fitting to the monoexponential model $I_{\mathrm{fl}}(\lambda, t)=A(\lambda) \times \exp (-t / \tau)$, with $\tau=1.33 \mathrm{~ns}$.

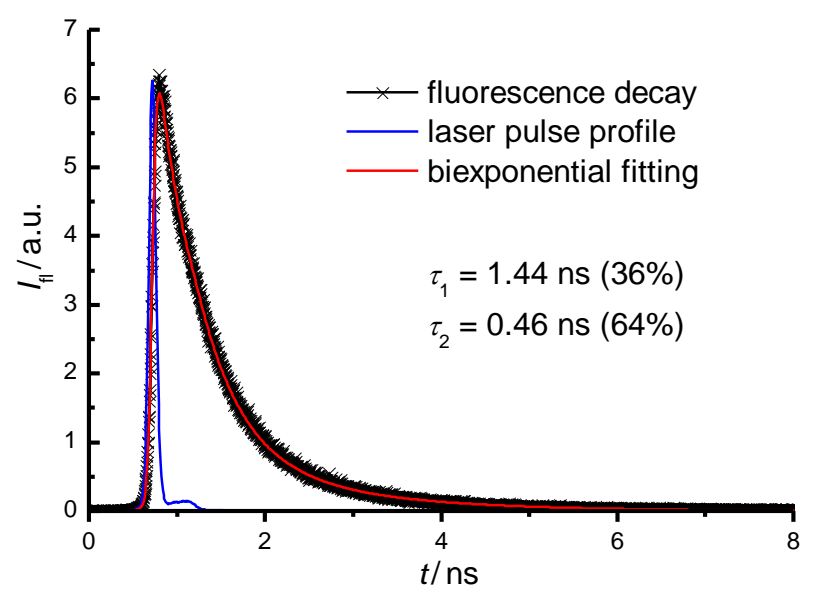

Figure S13. Fluorescence decay kinetics for mixture of dye $(E)-2 \mathbf{a}\left(2 \times 10^{-5} \mathrm{M}\right)$ and stilbene $(E)-\mathbf{1}$ $\left(7 \times 10^{-4} \mathrm{M}\right)$ in MeCN: excitation with $375-\mathrm{nm}$ laser pulse; observation at 540, 580, and $620 \mathrm{~nm}$; the blue curve is the laser pulse profile; the solid red curve is from global fitting to the biexponential model $I_{\mathrm{fl}}(\lambda, t)=A_{1}(\lambda) \times \exp \left(-t / \tau_{1}\right)+A_{2}(\lambda) \times \exp \left(-t / \tau_{2}\right)$, with $\tau_{1}=1.44 \mathrm{~ns}$ and $\tau_{2}=0.46 \mathrm{~ns}$. The integral contributions of components are shown in parentheses. 


\section{About radiative lifetimes}

A radiative lifetime $\tau_{0}$ of a spontaneous transition between electronic states in the case of a symmetric absorption band is

$$
\tau_{0}=\frac{3.5 \times 10^{8} g}{v_{\max }^{2} \varepsilon_{\max } \Delta v_{1 / 2}},
$$

where $g$ is the degree of degeneracy (multiplicity) of a given state, equal to 1 for singlet-singlet transitions and 3 for singlet-triplet transitions; $v_{\max }$ is the wavenumber at the absorption band maximum, $\mathrm{cm}^{-1} ; \varepsilon-$ is the molar absorption coefficient at $v_{\max }, \mathrm{M}^{-1} \mathrm{~cm}^{-1} ; \Delta v_{1 / 2}$ is the full width at half maximum $\left(v_{\max }\right), \mathrm{cm}^{-1}$. For the complex, the $\Delta v_{1 / 2}$ value was obtained by approximating the longwavelength absorption band by two lognormal functions.

For free styryl dye $(E)-\mathbf{2 b}$, we get:

$$
\tau_{0}=3.4 \mathrm{~ns} .
$$

For the complex $(E)-\mathbf{1} \cdot(E)-\mathbf{2 b}$, we get:

$$
\tau_{0}=3.9 \mathrm{~ns} .
$$

As one can see, the radiative lifetime of the dye hardly changes during complexation. 
V Figures S14-S26: Changes in the ${ }^{1} \mathrm{H}$ NMR spectra upon complexation of compound $(E)-\mathbf{1}$ with dye $(E)$-2a, NMR spectra of cyclobutane $\mathbf{6}$

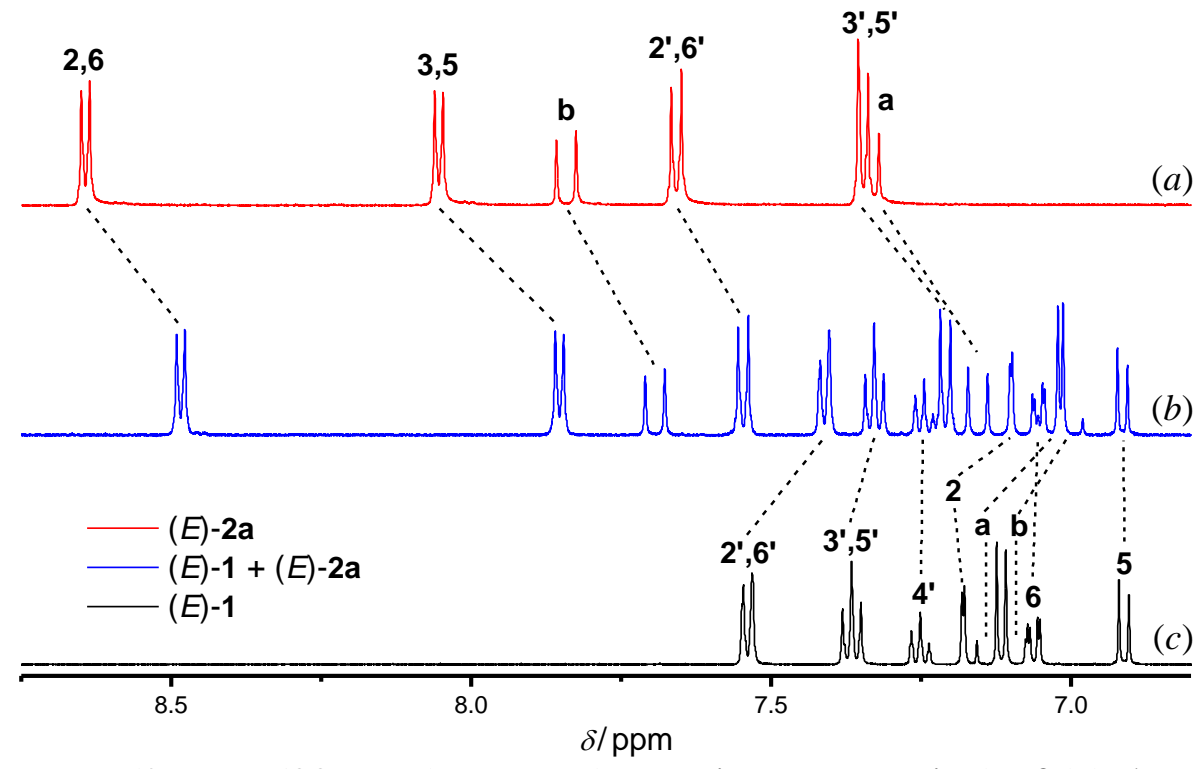

Figure S14. ${ }^{1} \mathrm{H}$ NMR $(296 \mathrm{~K}, 500 \mathrm{MHz})$ spectra (aromatic proton region) of $(a)$ dye $(E)$-2a, $(b)$ a 1:1 mixture of $(E)-\mathbf{1}$ and $(E)$-2a, and $(c)$ compound $(E)-\mathbf{1}: 2 \times 10^{-3} \mathrm{M}, \mathrm{MeCN}-d_{3}-\mathrm{D}_{2} \mathrm{O}(98: 2, \mathrm{v} / \mathrm{v})$.

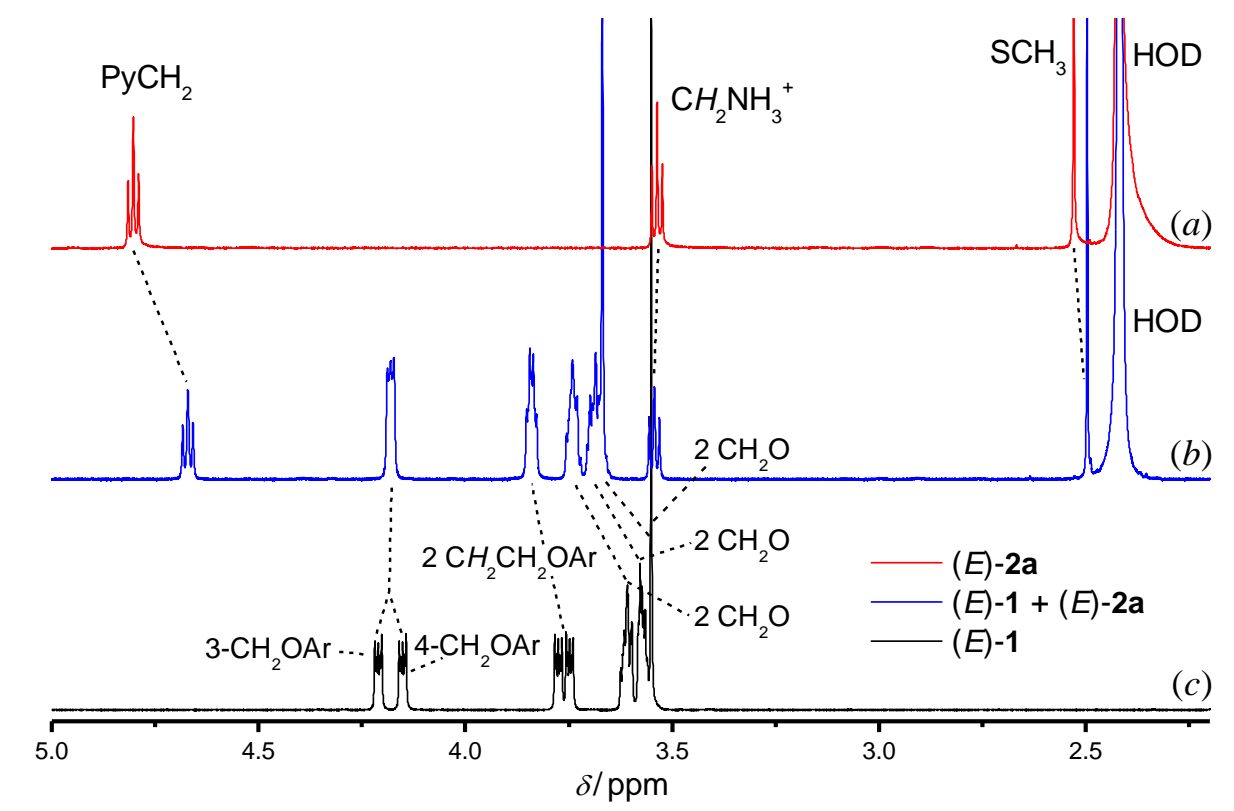

Figure S15. ${ }^{1} \mathrm{H}$ NMR $(296 \mathrm{~K}, 500 \mathrm{MHz})$ spectra (aliphatic proton region) of $(a)$ dye $(E)$-2a, $(b)$ a 1:1 mixture of $(E)$-1 and $(E)$-2a, and $(c)$ compound $(E)-\mathbf{1}: 2 \times 10^{-3} \mathrm{M}, \mathrm{MeCN}-d_{3}-\mathrm{D}_{2} \mathrm{O}(98: 2, \mathrm{v} / \mathrm{v})$. 


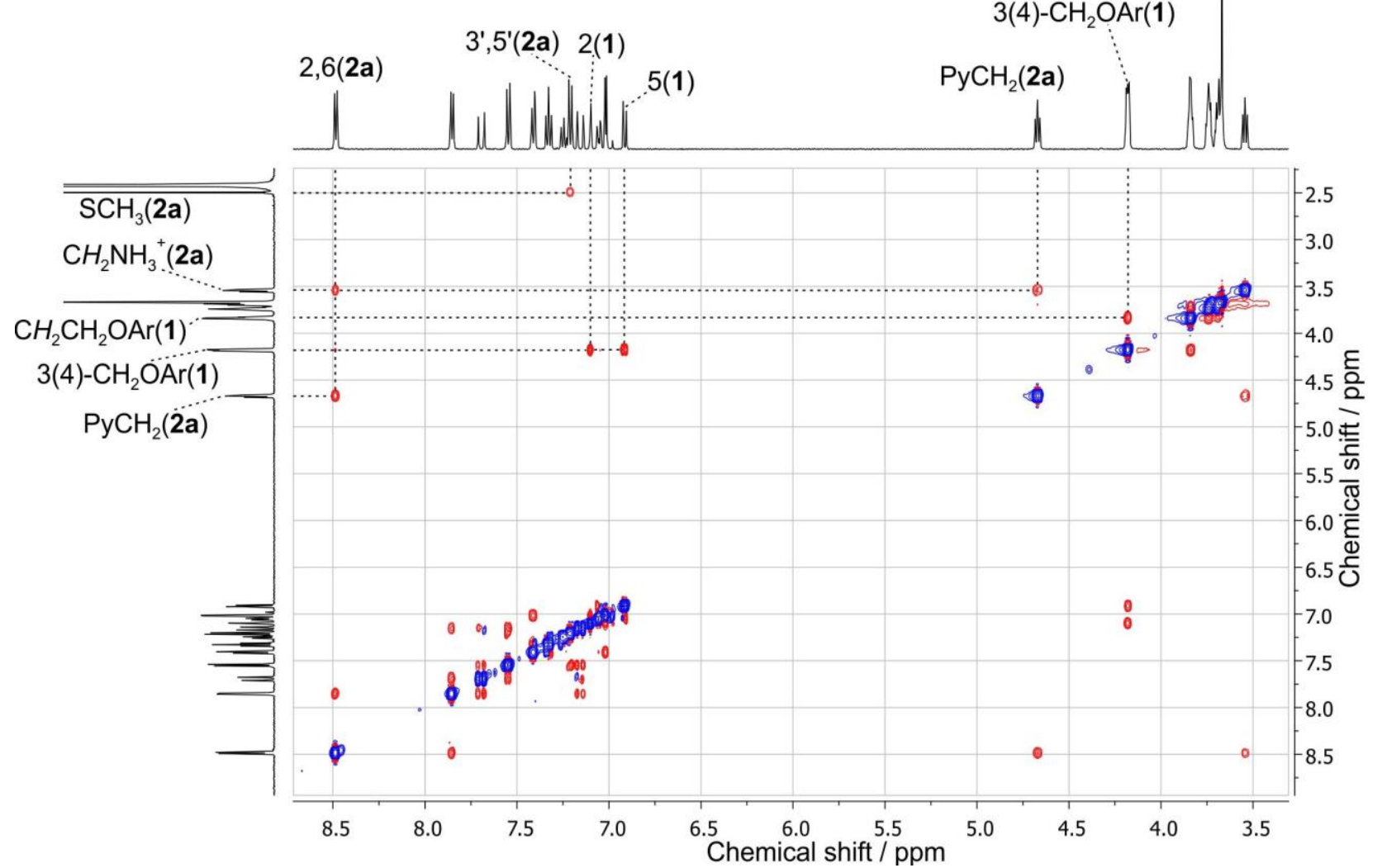

Figure S16. NOESY $(296 \mathrm{~K}, 500 \mathrm{MHz})$ spectrum of equimolar $\left(2 \times 10^{-3} \mathrm{M}\right)$ mixture of $(E)-\mathbf{1}$ and $(E)$ 2a in $\mathrm{MeCN}-d_{3}-\mathrm{D}_{2} \mathrm{O}(98: 2, \mathrm{v} / \mathrm{v})$.

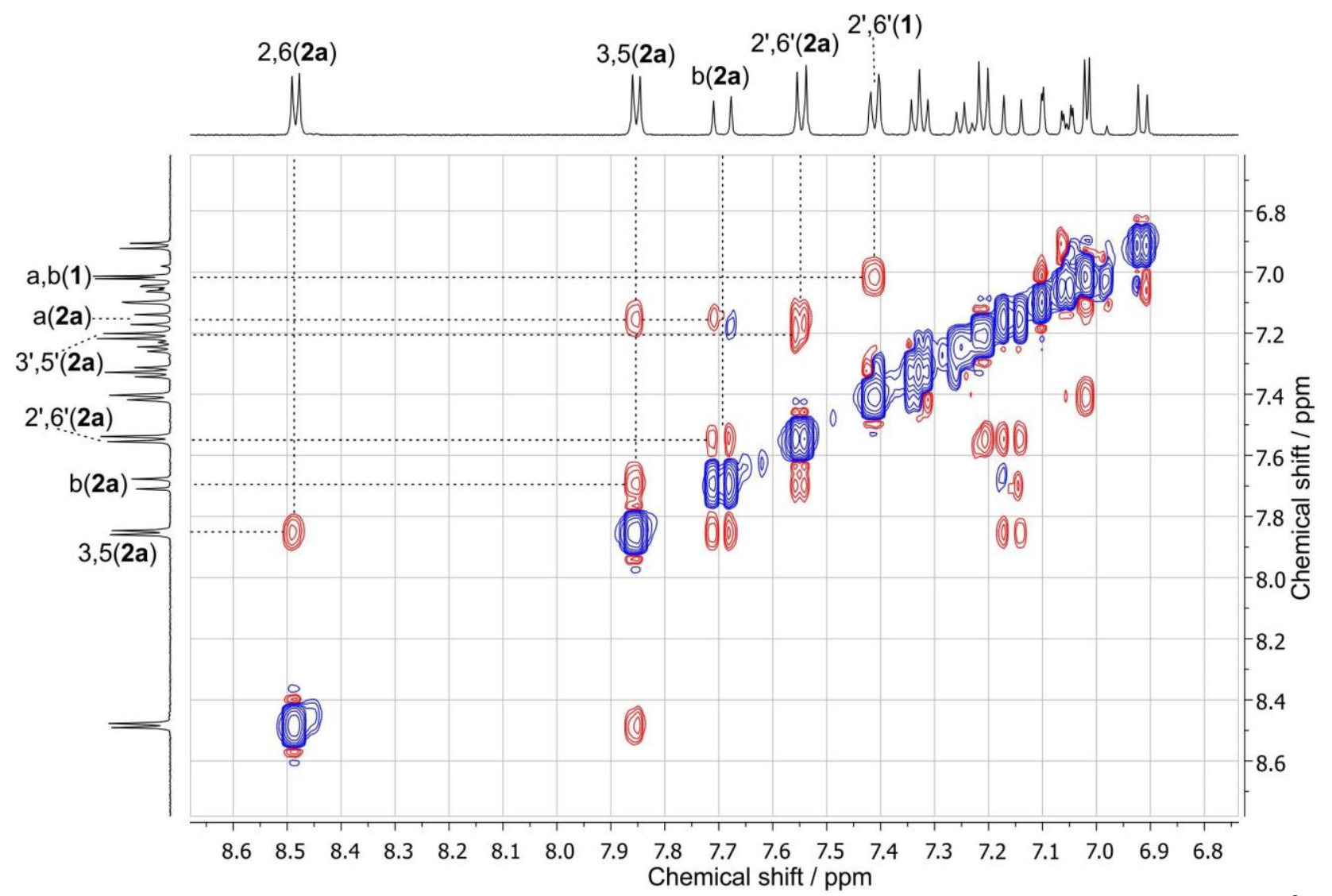

Figure S17. NOESY (296 K, $500 \mathrm{MHz})$ spectrum (aromatic proton region) of equimolar $\left(2 \times 10^{-3} \mathrm{M}\right)$ mixture of $(E)-\mathbf{1}$ and $(E)-\mathbf{2 a}$ in $\mathrm{MeCN}-d_{3}-\mathrm{D}_{2} \mathrm{O}(98: 2, \mathrm{v} / \mathrm{v})$. 


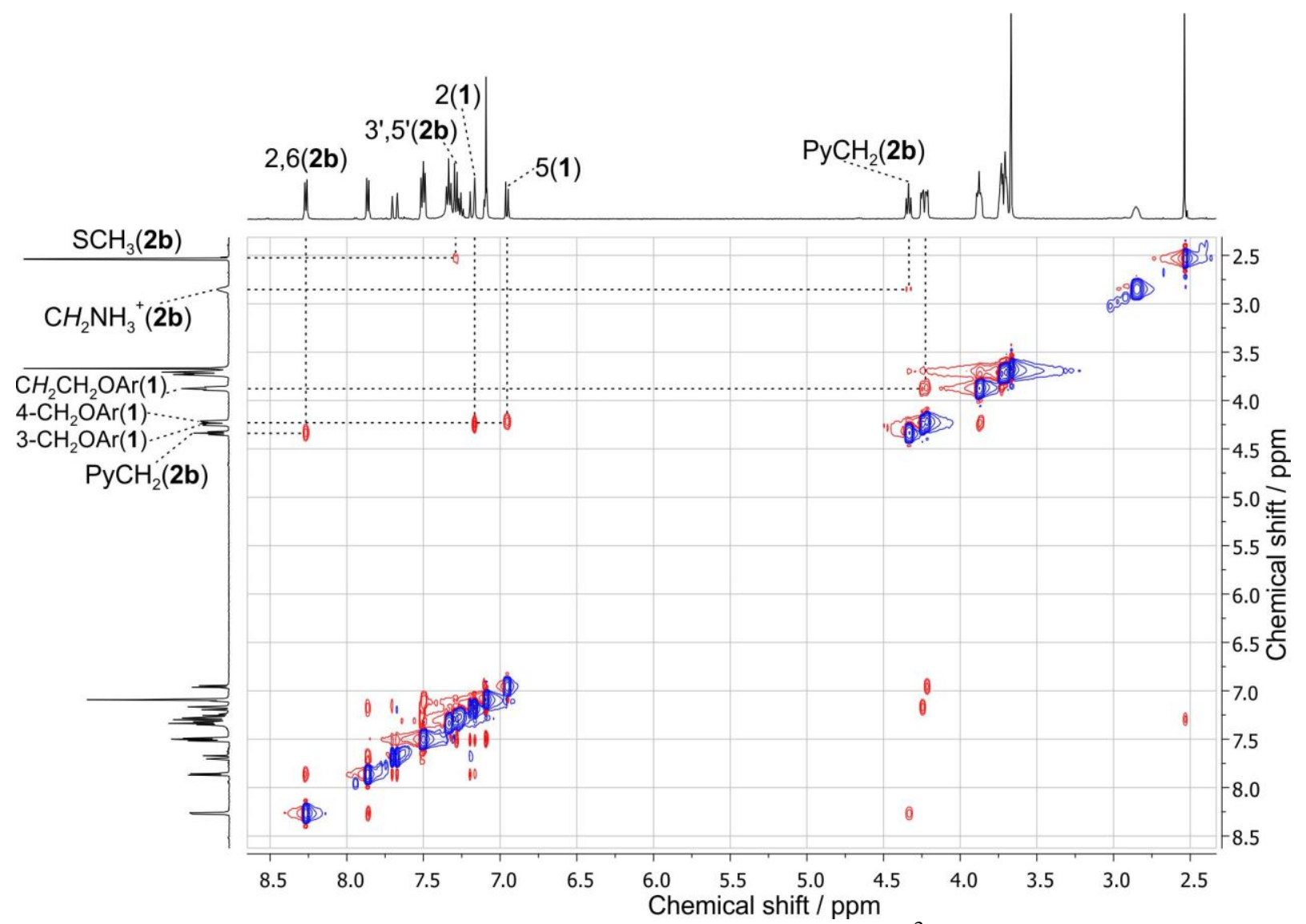

Figure S18. NOESY $(296 \mathrm{~K}, 500 \mathrm{MHz})$ spectrum of equimolar $\left(2 \times 10^{-3} \mathrm{M}\right)$ mixture of $(E)-\mathbf{1}$ and $(E)$ 2b in $\mathrm{MeCN}-d_{3}$.

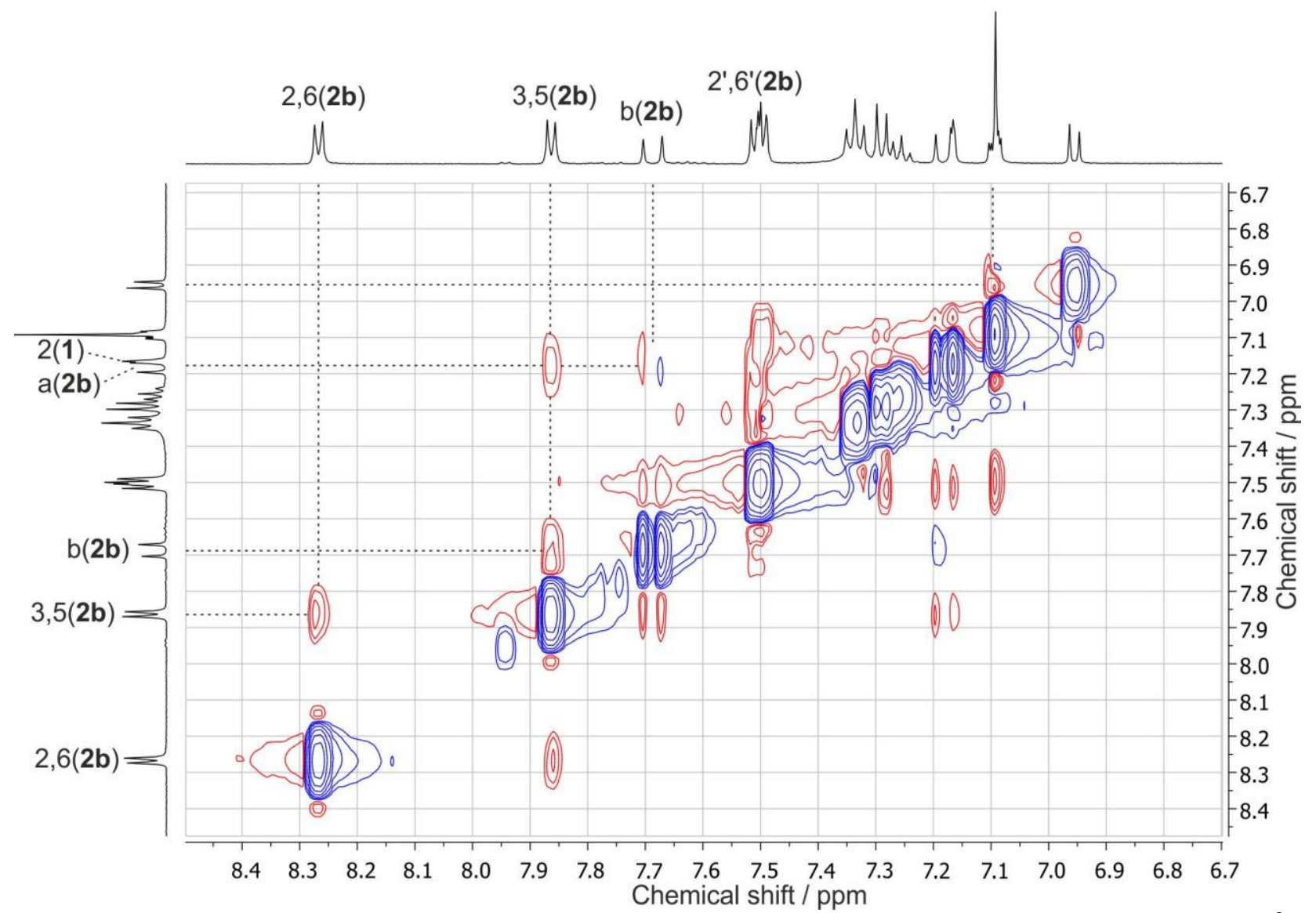

Figure S19. NOESY $(296 \mathrm{~K}, 500 \mathrm{MHz})$ spectrum (aromatic proton region) of equimolar $\left(2 \times 10^{-3} \mathrm{M}\right)$ mixture of $(E)-\mathbf{1}$ and $(E)-\mathbf{2 b}$ in $\mathrm{MeCN}-d_{3}$. 


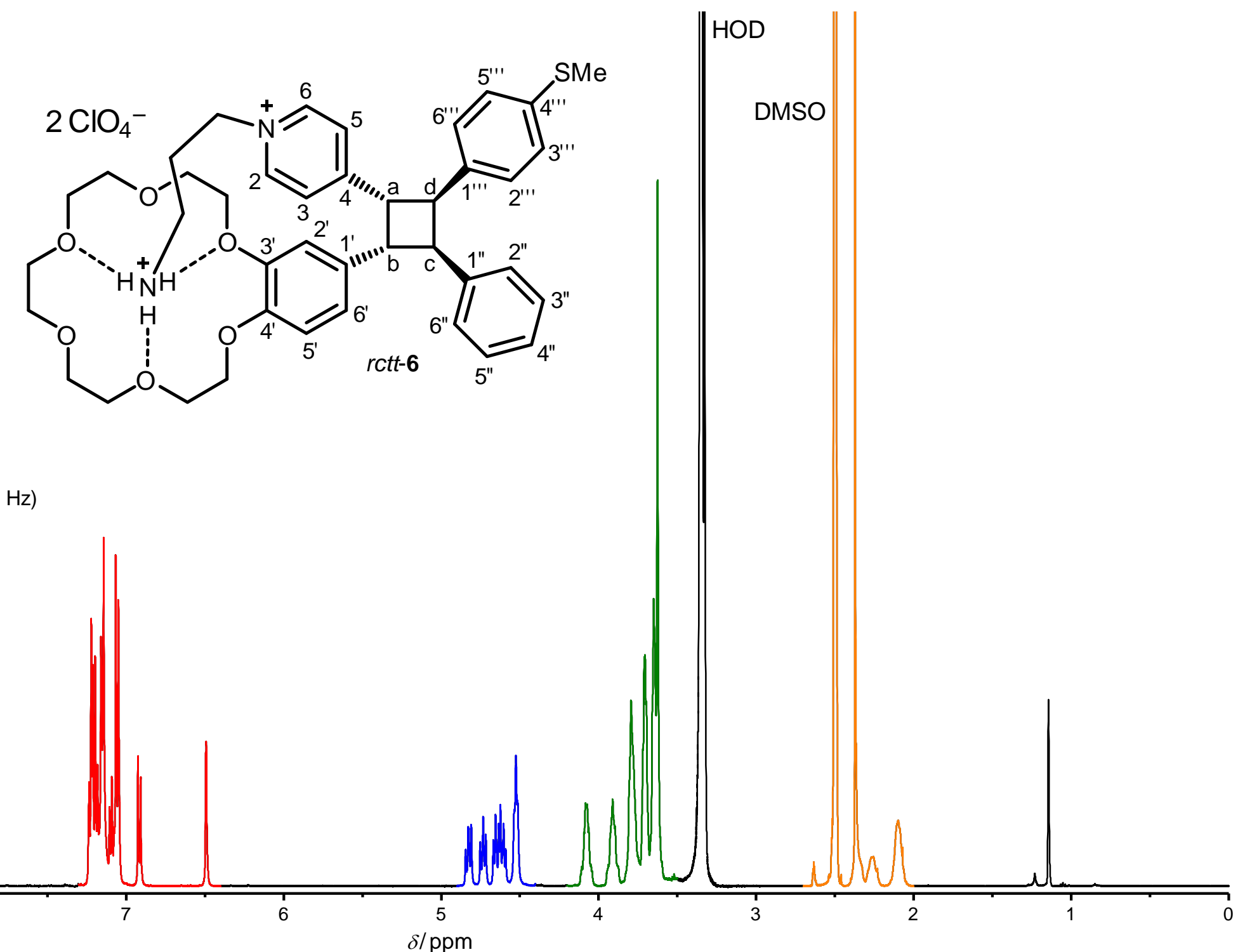

Figure S20. ${ }^{1} \mathrm{H}$ NMR $(296 \mathrm{~K}, 500 \mathrm{MHz})$ spectrum of cyclobutane $r c t t-6$ in DMSO- $d_{6}$. 


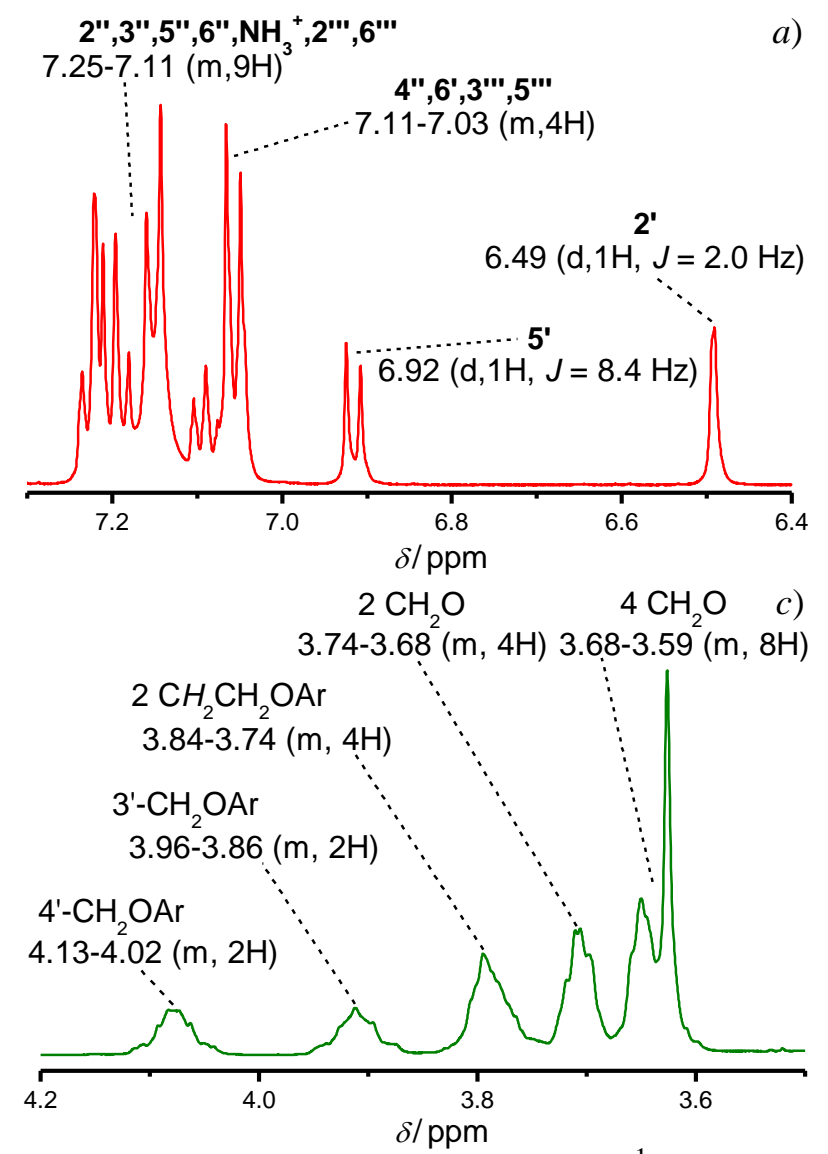

a) $\quad 4.73(\mathrm{dd}, 1 \mathrm{H}, \quad 4.61 \mathrm{ddd}, 1 \mathrm{H}$

b)

$J=9.8$ and $7.6 \mathrm{~Hz}) \quad J=10.2$ and $7.0 \mathrm{~Hz}) \quad \mathrm{PyCH}_{2}$
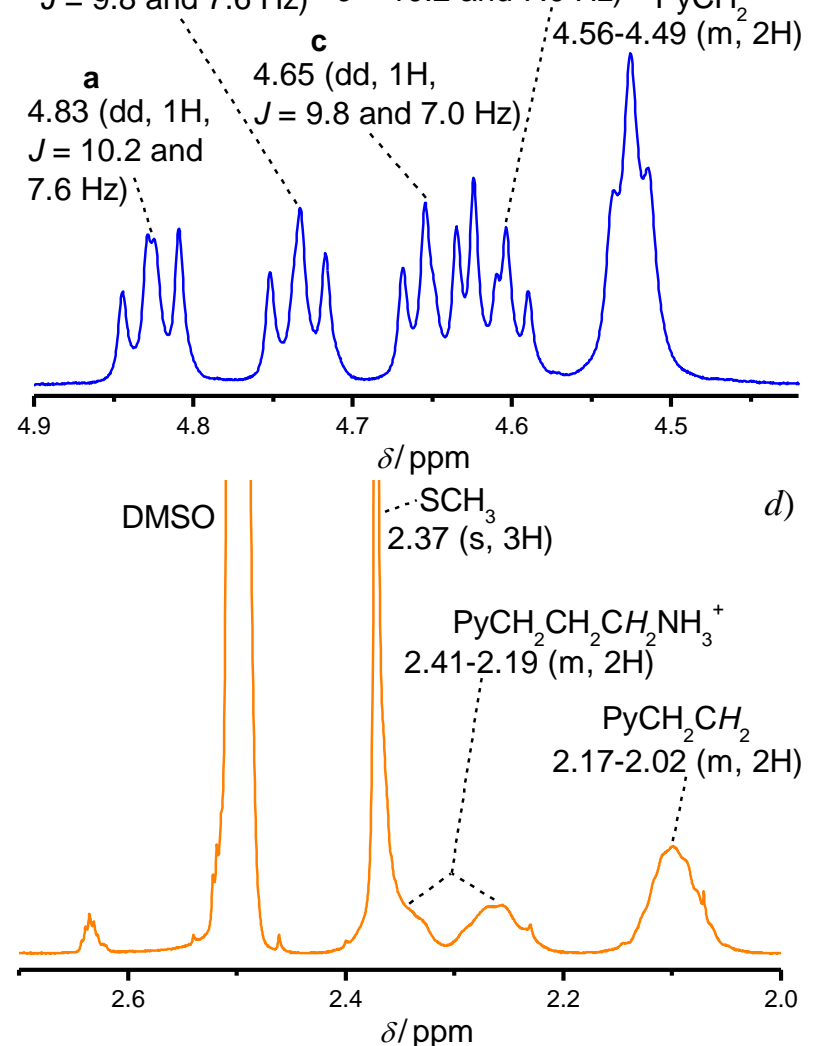

Figure S21. Various fragments of the ${ }^{1} \mathrm{H}$ NMR $(296 \mathrm{~K}, 500 \mathrm{MHz})$ spectrum of cyclobutane $r c t t-6$ in DMSO- $d_{6}$.

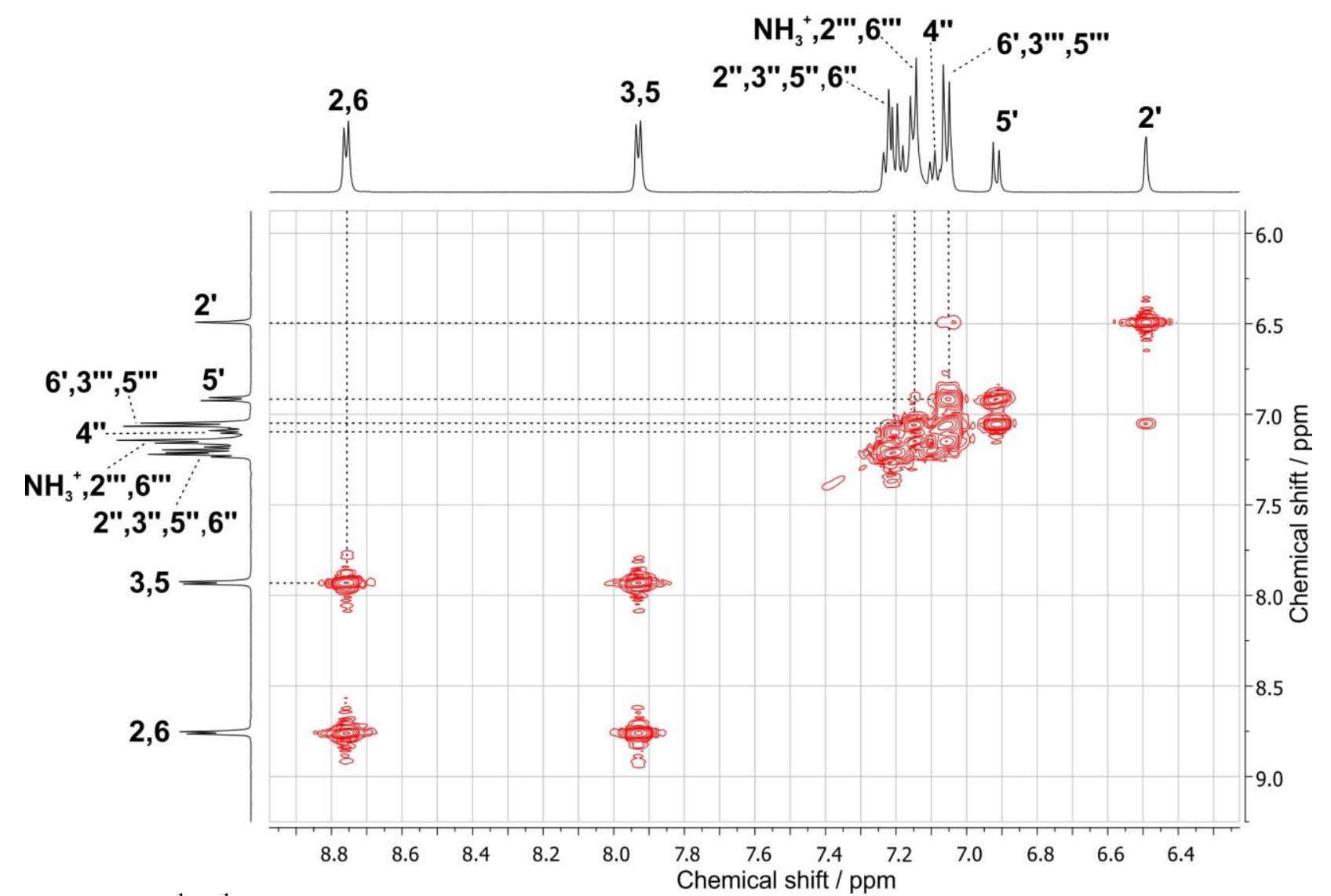

Figure S22. ${ }^{1} \mathrm{H}-{ }^{1} \mathrm{H}$ COSY $(296 \mathrm{~K}, 500 \mathrm{MHz})$ spectrum (aromatic proton region) of cyclobutane derivative $r c t t-6$ in DMSO- $d_{6}$. 


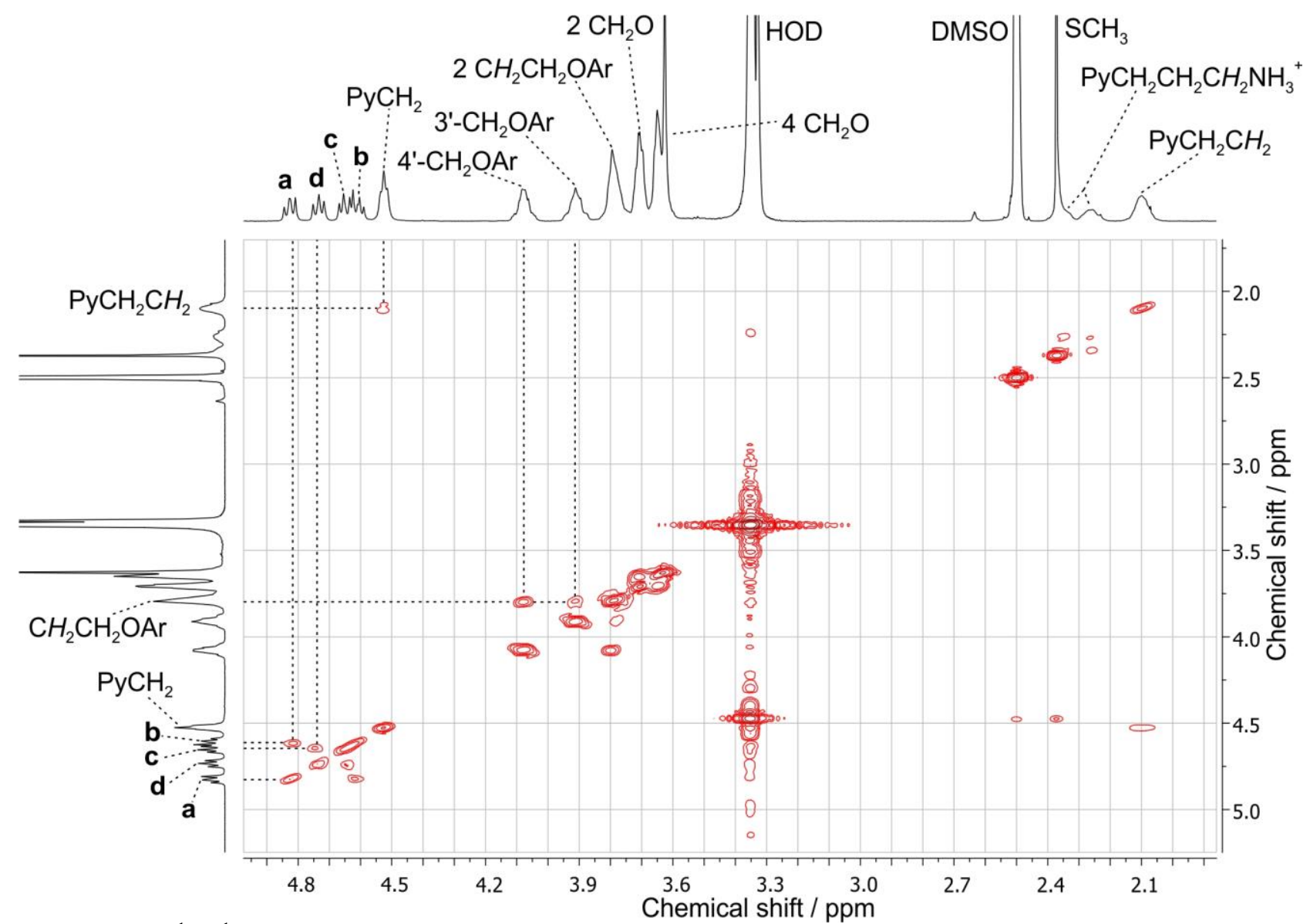

Figure S23. ${ }^{1} \mathrm{H}-{ }^{1} \mathrm{H}$ COSY $(296 \mathrm{~K}, 500 \mathrm{MHz})$ spectrum (aliphatic proton region) of cyclobutane derivative $r c t t-6$ in DMSO- $d_{6}$. 


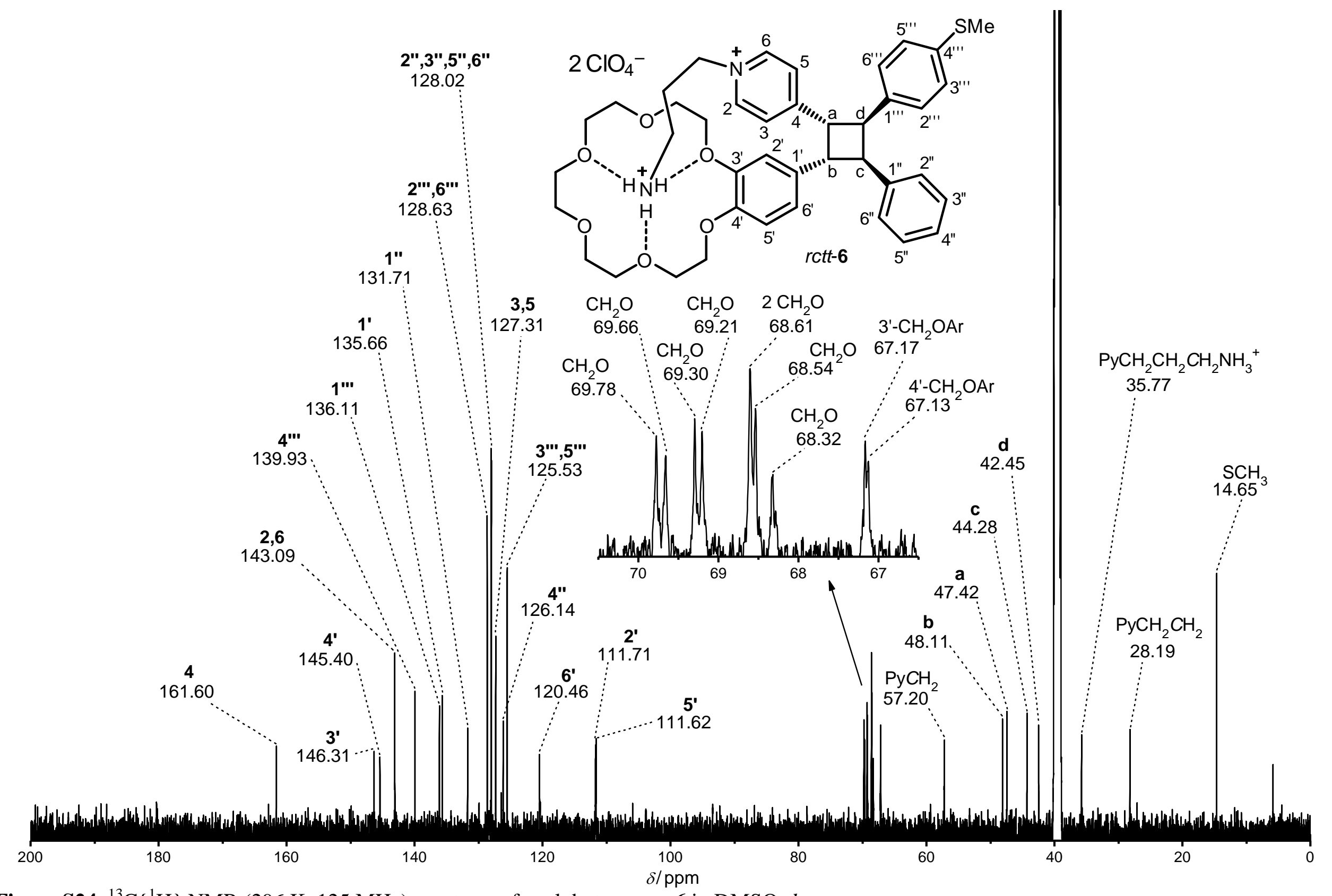

Figure S24. ${ }^{13} \mathrm{C}\left\{{ }^{1} \mathrm{H}\right\}$ NMR $(296 \mathrm{~K}, 125 \mathrm{MHz})$ spectrum of cyclobutane $r c t t-6$ in DMSO- $d_{6}$. 


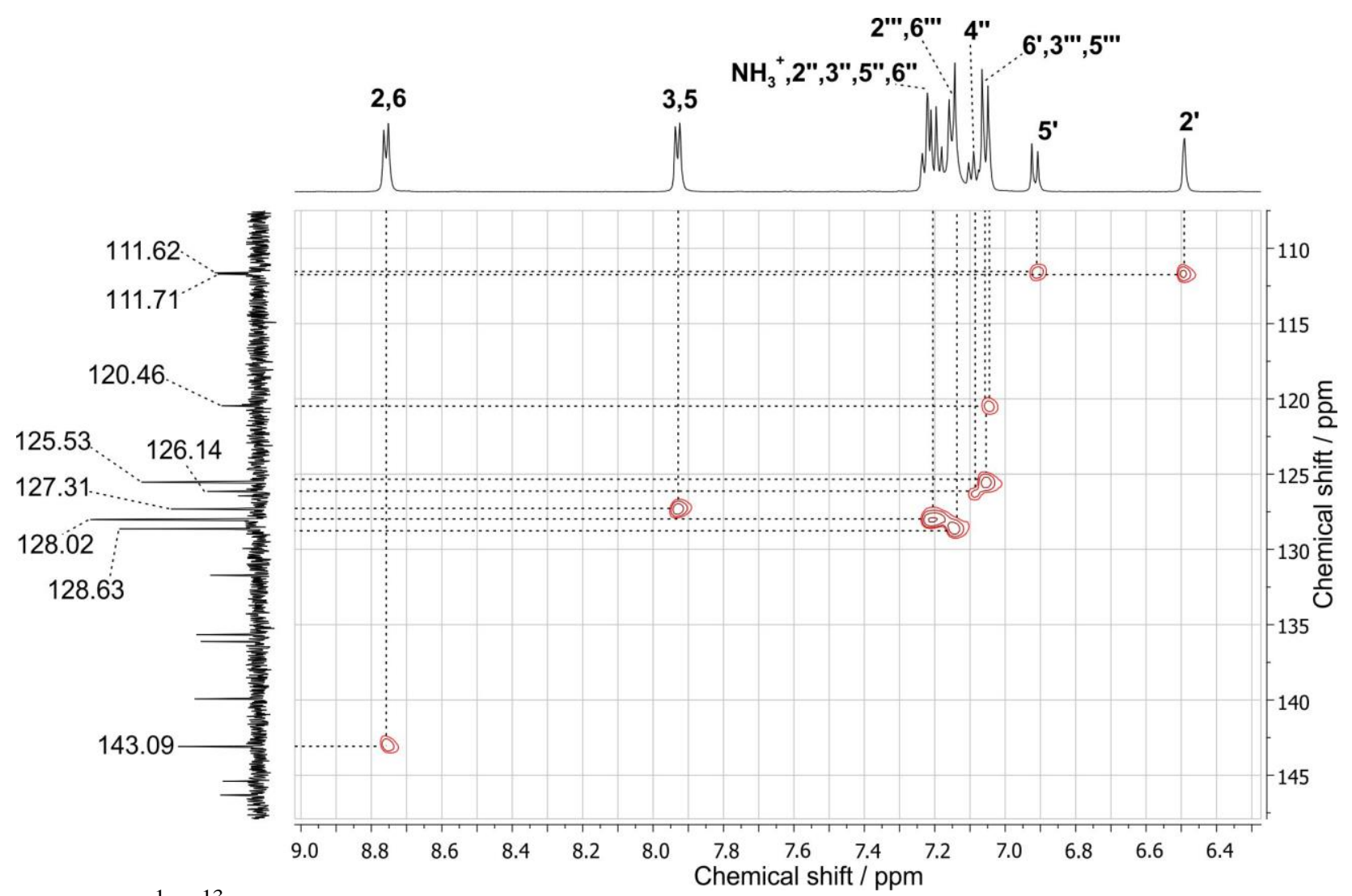

Figure S25. ${ }^{1} \mathrm{H}-{ }^{13} \mathrm{C}$ HSQC $(296 \mathrm{~K}$ ) spectrum (aromatic proton region) of cyclobutane derivative $r c t t-6$ in DMSO- $d_{6}$.

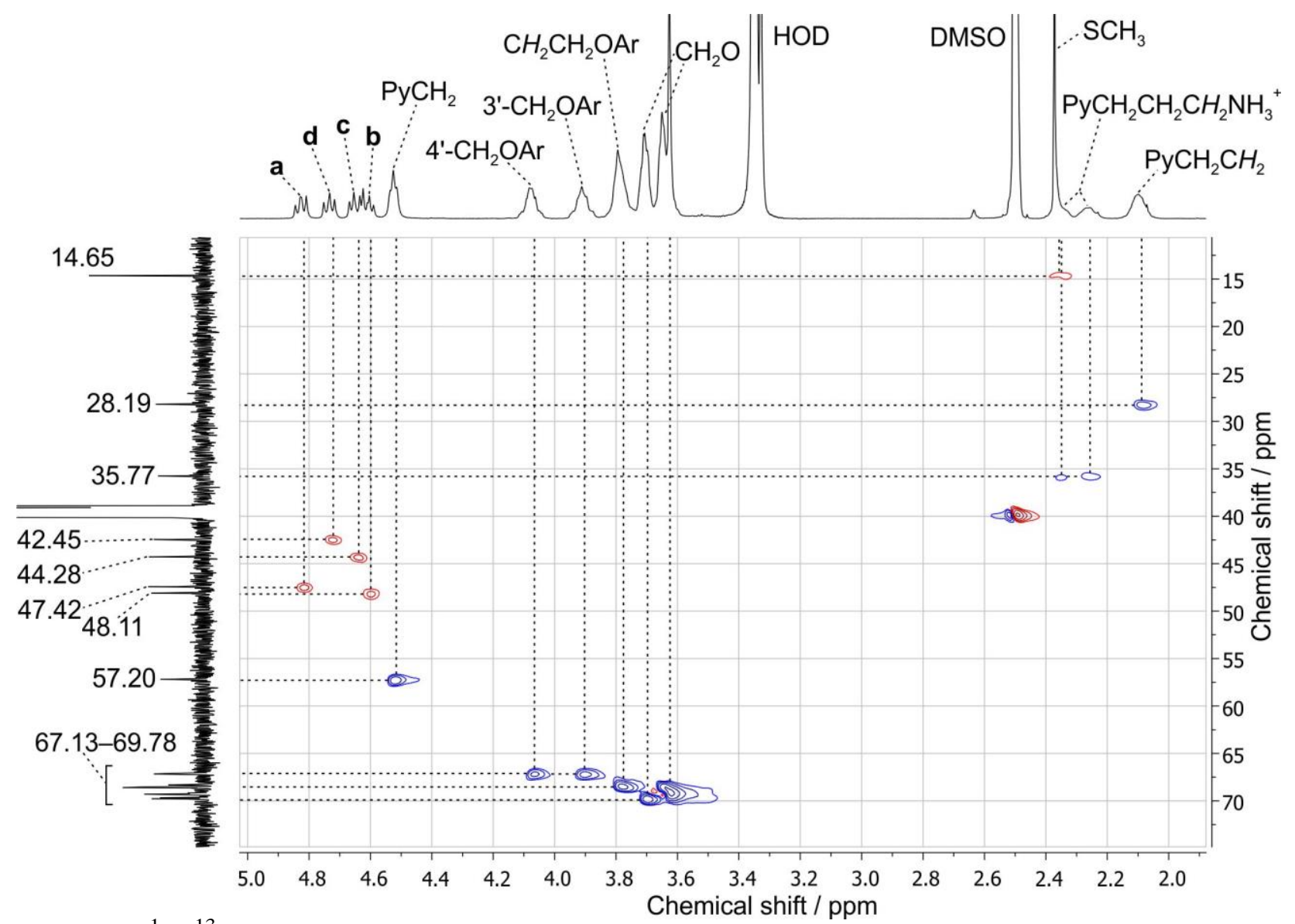

Figure S26. ${ }^{1} \mathrm{H}_{-}{ }^{13} \mathrm{C}$ HSQC $(296 \mathrm{~K})$ spectrum (aliphatic proton region) of cyclobutane derivative $r c t t-6$ in DMSO- $d_{6}$. 
VI Figures S27, S28 and Table S1: X-ray crystallography data

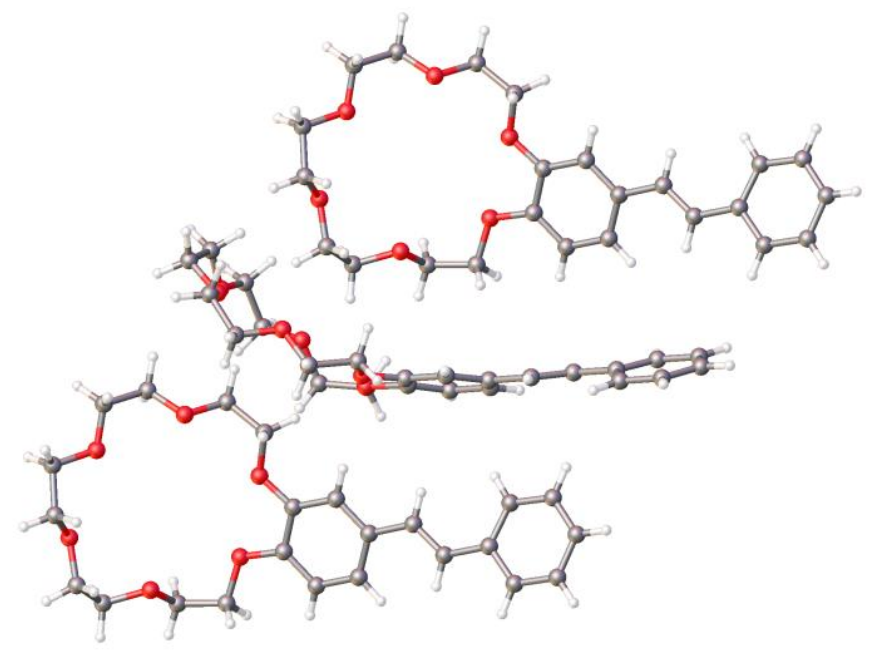

Figure S27. Relative arrangement of stilbene moieties of $(E)-\mathbf{1}$ in the crystal packing.

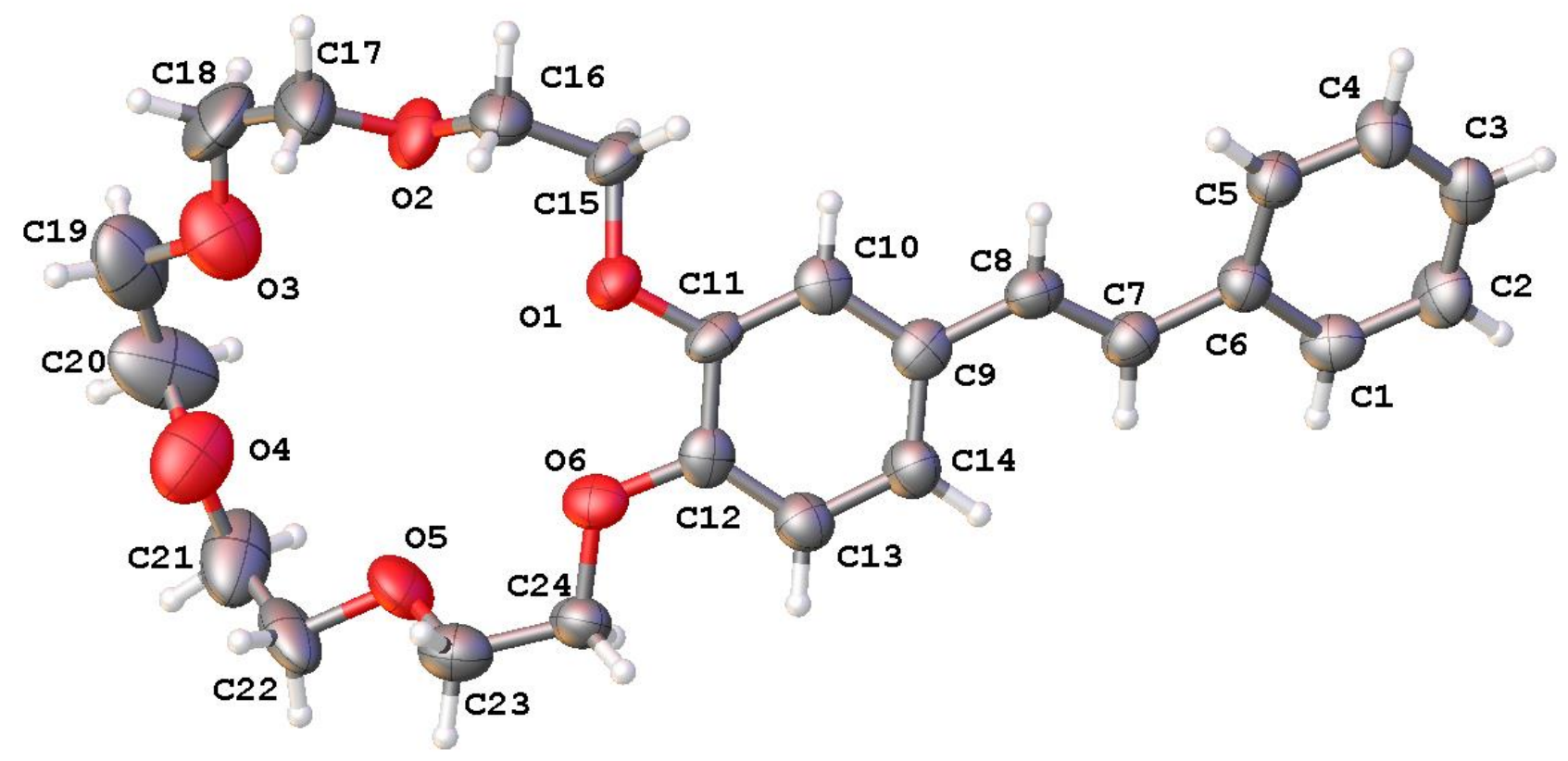

Figure S28. Structure of s-trans conformer of $(E)$-1; thermal displacement ellipsoids are drawn at the $50 \%$ probability level. 
Table S1. Crystal data and structure refinement for $(E)-\mathbf{1}$.

\begin{tabular}{|c|c|}
\hline Formula & $\mathrm{C}_{24} \mathrm{H}_{30} \mathrm{O}_{6}$ \\
\hline Formula weight & 414.49 \\
\hline Temperature, $\mathrm{K}$ & 150 \\
\hline Crystal system & orthorhombic \\
\hline Space group & $P c a 2_{1}$ \\
\hline$a, \AA$ & $8.4527(17)$ \\
\hline$b, \AA$ & $28.734(5)$ \\
\hline$c, \AA$ & $8.8374(19)$ \\
\hline$\alpha, \beta, \gamma^{\circ}$ & $90,90,90$ \\
\hline Volume, $\AA^{3}$ & $2146.4(7)$ \\
\hline$Z$ & 4 \\
\hline$\rho_{\text {calc }}, \mathrm{g} \mathrm{cm}^{-3}$ & 1.273 \\
\hline Radiation & $\operatorname{MoK} \alpha(\lambda=0.71073)$ \\
\hline$\mu, \mathrm{mm}^{-1}$ & 0.091 \\
\hline Crystal size, $\mathrm{mm}^{3}$ & $0.09 \times 0.02 \times 0.01$ \\
\hline $2 \theta$ range for data collection, ${ }^{\circ}$ & 4.26 to 54.00 \\
\hline Index ranges & $-10 \leq h \leq 10,-36 \leq k \leq 35,-11 \leq l \leq 11$ \\
\hline Reflections collected $\mathrm{r}$ & 18331 \\
\hline Independent reflections & $2506\left[R_{\mathrm{int}}=0.2182, R_{\mathrm{sigma}}=0.1517\right]$ \\
\hline Data/restraints/parameters & $2506 / 1 / 272$ \\
\hline Goodness-of-fit on $F^{2}$ & 0.957 \\
\hline Final $\mathrm{R}$ indexes $[I \geq 2 \sigma(I)]$ & $R_{1}=0.0823, w R_{2}=0.1668$ \\
\hline Final $\mathrm{R}$ indexes [all data] & $R_{1}=0.2121, w R_{2}=0.2171$ \\
\hline Largest diff. peak/hole, $\overline{\mathrm{e}} \AA^{-3}$ & $0.25 /-0.24$ \\
\hline Flack parameter & $-1(4)$ \\
\hline
\end{tabular}


VII Figure S29: Notation for conformers of $(E)-\mathbf{1},(E)-\mathbf{2 a}, \mathbf{b}$, and $(E)-\mathbf{1} \cdot(E)-\mathbf{2 a}, \mathbf{b}$

Benzocrown ether moiety of $(E)-1$

(most common conformations)

Stilbene moiety of $(E)-\mathbf{1}$
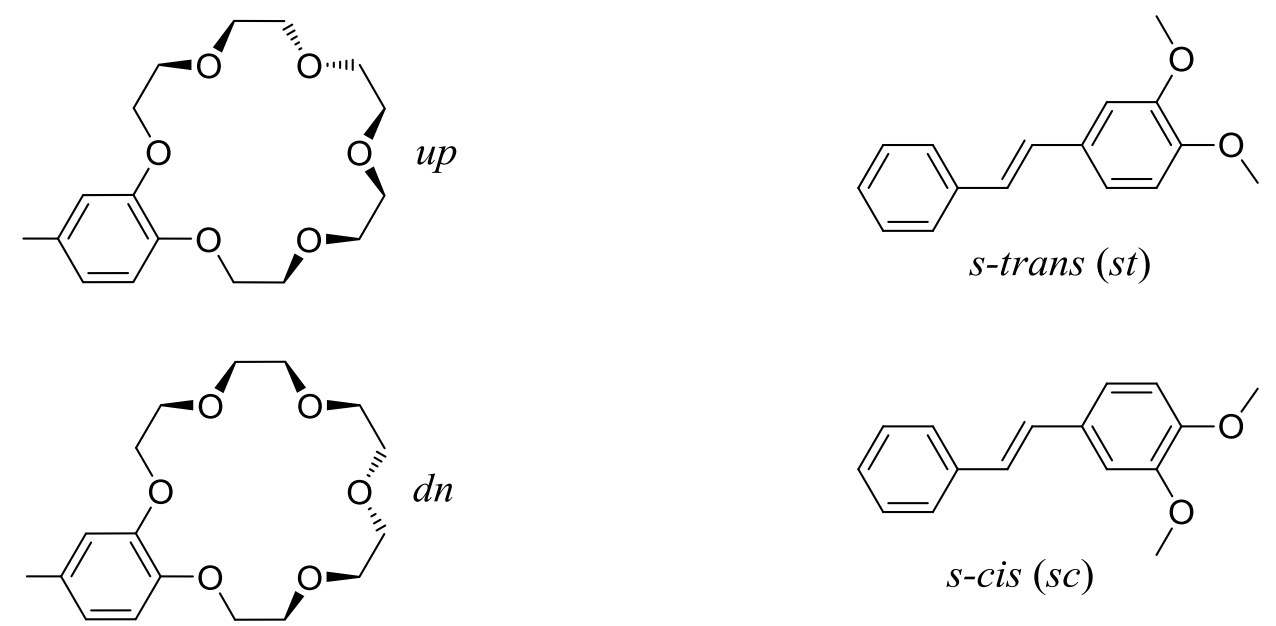

Styrylpyridinium moiety of $(E)-\mathbf{2 a}, \mathbf{b}$

Ammoniopropyl group of (E)-2b

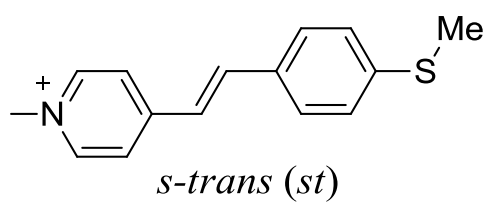

trans,trans $(t t)$

trans, gauche (tg)

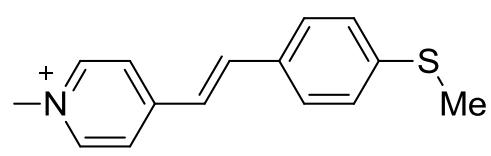

$s-c i s(s c)$

Stacked pseudodimers $(E)-\mathbf{1} \cdot(E)-\mathbf{2 a}, \mathbf{b}$<smiles>COc1ccc(/C=C/c2cc[n+](C)cc2)cc1/C=C/c1ccc(S(C)(=O)=O)cc1</smiles><smiles>COc1ccc(/C=C/c2cc[n+](C)cc2)cc1/C=C/c1ccc(S(C)(C)C)cc1</smiles>

Figure S29. Notation for conformers of compound $(E)-\mathbf{1}$, dyes $(E)-\mathbf{2 a}, \mathbf{b}$, and complexes $(E)-\mathbf{1} \cdot(E)-\mathbf{2 a}, \mathbf{b}$. 
VIII Tables S2-S4, Figure S30: Gibbs free energies in solution for different conformers of $(E)-\mathbf{1},(E)-\mathbf{2 a}, \mathbf{b}$, and $(E)-\mathbf{1} \cdot(E)-\mathbf{2 a}, \mathbf{b}$, as derived from DFT/SMD calculations

Comment: Notation for conformers is shown in Fig. S29.

Table S2. Total and relative Gibbs free energies in MeCN for different conformers of crown compound (E)-1, as derived from DFT/SMD calculations.

\begin{tabular}{lll}
\hline Conformer & $\begin{array}{l}G_{\text {soln }}, \\
\text { Hartrees }\end{array}$ & $\begin{array}{l}\Delta G_{\text {soln }}, \\
\mathrm{kcal} \mathrm{mol}^{-1}\end{array}$ \\
\hline$u p-s c$ & -1383.358960 & 0 \\
$d n-s c$ & -1383.358176 & 0.49 \\
$d n-s t$ & -1383.357395 & 0.98 \\
$u p-s t$ & -1383.356760 & 1.38 \\
\hline
\end{tabular}

Table S3. Total and relative Gibbs free energies in $\mathrm{MeCN}$ for different conformers of dyes $(E)-\mathbf{2 a}$ and $(E)-\mathbf{2 b}$, as derived from DFT/SMD calculations.

\begin{tabular}{llllll}
\hline \multicolumn{2}{c}{$(E)-\mathbf{2 a}$} & & \multicolumn{3}{c}{$(E)-\mathbf{2 b}$} \\
\hline Conformer & $\begin{array}{l}G_{\text {soln }}, \\
\text { Hartrees }\end{array}$ & $\begin{array}{l}\Delta G_{\text {soln }}, \\
\mathrm{kcal} \mathrm{mol}^{-1}\end{array}$ & $\begin{array}{l}\text { Conformer } \\
\text { (see Fig. S29) }\end{array}$ & $\begin{array}{l}G_{\text {soln }}, \\
\text { Hartrees }\end{array}$ & $\begin{array}{l}\Delta G_{\text {soln }}, \\
\mathrm{kcal} \mathrm{mol}^{-1}\end{array}$ \\
\hline gauche-st & -1128.79327751 & 0 & $t t-s t$ & -1168.08111238 & 0 \\
trans - st & -1128.79172503 & 0.97 & $t g-s c$ & -1168.08081918 & 0.18 \\
trans $-s c$ & -1128.79130884 & 1.24 & $t t-s c$ & -1168.07957642 & 0.96 \\
gauche-sc & -1128.78995927 & 2.08 & $t g-s t$ & -1168.07937644 & 1.09 \\
\hline
\end{tabular}


Table S4. Total and relative Gibbs free energies in $\mathrm{MeCN}$ for selected conformers of complexes $(E)-\mathbf{1} \cdot(E)-\mathbf{2 a}$ and $(E)-\mathbf{1} \cdot(E)-\mathbf{2 b}$, as derived from DFT/SMD calculations.

\begin{tabular}{|c|c|c|c|c|c|}
\hline \multicolumn{3}{|c|}{$[(E) \mathbf{- 1} \cdot(E)-\mathbf{2} \mathbf{a}]$} & \multicolumn{3}{|c|}{$[(E)-\mathbf{1} \cdot(E)-\mathbf{2} \mathbf{b}]$} \\
\hline Conformer & $\begin{array}{l}G_{\text {soln }}, \\
\text { Hartrees }\end{array}$ & $\begin{array}{l}\Delta G_{\text {soln }} \\
\mathrm{kcal} \mathrm{mol}^{-1}\end{array}$ & $\begin{array}{l}\text { Conformer } \\
\text { (see Fig. S29) }\end{array}$ & $\begin{array}{l}G_{\text {soln }}, \\
\text { Hartrees }\end{array}$ & $\begin{array}{l}\Delta G_{\text {soln }}, \\
\mathrm{kcal} \mathrm{mol}^{-1}\end{array}$ \\
\hline syn & & & syn & & \\
\hline$(u p-s c) \cdot($ gauche$-s t)$ & -2512.179299 & & $(u p-s c) \cdot(t g-s t)$ & -2551.467311 & 0 \\
\hline$($ up $-s t) \cdot($ gauche$-s c)$ & -2512.177234 & 1.30 & $(u p-s c) \cdot(t t-s t)$ & -2551.463759 & 2.23 \\
\hline$(u p-s c) \cdot($ gauche$-s c)$ & -2512.177455 & 1.15 & $(u p-s t) \cdot(t g-s c)$ & -2551.463933 & 2.12 \\
\hline$(u p-s t) \cdot($ gauche $-s t)$ & -2512.177573 & 1.08 & $(u p-s c) \cdot(t g-s c)$ & -2551.464129 & 2.00 \\
\hline$(d n-s c) \cdot($ gauche $-s t)$ & -2512.177630 & 1.05 & $(u p-s t) \cdot(t g-s t)$ & -2551.463157 & 2.61 \\
\hline$(d n-s t) \cdot($ gauche $-s c)$ & -2512.177535 & 1.11 & $(d n-s c) \cdot(t g-s t)$ & -2551.462776 & 2.85 \\
\hline$(d n-s c) \cdot($ gauche $-s c)$ & -2512.175166 & 2.59 & $(d n-s t) \cdot(t g-s c)$ & -2551.465256 & 1.29 \\
\hline \multirow[t]{2}{*}{$(d n-s t) \cdot($ gauche $-s t)$} & -2512.176028 & 2.05 & $(d n-s c) \cdot(\operatorname{tg}-s c)$ & -2551.462482 & 3.03 \\
\hline & & & $(d n-s t) \cdot(t g-s t)$ & -2551.464758 & 1.60 \\
\hline anti & & & anti & & \\
\hline$(u p-s c) \cdot($ gauche $-s t)$ & -2512.177862 & 0.90 & $(u p-s c) \cdot(t g-s t)$ & -2551.464633 & 1.68 \\
\hline$(u p-s t) \cdot($ gauche$-s c)$ & -2512.179829 & -0.33 & $(u p-s t) \cdot(t g-s c)$ & -2551.463265 & 2.54 \\
\hline$(u p-s c) \cdot($ gauche $-s c)$ & -2512.177308 & 1.25 & $(u p-s c) \cdot(t g-s c)$ & -2551.463607 & 2.32 \\
\hline$(u p-s t) \cdot($ gauche$-s t)$ & -2512.180327 & -0.64 & $(u p-s t) \cdot(t g-s t)$ & -2551.465862 & 0.91 \\
\hline$(d n-s c) \cdot($ gauche $-s t)$ & -2512.175581 & 2.33 & $(d n-s c) \cdot(t g-s t)$ & -2551.461713 & 3.51 \\
\hline$(d n-s t) \cdot($ gauche$-s c)$ & -2512.175632 & 2.30 & $(d n-s t) \cdot(t g-s c)$ & -2551.462482 & 3.03 \\
\hline$(d n-s c) \cdot($ gauche $-s c)$ & -2512.175949 & 2.10 & $(d n-s c) \cdot(\operatorname{tg}-s c)$ & -2551.462889 & 2.77 \\
\hline \multirow[t]{3}{*}{$(d n-s t) \cdot($ gauche $-s t)$} & -2512.177071 & 1.40 & $(d n-s t) \cdot(t g-s t)$ & -2551.465052 & 1.42 \\
\hline & & & unstacked & & \\
\hline & & & $(d n-s t) \cdot(t t-s t)$ & -2551.455375 & 7.49 \\
\hline
\end{tabular}
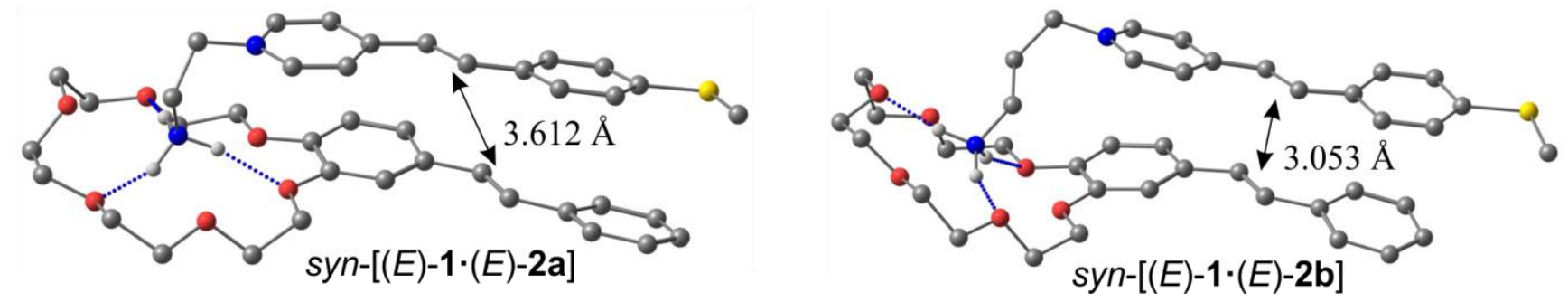

Figure S30. Optimized geometry of the $\mathrm{S}_{1}$ state for most stable conformations of syn-conformers of $(E)-\mathbf{1} \cdot(E)-\mathbf{2 a}$ and $(E)-\mathbf{1} \cdot(E)-\mathbf{2 b}$ in MeCN, according to DFT/SMD calculations; hydrogen atoms are not shown except those of the ammonium group. 


\section{Tables S5-S8: TDDFT calculations}

TDDFT calculations were performed for M06-2X/6-31G(d,p)/SMD $(\mathrm{MeCN})$ geometry.

Table S5. Major molecular orbitals, energies and oscillator strengths of electronic transitions of dyes $(E)-\mathbf{2 a}, \mathbf{b}$ and their pseudodimeric complexes calculated by TDDFT/CAM-B3LYP/6$311 \mathrm{G}(2 \mathrm{~d}, \mathrm{p}) / \mathrm{SMD}(\mathrm{MeCN}){ }^{a}$

\begin{tabular}{|c|c|c|c|c|c|c|c|c|}
\hline \multirow{2}{*}{\multicolumn{2}{|c|}{ Compound }} & \multirow{2}{*}{$\begin{array}{c}E_{\max }{ }^{a b s}, \\
\text { eV }\end{array}$} & \multicolumn{3}{|c|}{$\mathrm{S}_{0}-\mathrm{S}_{1}$} & \multicolumn{3}{|c|}{$\mathrm{S}_{0}-\mathrm{S}_{2}$} \\
\hline & & & $E, \mathrm{eV}$ & $f$ & Orbitals & $E, \mathrm{eV}$ & $f$ & Orbitals \\
\hline \multicolumn{2}{|l|}{$($ gauche$-s t)-(E)-\mathbf{2 a}$} & 3.05 & 3.31 & 1.385 & $\mathrm{H} \rightarrow \mathrm{L}$ & 4.61 & 0.002 & $\mathrm{H}-2 \rightarrow \mathrm{L}$ \\
\hline \multirow[t]{2}{*}{$\operatorname{syn}-[(E)-\mathbf{1} \cdot(E)-\mathbf{2 a}]$} & $(u p-s c) \cdot($ gauche$-s t)$ & \multirow[t]{4}{*}{3.00} & 3.11 & 0.346 & $\underline{\mathrm{H} \rightarrow \mathrm{L}}$ & 3.36 & 0.553 & $\underline{\mathrm{H}-1 \rightarrow \mathrm{L}}$ \\
\hline & $(d n-s c) \cdot($ gauche$-s t)$ & & 3.12 & 0.279 & $\underline{\mathrm{H} \rightarrow \mathrm{L}}$ & 3.36 & 0.571 & $\mathrm{H}-1 \rightarrow \mathrm{L}$ \\
\hline \multirow[t]{2}{*}{ anti- $[(E)-\mathbf{1} \cdot(E)-\mathbf{2 a}]$} & $(u p-s t) \cdot($ gauche$-s t)$ & & 3.05 & 0.241 & $\underline{\mathrm{H} \rightarrow \mathrm{L}}$ & 3.40 & 0.689 & $\mathrm{H}-1 \rightarrow \mathrm{L}$ \\
\hline & $(u p-s t) \cdot($ gauche$-s c)$ & & 3.13 & 0.208 & $\underline{\mathrm{H} \rightarrow \mathrm{L}}$ & 3.32 & 0.704 & $\mathrm{H}-1 \rightarrow \mathrm{L}$ \\
\hline \multicolumn{2}{|l|}{$(t t-s t)-(E)-\mathbf{2 b}$} & 3.09 & 3.37 & 1.410 & $\mathrm{H} \rightarrow \mathrm{L}$ & 4.63 & 0.002 & $\mathrm{H} \rightarrow \mathrm{L}+3$ \\
\hline \multirow{8}{*}{$\operatorname{syn}-[(E)-\mathbf{1} \cdot(E)-\mathbf{2 b}]$} & $(u p-s c) \cdot(t g-s t)$ & \multirow[t]{10}{*}{3.08} & 3.31 & 0.847 & $\mathrm{H}-1 \rightarrow \mathrm{L}$ & 3.34 & 0.133 & $\underline{\mathrm{H} \rightarrow \mathrm{L}}$ \\
\hline & $(d n-s t) \cdot(t g-s c)$ & & 3.29 & 0.587 & $\underline{\mathrm{H} \rightarrow \mathrm{L}}$ & 3.31 & 0.335 & $\mathrm{H}-1 \rightarrow \mathrm{L}$ \\
\hline & $(d n-s t) \cdot(t g-s t)$ & & 3.32 & 0.925 & $\mathrm{H}-1 \rightarrow \mathrm{L}$ & 3.39 & 0.022 & $\underline{\mathrm{H} \rightarrow \mathrm{L}}$ \\
\hline & $(u p-s c) \cdot(t g-s c)$ & & 3.25 & 0.518 & $\underline{\mathrm{H} \rightarrow \mathrm{L}}$ & 3.39 & 0.352 & $\underline{\mathrm{H} \rightarrow \mathrm{L}^{b}}$ \\
\hline & $(u p-s t) \cdot(t g-s c)$ & & 3.28 & 0.265 & $\underline{\mathrm{H} \rightarrow \mathrm{L}}$ & 3.30 & 0.664 & $\underline{\mathrm{H} \rightarrow \mathrm{L}^{b}}$ \\
\hline & $(u p-s t) \cdot(t g-s t)$ & & 3.30 & 0.880 & $\mathrm{H}-1 \rightarrow \mathrm{L}$ & 3.38 & 0.068 & $\underline{\mathrm{H} \rightarrow \mathrm{L}}$ \\
\hline & $(d n-s c) \cdot(t g-s t)$ & & 3.30 & 0.771 & $\mathrm{H}-1 \rightarrow \mathrm{L}$ & 3.36 & 0.206 & $\underline{\mathrm{H} \rightarrow \mathrm{L}}$ \\
\hline & $(d n-s c) \cdot(\operatorname{tg}-s c)$ & & 3.27 & 0.611 & $\mathrm{H}-1 \rightarrow \mathrm{L}$ & 3.38 & 0.253 & $\underline{\mathrm{H} \rightarrow \mathrm{L}}$ \\
\hline \multirow[t]{2}{*}{ anti- $[(E)-\mathbf{1} \cdot(E)-\mathbf{2} \mathbf{b}]$} & $(u p-s t) \cdot(t g-s t)$ & & 3.28 & 0.281 & $\underline{\mathrm{H} \rightarrow \mathrm{L}}$ & 3.34 & 0.663 & $\mathrm{H}-1 \rightarrow \mathrm{L}$ \\
\hline & $(d n-s t) \cdot(t g-s t)$ & & 3.28 & 0.364 & $\underline{\mathrm{H} \rightarrow \mathrm{L}}$ & 3.35 & 0.588 & $\mathrm{H}-1 \rightarrow \mathrm{L}$ \\
\hline syn- $[(E)-\mathbf{3} \cdot(E)-\mathbf{2 b}]$ & $(u p-s t) \cdot(t g-s c)$ & \multirow[t]{2}{*}{3.08} & 3.30 & 0.889 & $\mathrm{H} \rightarrow \mathrm{L}$ & 3.45 & 0.033 & $\mathrm{H}-1 \rightarrow \mathrm{L}$ \\
\hline anti- $[(E)-\mathbf{3} \cdot(E)-\mathbf{2} \mathbf{b}]$ & $(u p-s c) \cdot(t g-s c)$ & & 3.29 & 0.959 & $\mathrm{H} \rightarrow \mathrm{L}$ & 3.52 & 0.006 & $\underline{\mathrm{H}-1 \rightarrow \mathrm{L}}$ \\
\hline
\end{tabular}

${ }^{a} E_{\max }{ }^{\text {abs }}$ is the energy (eV) corresponding to the long-wavelength absortion maximum of the compound in MeCN; $\mathrm{H}$ is HOMO, L is LUMO, the underlined transitions correspond to the donor (stilbene) to acceptor (dye) charge transfer.

${ }^{b}$ The contribution of $\mathrm{H}-1 \rightarrow \mathrm{L}$ was slightly less than half. 
Table S6. Spatial distributions of the molecular orbitals of the pseudodimeric complexes calculated by TDDFT/CAM-B3LYP/6-311G(2d,p)/SMD(MeCN).

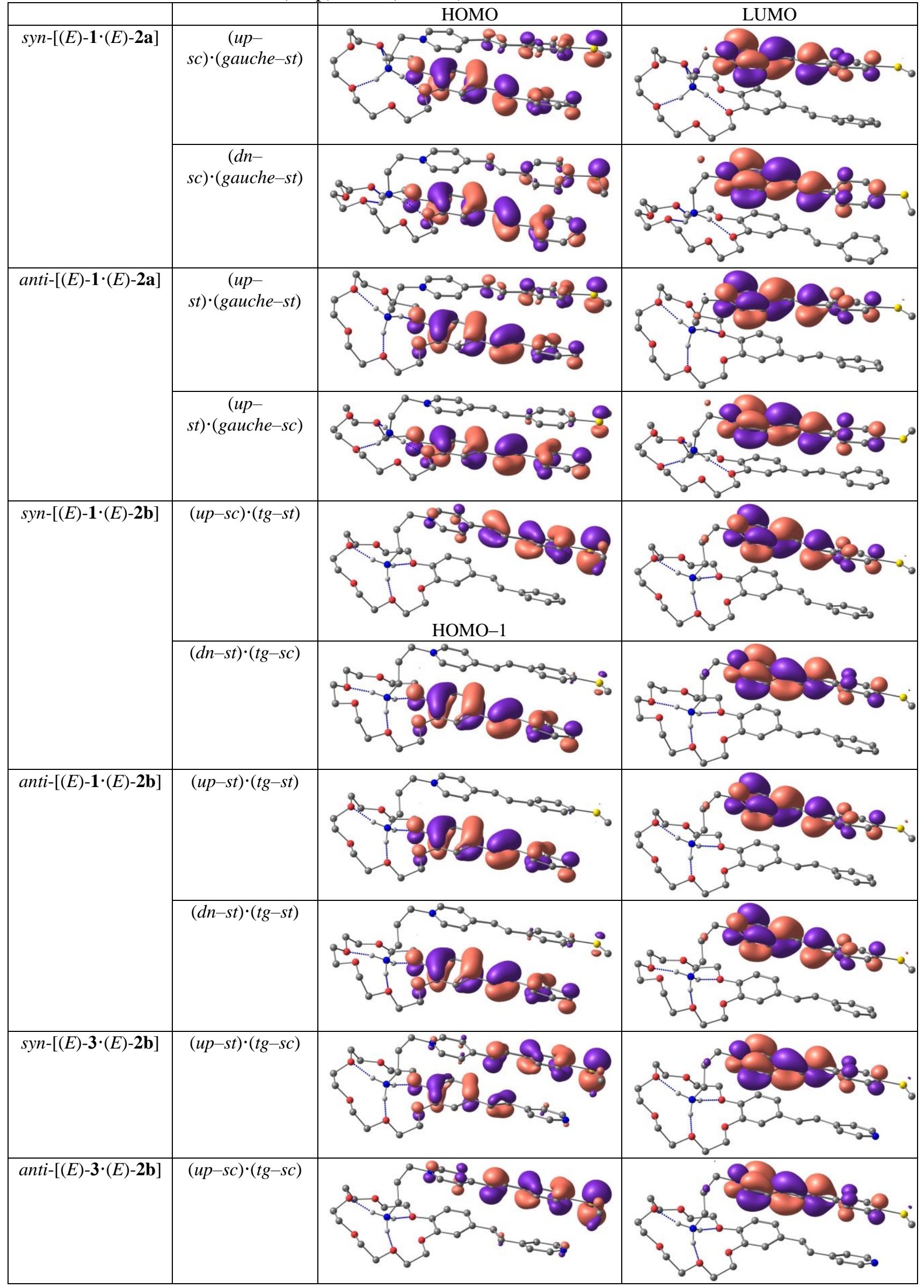


Table S7. Major molecular orbitals, energies and oscillator strengths of electronic transitions of dyes $(E)-\mathbf{2 a}, \mathbf{b}$ and their pseudodimeric complexes calculated by TDDFT/M06-2X/6$311 \mathrm{G}(2 \mathrm{~d}, \mathrm{p}) / \mathrm{SMD}(\mathrm{MeCN}){ }^{a}$

\begin{tabular}{|c|c|c|c|c|c|c|c|c|}
\hline \multirow{2}{*}{\multicolumn{2}{|c|}{ Compound }} & \multirow{2}{*}{$\begin{array}{c}E_{\max }{ }^{\text {abs }}, \\
\text { eV } \\
\end{array}$} & \multicolumn{3}{|c|}{$\mathrm{S}_{0}-\mathrm{S}_{1}$} & \multicolumn{3}{|c|}{$\mathrm{S}_{0}-\mathrm{S}_{2}$} \\
\hline & & & $E, \mathrm{eV}$ & $f$ & Orbitals & $E, \mathrm{eV}$ & $f$ & Orbitals \\
\hline \multicolumn{2}{|l|}{$($ gauche$-s t)-(E)-\mathbf{2 a}$} & 3.05 & 3.27 & 1.354 & $\mathrm{H} \rightarrow \mathrm{L}$ & 4.62 & 0.003 & $\mathrm{H}-2 \rightarrow \mathrm{L}$ \\
\hline \multirow[t]{2}{*}{$\operatorname{syn}-[(E)-\mathbf{1} \cdot(E)-\mathbf{2 a}]$} & $(u p-s c) \cdot($ gauche$-s t)$ & \multirow[t]{4}{*}{3.00} & 3.09 & 0.409 & $\underline{\mathrm{H} \rightarrow \mathrm{L}}$ & 3.33 & 0.497 & $\mathrm{H}-1 \rightarrow \mathrm{L}$ \\
\hline & $(d n-s c) \cdot($ gauche $-s t)$ & & 3.11 & 0.340 & $\underline{\mathrm{H} \rightarrow \mathrm{L}}$ & 3.33 & 0.523 & $\mathrm{H}-1 \rightarrow \mathrm{L}$ \\
\hline \multirow[t]{2}{*}{$\operatorname{anti}-[(E)-\mathbf{1} \cdot(E)-\mathbf{2 a}]$} & $(u p-s t) \cdot($ gauche$-s t)$ & & 3.02 & 0.271 & $\mathrm{H} \rightarrow \mathrm{L}$ & 3.36 & 0.666 & $\mathrm{H}-1 \rightarrow \mathrm{L}$ \\
\hline & $(u p-s t) \cdot($ gauche$-s c)$ & & 3.11 & 0.264 & $\mathrm{H} \rightarrow \mathrm{L}$ & 3.30 & 0.654 & $\mathrm{H}-1 \rightarrow \mathrm{L}$ \\
\hline \multicolumn{2}{|l|}{$(t t-s t)-(E)-2 \mathbf{b}$} & 3.09 & 3.08 & 1.382 & $\mathrm{H} \rightarrow \mathrm{L}$ & 4.65 & 0.003 & $\mathrm{H}-2 \rightarrow \mathrm{L}$ \\
\hline \multirow[t]{2}{*}{$\operatorname{syn}-[(E)-\mathbf{1} \cdot(E)-\mathbf{2 b}]$} & $(u p-s c) \cdot(t g-s t)$ & \multirow[t]{4}{*}{3.08} & 3.27 & 0.923 & $\mathrm{H}-1 \rightarrow \mathrm{L}$ & 3.33 & 0.060 & $\underline{\mathrm{H} \rightarrow \mathrm{L}}$ \\
\hline & $(d n-s t) \cdot(t g-s c)$ & & 3.25 & 0.763 & $\underline{\mathrm{H} \rightarrow \mathrm{L}}$ & 3.28 & 0.160 & $\mathrm{H}-1 \rightarrow \mathrm{L}$ \\
\hline \multirow[t]{2}{*}{ anti- $[(E)-\mathbf{1} \cdot(E)-\mathbf{2 b}]$} & $(u p-s t) \cdot(t g-s t)$ & & 3.25 & 0.440 & $\mathrm{H} \rightarrow \mathrm{L}$ & 3.31 & 0.513 & $\mathrm{H}-1 \rightarrow \mathrm{L}$ \\
\hline & $(d n-s t) \cdot(t g-s t)$ & & 3.24 & 0.479 & $\mathrm{H} \rightarrow \mathrm{L}$ & 3.33 & 0.480 & $\mathrm{H}-1 \rightarrow \mathrm{L}$ \\
\hline $\operatorname{syn}-[(E)-\mathbf{3} \cdot(E)-\mathbf{2 b}]$ & $(u p-s t) \cdot(t g-s c)$ & \multirow[t]{2}{*}{3.08} & 3.25 & 0.900 & $\mathrm{H} \rightarrow \mathrm{L}$ & 3.42 & 0.028 & $\mathrm{H}-1 \rightarrow \mathrm{L}$ \\
\hline anti- $[(E)-\mathbf{3} \cdot(E)-\mathbf{2 b}]$ & $(u p-s c) \cdot(\operatorname{tg}-s c)$ & & 3.25 & 0.957 & $\mathrm{H} \rightarrow \mathrm{L}$ & 3.49 & 0.008 & $\overline{\mathrm{H}-1 \rightarrow \mathrm{L}}$ \\
\hline
\end{tabular}

${ }^{a} E_{\max }{ }^{\text {abs }}$ is the energy $(\mathrm{eV})$ corresponding to the long-wavelength absortion maximum of the compound in $\mathrm{MeCN}$; $\mathrm{H}$ is HOMO, L is LUMO, the underlined transitions correspond to the donor (stilbene) to acceptor (dye) charge transfer. 
Table S8. Spatial distributions of the molecular orbitals of the pseudodimeric complexes calculated by TDDFT/M06-2X/6-311G(2d,p)/SMD(MeCN).

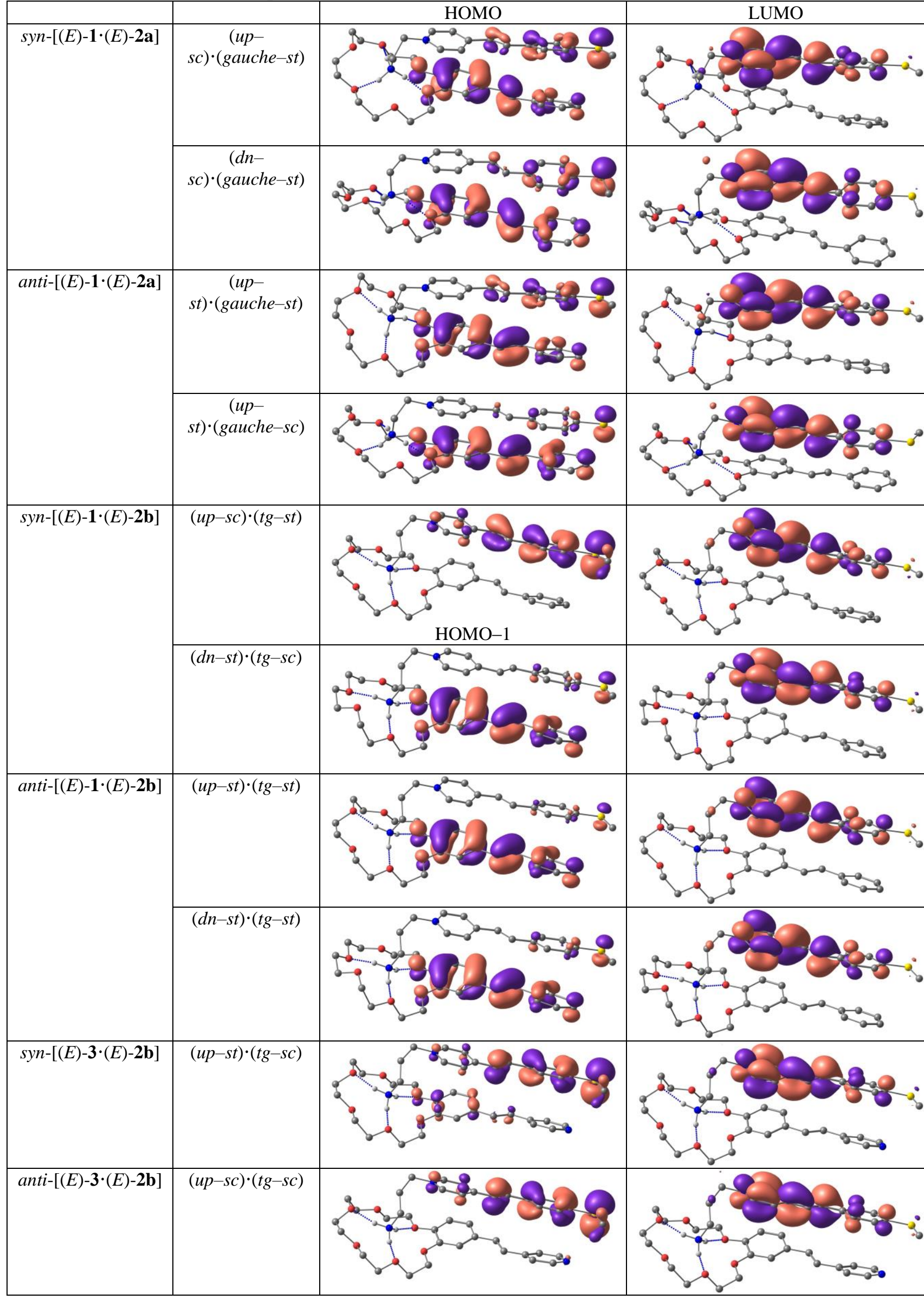


X Figures S31-S37: NMR and ESI mass spectra of synthesized compounds

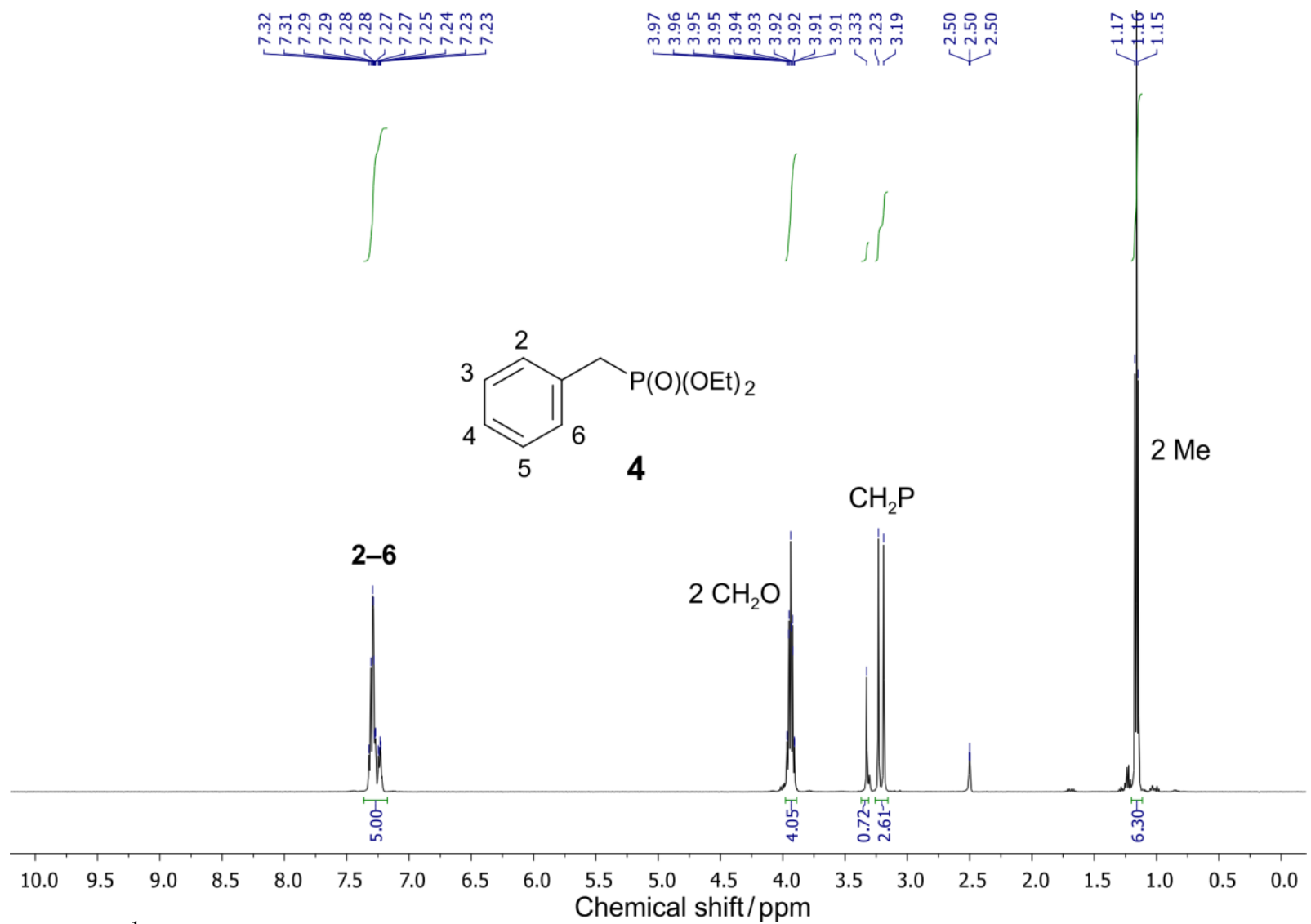

Figure S31. ${ }^{1} \mathrm{H}$ NMR (296 K, $500 \mathrm{MHz}$ ) spectrum of diethyl benzylphosphonate (4) in DMSO- $d_{6}$.

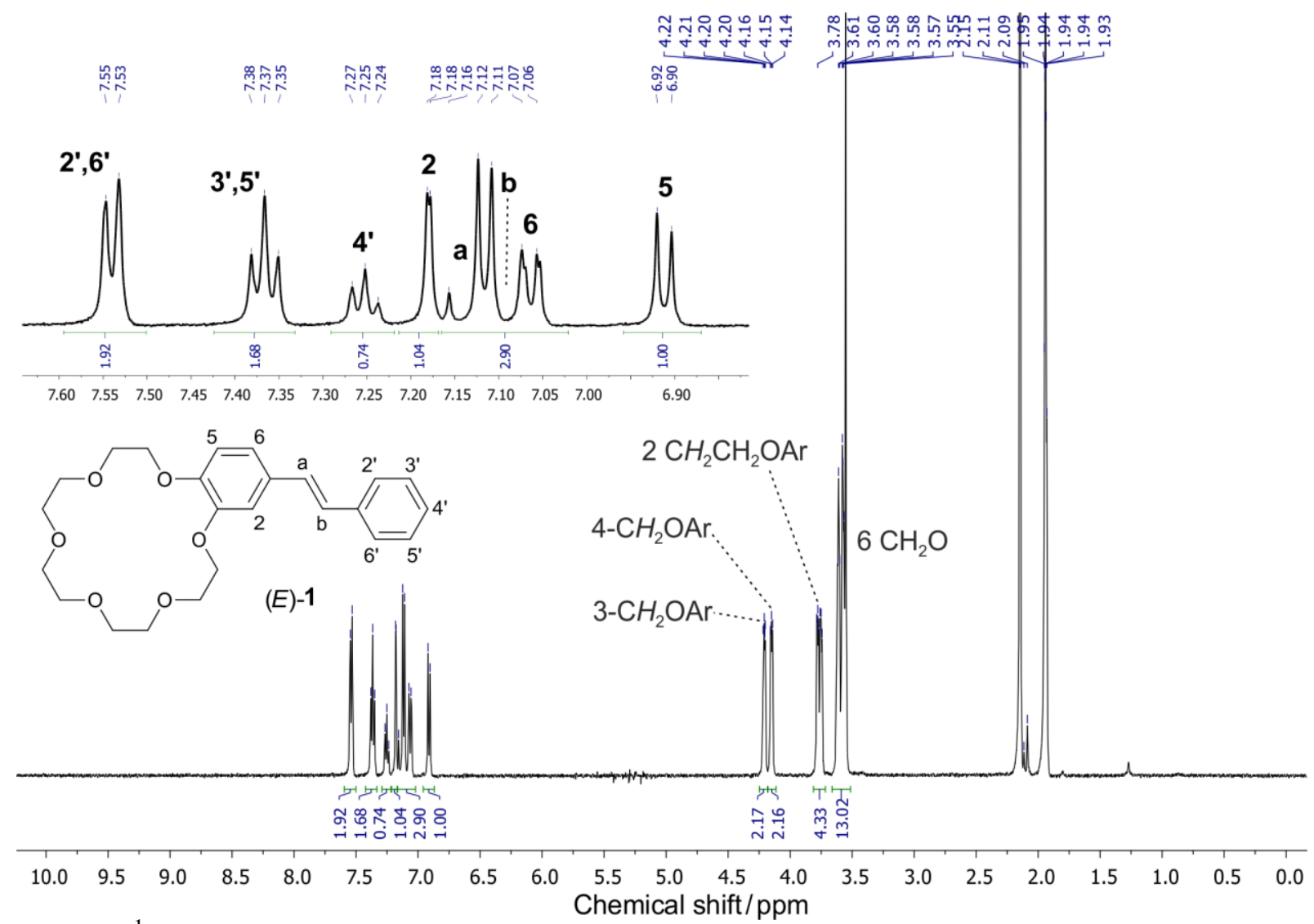

Figure S32. ${ }^{1} \mathrm{H}$ NMR $(296 \mathrm{~K}, 500 \mathrm{MHz})$ spectrum of (18-crown-6)stilbene $(E)-\mathbf{1}$ in MeCN- $d_{3}$. 


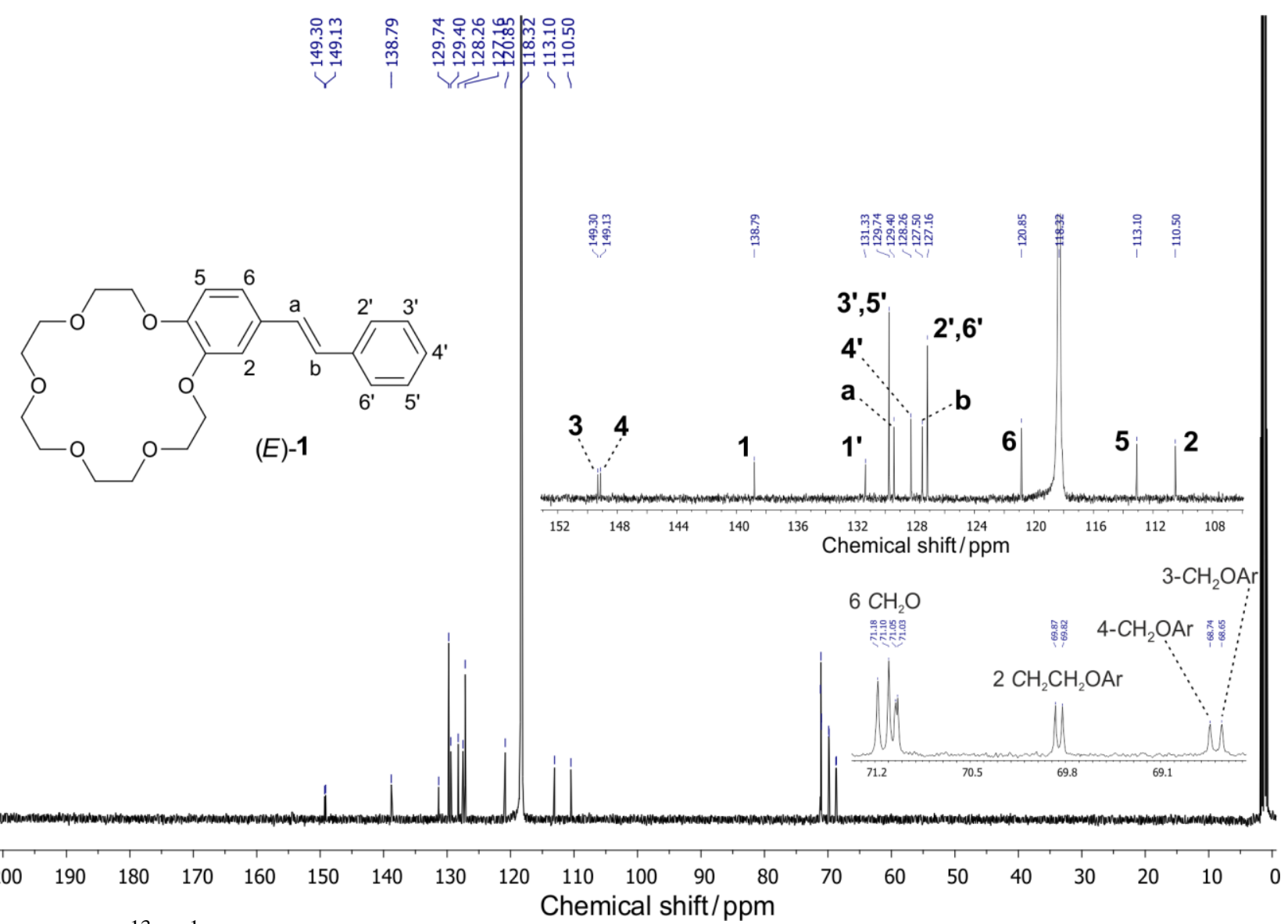

Figure S33. ${ }^{13} \mathrm{C}\left\{{ }^{1} \mathrm{H}\right\}$ NMR $(296 \mathrm{~K}, 125 \mathrm{MHz})$ spectrum of (18-crown-6)stilbene $(E)-\mathbf{1}$ in MeCN-d $d_{3}$.
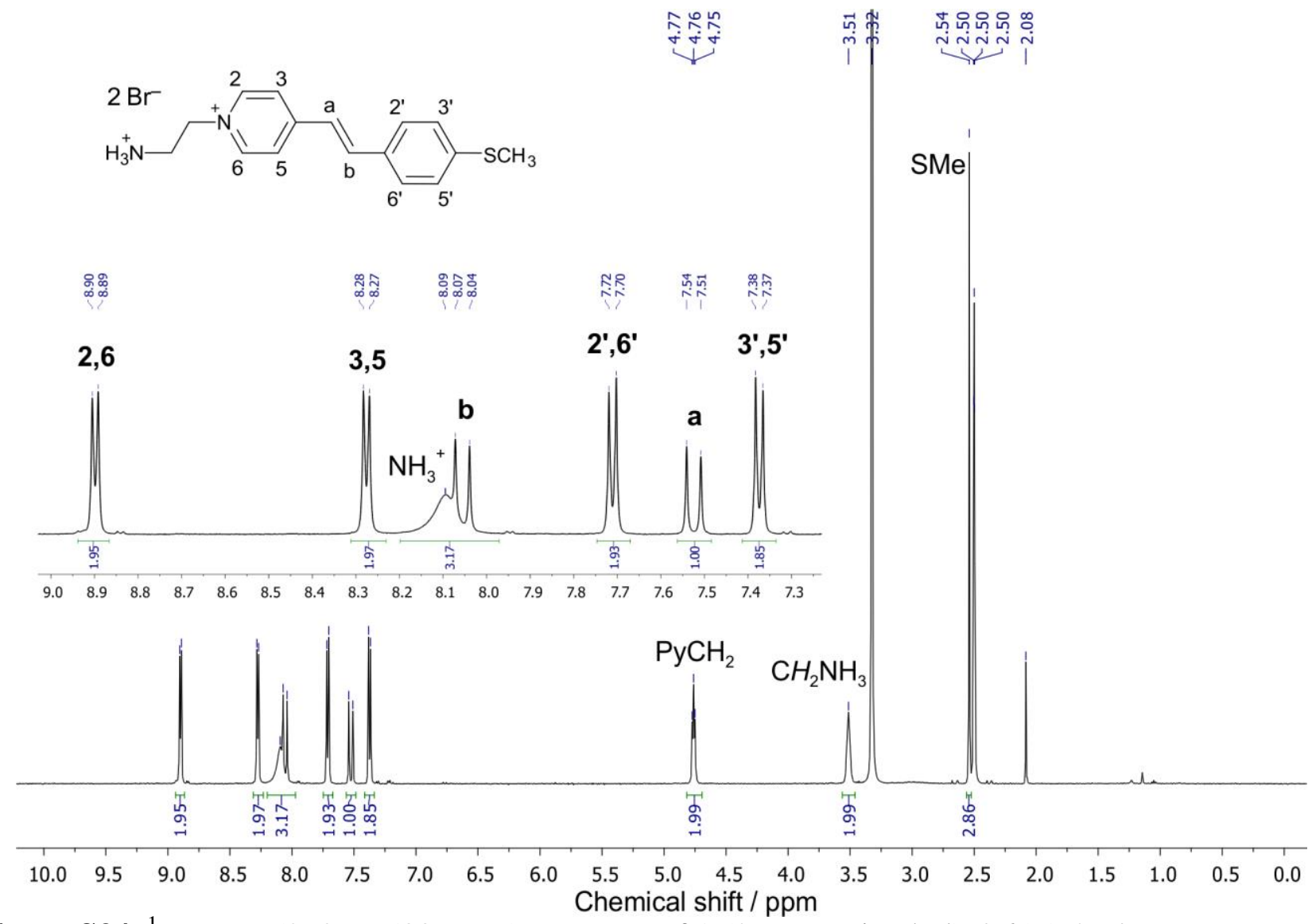

Figure S34. ${ }^{1} \mathrm{H}$ NMR $(298 \mathrm{~K}, 500 \mathrm{MHz})$ spectrum of 1-(2-ammonioethyl)-4- $\{(E)-2-[4-$ (methylthio)phenyl]vinyl $\}$ pyridinium dibromide in DMSO- $d_{6}$. 

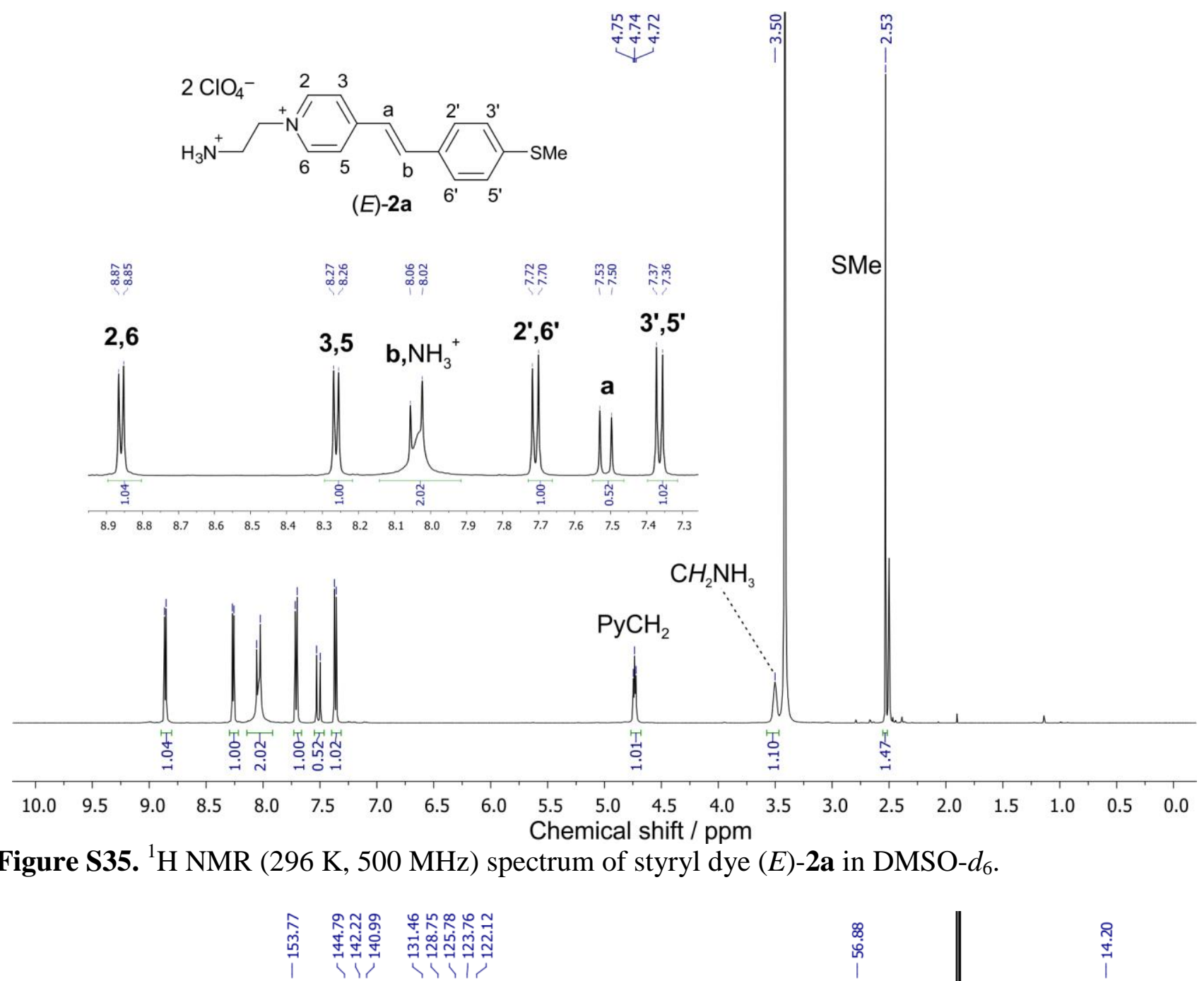

Figure S35. ${ }^{1} \mathrm{H}$ NMR $(296 \mathrm{~K}, 500 \mathrm{MHz})$ spectrum of styryl dye (E)-2a in DMSO- $d_{6}$.

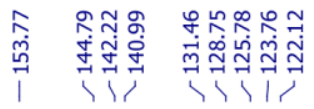

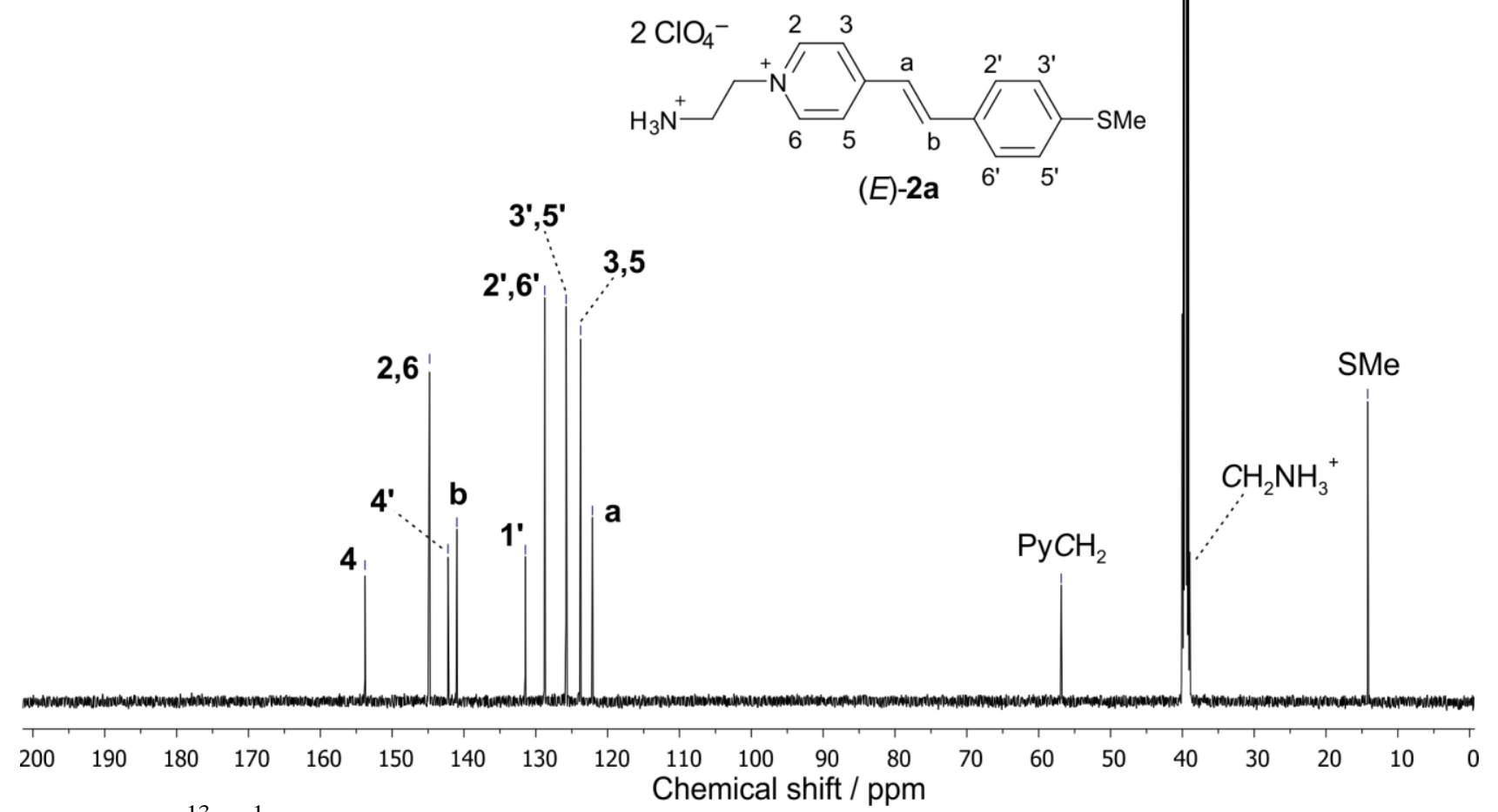

Figure S36. ${ }^{13} \mathrm{C}\left\{{ }^{1} \mathrm{H}\right\}$ NMR $(296 \mathrm{~K}, 125 \mathrm{MHz})$ spectrum of styryl dye $(E)-2 \mathbf{a}$ in DMSO- $d_{6}$. 


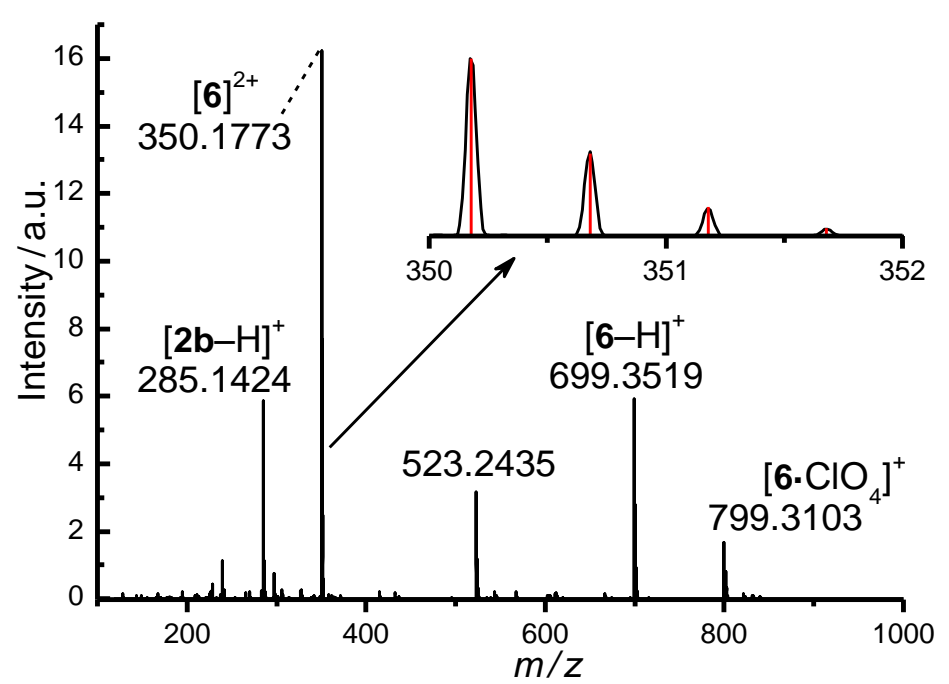

Figure S37. The average ESI mass spectrum of cyclobutane derivative $r c t t-6$ in $\mathrm{MeCN}$ in the positiveion mode; red lines are the calculated isotopic distribution. 
XI Table S9: Cartesian coordinates from DFT calculations in solution

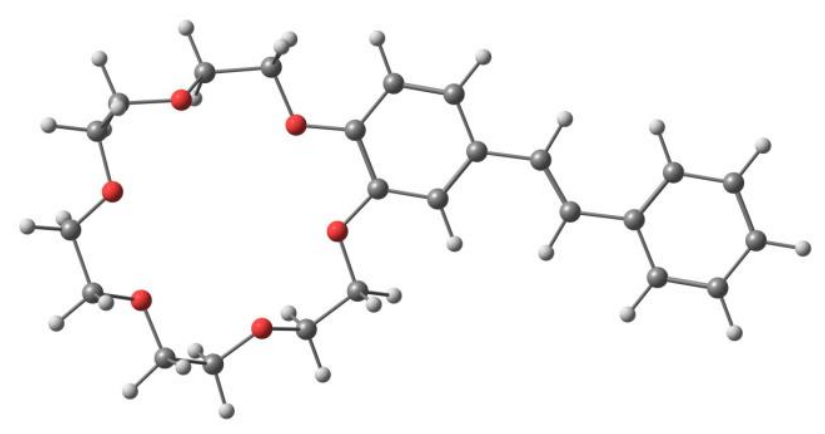

$(u p-s c)-(E)-\mathbf{1}$

Cartesian coordinates $(\AA)$ :

\begin{tabular}{|c|c|c|c|}
\hline J & -0.099055000 & 1.929308000 & -0.987176000 \\
\hline & 2.054080000 & 2.772929000 & -0.418002000 \\
\hline & 1.057253000 & 2.799406000 & -1.410679000 \\
\hline & 4.766814000 & 2.135623000 & 0.212734000 \\
\hline & 4.233248000 & 3.437622000 & 0.278930000 \\
\hline & 3.154684000 & 3.582616000 & -0.766074000 \\
\hline & 5.405448000 & -0.317285000 & 1.599615000 \\
\hline 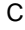 & 6.350225000 & 0.544360000 & 1.001961000 \\
\hline 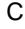 & 5.850778000 & 1.965254000 & 1.096426000 \\
\hline U & -0.558347000 & -0.377190000 & -0.671006000 \\
\hline & -0.044812000 & -1.700670000 & -0.630732000 \\
\hline & 1.285041000 & -1.817568000 & -0.850434000 \\
\hline C & 1.844108000 & -3.121969000 & -0.780095000 \\
\hline C & 3.339398000 & -2.999741000 & -0.933371000 \\
\hline U & 3.863925000 & -2.382812000 & 0.217776000 \\
\hline C & 5.272251000 & -2.307377000 & 0.186763000 \\
\hline C & 5.751340000 & -1.681911000 & 1.481624000 \\
\hline $\mathrm{H}$ & -0.465188000 & 2.231877000 & 0.002480000 \\
\hline $\mathrm{H}$ & -0.914349000 & 2.040106000 & -1.713486000 \\
\hline $\mathrm{H}$ & 0.684913000 & 3.824430000 & -1.564830000 \\
\hline ᄀ & 1.457139000 & 2.438604000 & -2.370761000 \\
\hline H & 3.815432000 & 3.635595000 & 1.278365000 \\
\hline $\mathrm{H}$ & 5.017480000 & 4.187455000 & 0.088206000 \\
\hline-1 & 2.851047000 & 4.639277000 & -0.831806000 \\
\hline-1 & 3.555140000 & 3.285614000 & -1.747633000 \\
\hline $\mathrm{H}$ & 6.514477000 & 0.289729000 & -0.055159000 \\
\hline H & 7.317745000 & 0.465848000 & 1.521678000 \\
\hline $\mathrm{H}$ & 5.546041000 & 2.180450000 & 2.132512000 \\
\hline $\mathrm{H}$ & 6.670420000 & 2.653640000 & 0.836223000 \\
\hline $\mathrm{H}$ & 1.434688000 & -3.752658000 & -1.579486000 \\
\hline $\mathrm{H}$ & 1.611210000 & -3.582852000 & 0.187860000 \\
\hline $\mathrm{H}$ & 3.761002000 & -4.009214000 & -1.059917000 \\
\hline $\mathrm{H}$ & 3.580130000 & -2.418158000 & 2000 \\
\hline-1 & 5.707830000 & -3.315209000 & 0.091979000 \\
\hline-1 & 5.607239000 & -1.718254000 & -0.681340000 \\
\hline $\mathrm{H}$ & 5.274874000 & -2.204190000 & 2.317957000 \\
\hline $\mathrm{H}$ & 6.839371000 & -1.815788000 & 1.570982000 \\
\hline C & -0.910412000 & -2.756074000 & -0.377212000 \\
\hline $\mathrm{H}$ & -0.540312000 & -3.774291000 & -0.352210000 \\
\hline C & -1.903593000 & -0.150050000 & -0.435430000 \\
\hline $\mathrm{H}$ & -2.282673000 & 0.864903000 & -0.455724000 \\
\hline C & -2.785019000 & -1.220732000 & -0.170372000 \\
\hline C & -2.268695000 & -2.513678000 & -0.150062000 \\
\hline $\mathrm{O}$ & 0.354477000 & 0.584611000 & -0.951258000 \\
\hline $\mathrm{H}$ & -2.930774000 & -3.352042000 & 0.048056000 \\
\hline C & -4.218452000 & -1.028881000 & 0.080131000 \\
\hline $\mathrm{H}$ & -4.761947000 & -1.953533000 & 0.270212000 \\
\hline C & -4.881947000 & 0.138896000 & 0.091854000 \\
\hline $\mathrm{H}$ & -4.341859000 & 1.065790000 & -0.096525000 \\
\hline C & -6.319890000 & 0.317574000 & 870000 \\
\hline C & -7.201541000 & -0.748315000 & 0.590147000 \\
\hline$\Omega$ & -6.843749000 & 1.619212000 & 0.334815000 \\
\hline C & -8.552065000 & -0.514539000 & 0.820456000 \\
\hline C & -8.196527000 & 1.853748000 & 0.565569000 \\
\hline C & -9.058076000 & 0.786743000 & 0.809768000 \\
\hline & -6.833043000 & -1.769763000 & 0.602960000 \\
\hline & -6.174698000 & 2.454880000 & 0.144252000 \\
\hline & -9.215711000 & -1.353365000 & 1.009495000 \\
\hline & -8.576559000 & 2.871115000 & 0.554022000 \\
\hline & 971000 & 0.964347000 & 0.989963000 \\
\hline
\end{tabular}

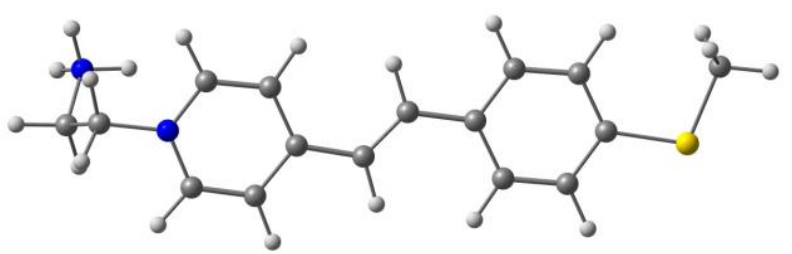

(gauche-st)-(E)-2a

Cartesian coordinates $(\AA)$ :

$\begin{array}{rrrr}\mathrm{C} & 6.075245000 & 0.035929000 & -0.685646000 \\ \mathrm{C} & 6.788039000 & -0.012554000 & 0.653269000 \\ \mathrm{~N} & 6.454932000 & 1.171387000 & 1.500741000 \\ \mathrm{H} & 6.261931000 & 0.981316000 & -1.198083000 \\ \mathrm{H} & 6.445998000 & -0.781022000 & -1.306797000 \\ \mathrm{H} & 7.864192000 & 0.007795000 & 0.482954000 \\ \mathrm{H} & 6.527065000 & -0.903443000 & 1.225978000 \\ \mathrm{H} & 5.462436000 & 1.194515000 & 1.768558000 \\ \mathrm{H} & 6.673265000 & 2.054988000 & 1.022187000 \\ \mathrm{H} & 6.998166000 & 1.153761000 & 2.373257000 \\ \mathrm{~N} & 4.612406000 & -0.125726000 & -0.551244000 \\ \mathrm{C} & 1.861653000 & -0.453669000 & -0.287428000 \\ \mathrm{C} & 3.786927000 & 0.931406000 & -0.727388000 \\ \mathrm{C} & 4.108479000 & -1.344997000 & -0.255000000 \\ \mathrm{C} & 2.754461000 & -1.530142000 & -0.119652000 \\ \mathrm{C} & 2.425716000 & 0.797832000 & -0.600712000 \\ \mathrm{H} & 4.260339000 & 1.874747000 & -0.972779000 \\ \mathrm{H} & 4.828194000 & -2.148242000 & -0.141062000 \\ \mathrm{H} & 2.383142000 & -2.521613000 & 0.113957000 \\ \mathrm{H} & 1.816057000 & 1.679609000 & -0.753937000 \\ \mathrm{C} & 0.434765000 & -0.692053000 & -0.137986000 \\ \mathrm{H} & 0.161663000 & -1.719755000 & 0.086741000 \\ \mathrm{C} & -0.513429000 & 0.258861000 & -0.257595000 \\ \mathrm{H} & -0.217047000 & 1.282611000 & -0.479892000 \\ \mathrm{C} & -1.953609000 & 0.067570000 & -0.116917000 \\ \mathrm{C} & -2.794494000 & 1.176769000 & -0.274898000 \\ \mathrm{C} & -2.546868000 & -1.176029000 & 0.170629000 \\ \mathrm{C} & -4.175820000 & 1.068186000 & -0.154392000 \\ \mathrm{C} & -3.918537000 & -1.294200000 & 0.293332000 \\ \mathrm{C} & -4.751482000 & -0.172427000 & 0.132330000 \\ \mathrm{H} & -2.357197000 & 2.146476000 & -0.498810000 \\ \mathrm{H} & -1.933150000 & -2.061970000 & 0.301030000 \\ \mathrm{H} & -4.788903000 & 1.952671000 & -0.285956000 \\ \mathrm{H} & -4.355515000 & -2.263872000 & 0.517305000 \\ \mathrm{~S} & -6.488390000 & -0.440830000 & 0.311144000 \\ \mathrm{C} & -7.185705000 & 1.208954000 & 0.052280000 \\ \mathrm{H} & -8.265492000 & 1.096951000 & 0.171282000 \\ \mathrm{H} & -6.818501000 & 1.916382000 & 0.798662000 \\ \mathrm{H} & -6.975834000 & 1.575132000 & -0.954739000\end{array}$




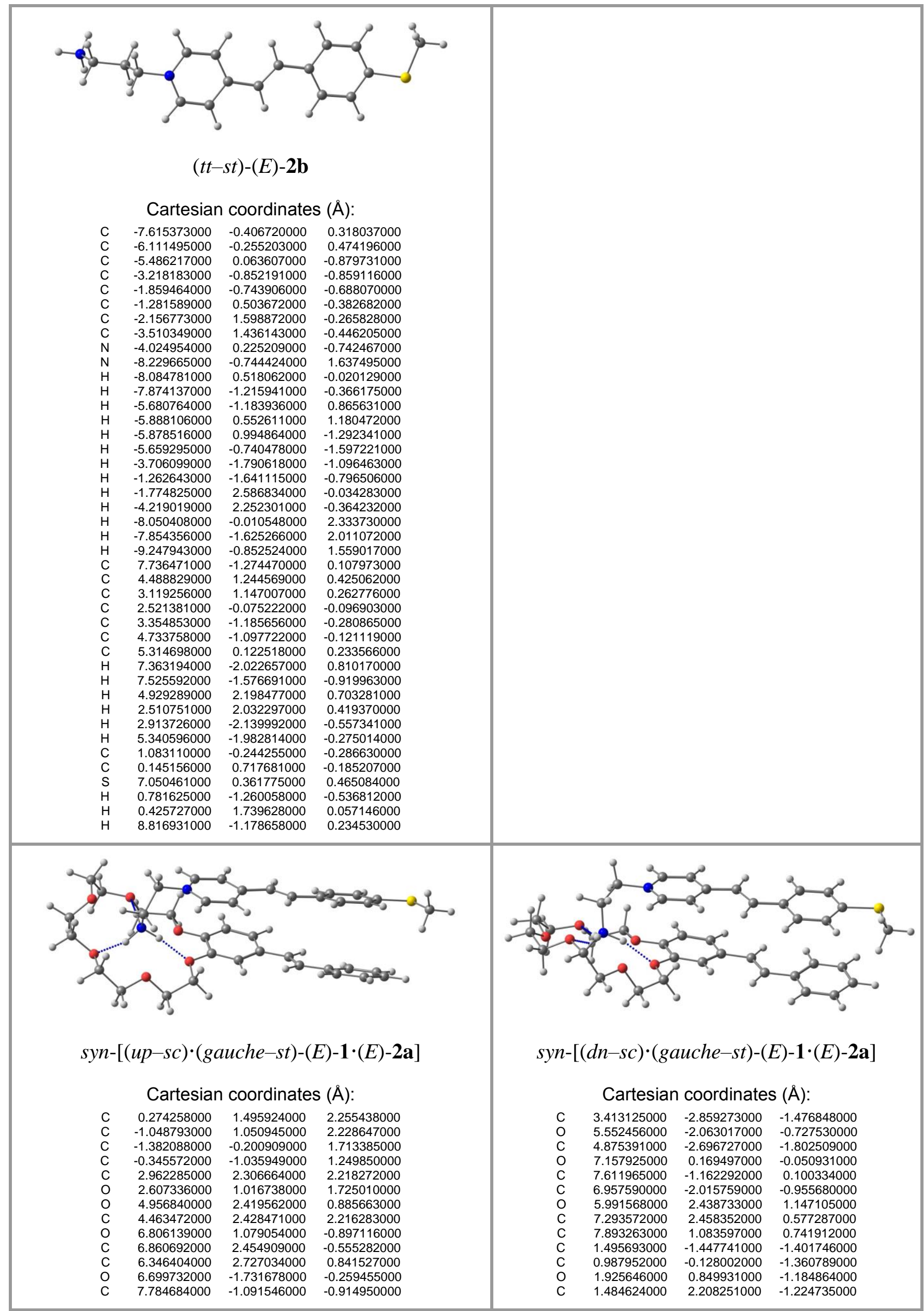




\begin{tabular}{|c|c|c|c|c|c|c|c|}
\hline C & 7.905598000 & 0.325587000 & -0.410216000 & C & 2.696835000 & 3.099556000 & -1.309919000 \\
\hline C & 1.290079000 & 0.677473000 & 1.780555000 & $\mathrm{O}$ & 3.460483000 & 2.988802000 & -0.125215000 \\
\hline C & 0.970947000 & -0.612955000 & 1.297723000 & C & 4.527757000 & 3.924239000 & -0.106046000 \\
\hline $\mathrm{O}$ & 2.046385000 & -1.360709000 & 0.907678000 & C & 5.333666000 & 3.699674000 & 1.153240000 \\
\hline C & 1.848253000 & -2.733886000 & 0.567603000 & $\mathrm{H}$ & 3.265456000 & -3.474027000 & -0.579155000 \\
\hline C & 3.149172000 & -3.468533000 & 0.777724000 & $\mathrm{H}$ & 2.936212000 & -3.354677000 & -2.329199000 \\
\hline $\mathrm{O}$ & 4.152554000 & -2.951683000 & -0.072808000 & $\mathrm{H}$ & 5.302450000 & -3.692592000 & -1.980289000 \\
\hline C & 5.364603000 & -3.675156000 & 0.061087000 & $\mathrm{H}$ & 4.991471000 & -2.100124000 & -2.717157000 \\
\hline C & 6.430830000 & -3.023263000 & -0.782489000 & $\mathrm{H}$ & 7.373133000 & -1.539482000 & 1.106650000 \\
\hline C & -2.797243000 & -0.575445000 & 1.633484000 & $\mathrm{H}$ & 8.701409000 & -1.215543000 & -0.034726000 \\
\hline C & -3.310328000 & -1.578890000 & 0.902917000 & $\mathrm{H}$ & 7.370049000 & -3.032236000 & -0.920051000 \\
\hline C & -4.737884000 & -1.904285000 & 0.776827000 & $\mathrm{H}$ & 7.156520000 & -1.590851000 & -1.948546000 \\
\hline C & -5.131199000 & -2.832514000 & -0.199872000 & $\mathrm{H}$ & 7.251778000 & 2.729348000 & -0.486333000 \\
\hline C & -6.471879000 & -3.156714000 & -0.385796000 & $\mathrm{H}$ & 7.918842000 & 3.192822000 & 1.101328000 \\
\hline C & -7.449991000 & -2.563548000 & 0.410859000 & $\mathrm{H}$ & 7.857262000 & 0.788803000 & 1.801314000 \\
\hline C & -7.073618000 & -1.642878000 & 1.391605000 & $\mathrm{H}$ & 8.945476000 & 1.102473000 & 0.424897000 \\
\hline C & -5.734368000 & -1.313696000 & 1.571898000 & $\mathrm{H}$ & 0.868358000 & 2.370973000 & -2.115240000 \\
\hline $\mathrm{H}$ & 0.496996000 & 2.485357000 & 2.636997000 & $\mathrm{H}$ & 0.891407000 & 2.432119000 & -0.329543000 \\
\hline $\mathrm{H}$ & -1.834825000 & 1.704194000 & 2.597634000 & $\mathrm{H}$ & 2.349915000 & 4.133644000 & -1.446729000 \\
\hline $\mathrm{H}$ & -0.582920000 & -2.017385000 & 0.855174000 & $\mathrm{H}$ & 3.303548000 & 2.823704000 & -2.184734000 \\
\hline $\mathrm{H}$ & 2.607459000 & 2.424569000 & 3.248658000 & $\mathrm{H}$ & 4.131332000 & 4.949789000 & -0.112130000 \\
\hline $\mathrm{H}$ & 2.505757000 & 3.084234000 & 1.592516000 & $\mathrm{H}$ & 5.159411000 & 3.798611000 & -0.998649000 \\
\hline $\mathrm{H}$ & 4.912012000 & 1.605633000 & 2.790402000 & $\mathrm{H}$ & 4.662091000 & 3.698142000 & 2.017403000 \\
\hline $\mathrm{H}$ & 4.729788000 & 3.374934000 & 2.705085000 & $\mathrm{H}$ & 6.063514000 & 4.508410000 & 1.279342000 \\
\hline $\mathrm{H}$ & 7.887609000 & 2.833883000 & -0.644495000 & C & -0.371789000 & 0.102340000 & -1.476324000 \\
\hline $\mathrm{H}$ & 6.234308000 & 2.981703000 & -1.282241000 & $\mathrm{H}$ & -0.751633000 & 1.115359000 & -1.412036000 \\
\hline $\mathrm{H}$ & 6.884444000 & 2.120519000 & 1.582920000 & C & 0.613076000 & -2.508565000 & -1.550295000 \\
\hline $\mathrm{H}$ & 6.498029000 & 3.785429000 & 1.092714000 & $\mathrm{H}$ & 0.976532000 & -3.529696000 & -1.552954000 \\
\hline $\mathrm{H}$ & 8.715257000 & -1.635700000 & -0.704863000 & C & -0.756923000 & -2.267376000 & -1.670408000 \\
\hline $\mathrm{H}$ & 7.623208000 & -1.088773000 & -2.002710000 & C & -1.268312000 & -0.970736000 & -1.648856000 \\
\hline $\mathrm{H}$ & 7.933746000 & 0.323521000 & 0.688073000 & 0 & 2.848118000 & -1.564551000 & -1.274803000 \\
\hline $\mathrm{H}$ & 8.846903000 & 0.756573000 & -0.777912000 & $\mathrm{H}$ & -1.435280000 & -3.108476000 & -1.782385000 \\
\hline $\mathrm{H}$ & 1.103432000 & -3.183566000 & 1.231055000 & C & -2.717956000 & -0.778696000 & -1.768424000 \\
\hline $\mathrm{H}$ & 1.499451000 & -2.809014000 & -0.469261000 & $\mathrm{H}$ & -3.291002000 & -1.704699000 & -1.746108000 \\
\hline $\mathrm{H}$ & 3.458290000 & -3.373472000 & 1.828584000 & C & -3.362096000 & 0.393610000 & -1.891269000 \\
\hline $\mathrm{H}$ & 2.978440000 & -4.534161000 & 0.567202000 & $\mathrm{H}$ & -2.790785000 & 1.320583000 & -1.930908000 \\
\hline $\mathrm{H}$ & 5.681274000 & -3.687783000 & 1.113801000 & C & -4.819566000 & 0.569435000 & -1.970801000 \\
\hline $\mathrm{H}$ & 5.226014000 & -4.714139000 & -0.269446000 & C & -5.725670000 & -0.503394000 & -1.915251000 \\
\hline $\mathrm{H}$ & 7.340229000 & -3.638234000 & -0.749050000 & C & -5.336398000 & 1.869131000 & -2.080069000 \\
\hline $\mathrm{H}$ & 6.102419000 & -2.946226000 & -1.829251000 & C & -7.095667000 & -0.278189000 & -1.968486000 \\
\hline $\mathrm{H}$ & -3.467994000 & 0.082702000 & 2.184832000 & C & -6.709820000 & 2.095435000 & -2.129673000 \\
\hline $\mathrm{H}$ & -2.646091000 & -2.200817000 & 0.302971000 & C & -7.596396000 & 1.021344000 & -2.073961000 \\
\hline $\mathrm{H}$ & -4.369632000 & -3.297763000 & -0.821525000 & $\mathrm{H}$ & -5.362358000 & -1.522595000 & -1.822586000 \\
\hline $\mathrm{H}$ & -6.752391000 & -3.876544000 & -1.149181000 & $\mathrm{H}$ & -4.647294000 & 2.709300000 & -2.120231000 \\
\hline $\mathrm{H}$ & -7.828999000 & -1.180776000 & 2.020760000 & $\mathrm{H}$ & -7.779996000 & -1.120262000 & -1.918552000 \\
\hline $\mathrm{H}$ & -5.463541000 & -0.603272000 & 2.347553000 & $\mathrm{H}$ & -7.086637000 & 3.110898000 & -2.212105000 \\
\hline C & 3.563038000 & 0.773587000 & -2.394209000 & $\mathrm{H}$ & -8.668631000 & 1.191346000 & -2.112196000 \\
\hline C & 4.275166000 & -0.372473000 & -1.707665000 & C & 3.721575000 & -1.370347000 & 1.971520000 \\
\hline $\mathrm{N}$ & 4.391252000 & -0.161539000 & -0.243445000 & C & 4.476052000 & -0.093284000 & 1.679494000 \\
\hline $\mathrm{H}$ & 4.079105000 & 1.719439000 & -2.219609000 & $\mathrm{~N}$ & 4.394545000 & 0.314802000 & 0.256769000 \\
\hline $\mathrm{H}$ & 3.532909000 & 0.587533000 & -3.469329000 & $\mathrm{H}$ & 4.055067000 & -2.165907000 & 1.300706000 \\
\hline $\mathrm{H}$ & 5.285221000 & -0.434865000 & -2.114598000 & $\mathrm{H}$ & 3.931648000 & -1.668604000 & 3.001282000 \\
\hline $\mathrm{H}$ & 3.769006000 & -1.327335000 & -1.860376000 & $\mathrm{H}$ & 5.530044000 & -0.283487000 & 1.891581000 \\
\hline $\mathrm{H}$ & 3.531745000 & -0.433301000 & 0.256825000 & $\mathrm{H}$ & 4.165393000 & 0.747501000 & 2.300825000 \\
\hline $\mathrm{H}$ & 4.637497000 & 0.812539000 & 0.012320000 & $\mathrm{H}$ & 3.438535000 & 0.503896000 & -0.079787000 \\
\hline $\mathrm{H}$ & 5.158708000 & -0.771713000 & 0.087366000 & $\mathrm{H}$ & 4.817006000 & -0.421466000 & -0.335923000 \\
\hline $\mathrm{N}$ & 2.167464000 & 0.938627000 & -1.931047000 & $\mathrm{H}$ & 4.942999000 & 1.183987000 & 0.165805000 \\
\hline C & -0.514622000 & 1.328623000 & -1.300051000 & $\mathrm{~N}$ & 2.244395000 & -1.283401000 & 1.834996000 \\
\hline C & 1.796744000 & 2.040609000 & -1.244783000 & C & -0.545972000 & -1.271038000 & 1.765628000 \\
\hline C & 1.250836000 & 0.000740000 & -2.269898000 & C & 1.564823000 & -2.448668000 & 1.750344000 \\
\hline C & -0.075368000 & 0.160651000 & -1.957608000 & C & 1.570937000 & -0.113257000 & 1.894450000 \\
\hline C & 0.475000000 & 2.253550000 & -0.921542000 & C & 0.196908000 & -0.078600000 & 1.853955000 \\
\hline $\mathrm{H}$ & 2.587292000 & 2.737129000 & -0.986553000 & C & 0.191112000 & -2.468785000 & 1.721736000 \\
\hline $\mathrm{H}$ & 1.624347000 & -0.861575000 & -2.811042000 & $\mathrm{H}$ & 2.167600000 & -3.349186000 & 1.714603000 \\
\hline $\mathrm{H}$ & -0.762401000 & -0.627545000 & -2.241478000 & $\mathrm{H}$ & 2.152402000 & 0.795290000 & 1.982385000 \\
\hline $\mathrm{H}$ & 0.207562000 & 3.158201000 & -0.386565000 & $\mathrm{H}$ & -0.282375000 & 0.891639000 & 1.908172000 \\
\hline $\mathrm{H}$ & -8.496221000 & -2.823178000 & 0.278405000 & $\mathrm{H}$ & -0.315340000 & -3.425113000 & 1.650912000 \\
\hline C & -1.908797000 & 1.604130000 & -0.992314000 & C & -1.999256000 & -1.329911000 & 1.723720000 \\
\hline $\mathrm{H}$ & -2.079453000 & 2.471492000 & -0.359020000 & $\mathrm{H}$ & -2.419006000 & -2.331589000 & 1.773354000 \\
\hline C & -2.944012000 & 0.868377000 & -1.444904000 & C & -2.802127000 & -0.253544000 & 1.605636000 \\
\hline $\mathrm{H}$ & -2.753571000 & 0.036122000 & -2.121473000 & $\mathrm{H}$ & -2.355184000 & 0.737216000 & 1.529021000 \\
\hline C & -4.353201000 & 1.071231000 & -1.121315000 & C & -4.261949000 & -0.257698000 & 1.553681000 \\
\hline C & -5.317395000 & 0.319670000 & -1.805084000 & C & -4.928870000 & 0.969394000 & 1.445589000 \\
\hline C & -4.792093000 & 1.953234000 & -0.117607000 & C & -5.040444000 & -1.429282000 & 1.599260000 \\
\hline C & -6.674384000 & 0.447291000 & -1.527444000 & C & -6.316943000 & 1.046119000 & 1.395526000 \\
\hline C & -6.138895000 & 2.082347000 & 0.171964000 & C & -6.421511000 & -1.364004000 & 1.549231000 \\
\hline C & -7.097223000 & 1.332032000 & -0.531675000 & C & -7.078237000 & -0.124217000 & 1.448740000 \\
\hline $\mathrm{H}$ & -4.996949000 & -0.380791000 & -2.572723000 & $\mathrm{H}$ & -4.345908000 & 1.886201000 & 1.396697000 \\
\hline $\mathrm{H}$ & -4.073456000 & 2.532411000 & 0.455292000 & $\mathrm{H}$ & -4.565080000 & -2.403111000 & 1.671857000 \\
\hline $\mathrm{H}$ & -7.386492000 & -0.154115000 & -2.081552000 & $\mathrm{H}$ & -6.788764000 & 2.018566000 & 1.310714000 \\
\hline $\mathrm{H}$ & -6.456684000 & 2.762010000 & 0.958406000 & $\mathrm{H}$ & -7.003375000 & -2.281437000 & 1.583216000 \\
\hline S & -8.786357000 & 1.564624000 & -0.068206000 & $\mathrm{~S}$ & -8.842783000 & -0.156651000 & 1.378231000 \\
\hline C & -9.654512000 & 0.352045000 & -1.092619000 & C & -9.269828000 & 1.593964000 & 1.215849000 \\
\hline $\mathrm{H}$ & -10.707132000 & 0.418669000 & -0.809113000 & $\mathrm{H}$ & -10.358082000 & 1.631187000 & 1.132225000 \\
\hline $\mathrm{H}$ & -9.558383000 & 0.583393000 & -2.155399000 & $\mathrm{H}$ & -8.961227000 & 2.161613000 & 2.096294000 \\
\hline $\mathrm{H}$ & -9.294080000 & -0.658927000 & -0.887743000 & $\mathrm{H}$ & -8.827627000 & 2.022863000 & 0.313274000 \\
\hline
\end{tabular}




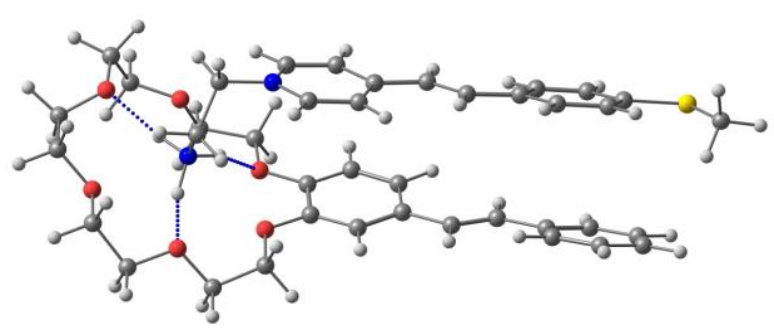

anti-[(up-st) $\cdot($ gauche $-s t)-(E)-\mathbf{1} \cdot(E)-\mathbf{2 a}]$

Cartesian coordinates $(\AA)$ :

$-0.100940000-0.704186000$

2.148968000

$\begin{array}{lll}1.145262000 & -0.144340000 & 1.875798000\end{array}$

$\begin{array}{lll}1.254286000 & 1.066458000 & 1.188833000\end{array}$

$\begin{array}{lll}0.072873000 & 1.730983000 & 0.807375000\end{array}$

$\begin{array}{lll}-2.656181000 & -1.829082000 & 2.523732000\end{array}$

$\begin{array}{lll}-2.523944000 & -0.551060000 & 1.902025000\end{array}$

$\begin{array}{lll}-4.625687000 & -2.335382000 & 1.277345000\end{array}$

$\begin{array}{lll}-4.122259000 & -2.169909000 & 2.589866000\end{array}$

$\begin{array}{lll}-6.569357000 & -1.560174000 & -0.695491000\end{array}$

$\begin{array}{lll}-6.453497000 & -2.868164000 & -0.143830000\end{array}$

$\begin{array}{lll}-5.951471000 & -2.841162000 & 1.282259000\end{array}$

$\begin{array}{lll}-6.927881000 & 1.274199000 & -0.436431000\end{array}$

$\begin{array}{lll}-7.843782000 & 0.418392000 & -1.094526000\end{array}$

$\begin{array}{lll}-7.821386000 & -0.935846000 & -0.429987000\end{array}$

$\begin{array}{lll}-1.261296000 & -0.056872000 & 1.742439000\end{array}$

$\begin{array}{lll}-1.174012000 & 1.196562000 & 1.100328000\end{array}$

$\begin{array}{lll}-2.367775000 & 1.789258000 & 0.816665000\end{array}$

$\begin{array}{lll}-2.357706000 & 3.122843000 & 0.314988000\end{array}$

$\begin{array}{lll}-3.744327000 & 3.692207000 & 0.493257000\end{array}$

$\begin{array}{lll}-4.669982000 & 2.931438000 & -0.263915000\end{array}$

$\begin{array}{lll}-5.991907000 & 3.444605000 & -0.180580000\end{array}$

$\begin{array}{lll}-6.902625000 & 2.570022000 & -1.006276000\end{array}$

$\begin{array}{lll}2.546678000 & 1.652248000 & 0.808169000\end{array}$

$\begin{array}{lll}3.749620000 & 1.219547000 & 1.221293000\end{array}$

$\begin{array}{lll}5.054294000 & 1.745236000 & 0.796404000\end{array}$

$\begin{array}{lll}6.201421000 & 1.352045000 & 1.500805000\end{array}$

$\begin{array}{lll}7.464391000 & 1.804734000 & 1.125833000\end{array}$

$\begin{array}{lll}7.603131000 & 2.654316000 & 0.029738000\end{array}$

$\begin{array}{lll}6.470113000 & 3.045076000 & -0.688146000\end{array}$

$\begin{array}{lll}5.210407000 & 2.594914000 & -0.311928000\end{array}$

$\begin{array}{lll}-0.155648000 & -1.659169000 & 2.658773000\end{array}$

$\begin{array}{lll}2.034380000 & -0.688232000 & 2.178384000\end{array}$

$\begin{array}{lll}0.148152000 & 2.674484000 & 0.276431000\end{array}$

$\begin{array}{lll}-2.244368000 & -1.791520000 & 3.538413000\end{array}$

$\begin{array}{lll}-2.111752000 & -2.585250000 & 1.944254000\end{array}$

$\begin{array}{lll}-4.675018000 & -1.377218000 & 3.113881000\end{array}$

$\begin{array}{lll}-4.232197000 & -3.101449000 & 3.162345000\end{array}$

$\begin{array}{lll}-7.419959000 & -3.384240000 & -0.190385000\end{array}$

$\begin{array}{lll}-5.739937000 & -3.410344000 & -0.772213000\end{array}$

$\begin{array}{lll}-6.594794000 & -2.207692000 & 1.910583000\end{array}$

$\begin{array}{lll}-5.968512000 & -3.859332000 & 1.696497000\end{array}$

$\begin{array}{lll}-8.861301000 & 0.829463000 & -1.030320000\end{array}$

$\begin{array}{lll}-7.580268000 & 0.318285000 & -2.158418000\end{array}$

$\begin{array}{lll}-7.979754000 & -0.824387000 & 0.650575000\end{array}$

$\begin{array}{lll}-8.630890000 & -1.553096000 & -0.840099000\end{array}$

$\begin{array}{lll}-1.653911000 & 3.740543000 & 0.883935000\end{array}$

$\begin{array}{lll}-2.060141000 & 3.123756000 & -0.741041000\end{array}$

$\begin{array}{lll}-4.021940000 & 3.674967000 & 1.556067000\end{array}$

$\begin{array}{lll}-3.743213000 & 4.735796000 & 0.150612000\end{array}$

$\begin{array}{lll}-6.324732000 & 3.456108000 & 0.866460000\end{array}$

$\begin{array}{lll}-6.021094000 & 4.471591000 & -0.568150000\end{array}$

$\begin{array}{lll}-7.911147000 & 3.007095000 & -1.010472000\end{array}$

$\begin{array}{lll}-6.544066000 & 2.523464000 & -2.045731000\end{array}$

$\begin{array}{llll}2.485601000 & 2501436000 & 0.128297000\end{array}$

$\begin{array}{lll}3.806562000 & 0.403242000 & 1.940611000\end{array}$

$\begin{array}{lll}6.096156000 & 0.681350000 & 2.350536000\end{array}$

$8.339739000 \quad 1.488775000 \quad 1.686152000$

$6.572158000 \quad 3.698361000-1.549789000$

$\begin{array}{lll}4.342360000 & 2.889428000 & -0.895226000\end{array}$

$\begin{array}{lll}-3.576211000 & -0.983720000 & -2.264998000\end{array}$

$\begin{array}{lll}-4.166040000 & 0.279493000 & -1.666659000\end{array}$

$\begin{array}{lll}-4.403335000 & 0.148676000 & -0.211246000\end{array}$

$\begin{array}{lll}-4.134120000 & -1.871987000 & -1.963385000\end{array}$

$\begin{array}{lll}-3.595441000 & -0.911188000 & -3.353578000\end{array}$

$\begin{array}{lll}-5.134139000 & 0.477064000 & -2.131360000\end{array}$

$\begin{array}{lll}-3.519358000 & 1.143799000 & -1.830413000\end{array}$

$\begin{array}{llll}-3.581738000 & -0.155095000 & 0.330383000\end{array}$

$\begin{array}{lll}-5.173095000 & -0.522786000 & -0.052889000\end{array}$

$\begin{array}{lll}-4.678293000 & 1.079421000 & 0.140960000\end{array}$

$\begin{array}{lll}-2.166821000 & -1.175729000 & -1.858134000\end{array}$

$\begin{array}{lll}0.510946000 & -1.521804000 & -1.183007000\end{array}$

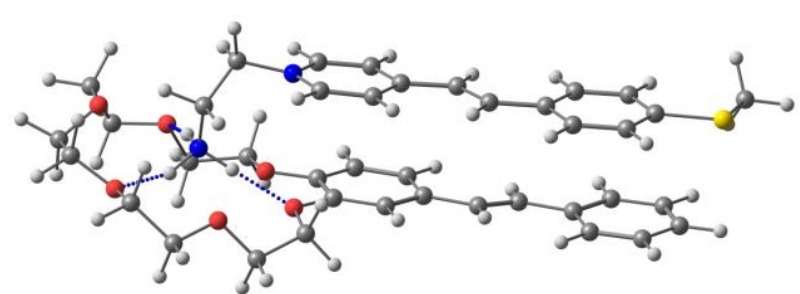

anti-[(up-st $) \cdot($ gauche $-s c)-(E)-\mathbf{1} \cdot(E)-\mathbf{2 a}]$

Cartesian coordinates $(\AA)$ :

\begin{tabular}{|c|c|c|c|}
\hline & 0.516380000 & 2.774328000 & \\
\hline & 574000 & 499051000 & 920 \\
\hline & -1.246105000 & 257103000 & 1.1279030 \\
\hline & -0.265887000 & 277810000 & 1.37942900 \\
\hline & & 5797000 & \\
\hline & 2.820847000 & 971913000 & 0.5146400 \\
\hline & 5.360407000 & 449784000 & -0.6865790 \\
\hline & 4.779056000 & 3.305182000 & 0.284303 \\
\hline & 7.118585000 & 0.198495000 & -1.1943810 \\
\hline & 7.327692000 & & \\
\hline & 6.779037000 & 2.560249000 & -0.7064980 \\
\hline & 6.696105000 & 1707276000 & \\
\hline & & & \\
\hline & 8.138684000 & -0.264429000 & 249710 \\
\hline & 1.481436000 & 1.809185000 & 0.6945150 \\
\hline & & & \\
\hline & 2.0 & 14000 & \\
\hline & 1.79 & -1.57 & \\
\hline & 3.0 & -2.0 & \\
\hline & & & \\
\hline & & & \\
\hline & $6.2 \varepsilon$ & -3.0 & \\
\hline & & & \\
\hline & & & \\
\hline & -5.1 & & \\
\hline & & & \\
\hline & -7.4 & & \\
\hline & -7.8 & & \\
\hline & & & \\
\hline & -5.5 & & \\
\hline & & & \\
\hline & -1.5 & & \\
\hline & -0.5 & & \\
\hline & & & \\
\hline & & & \\
\hline & & & \\
\hline & & & \\
\hline & & & \\
\hline & & & \\
\hline & & & \\
\hline & & & \\
\hline & & & \\
\hline & & & \\
\hline & & & \\
\hline & & & \\
\hline & & & \\
\hline & & & \\
\hline & & & \\
\hline & & & \\
\hline & & & \\
\hline & & & \\
\hline & & & \\
\hline & & & \\
\hline & & & \\
\hline & & & \\
\hline & & & \\
\hline & & & \\
\hline & & & \\
\hline & & & \\
\hline & & & \\
\hline & & & \\
\hline & & & \\
\hline & & & \\
\hline & & & \\
\hline & & & \\
\hline & & & \\
\hline & & & \\
\hline & & & \\
\hline & & & \\
\hline & & & \\
\hline
\end{tabular}




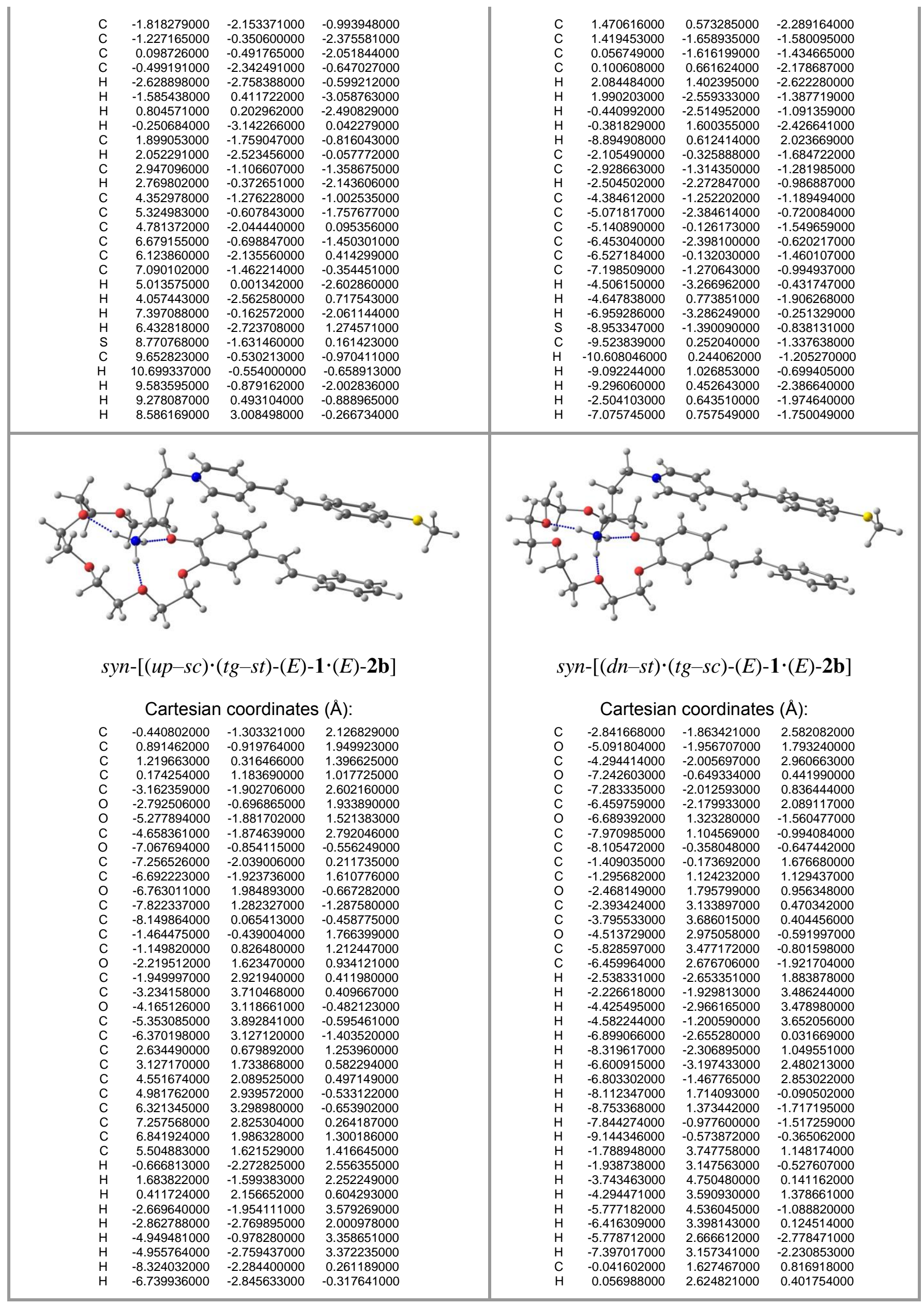




\begin{tabular}{|c|c|c|c|c|c|c|c|}
\hline $\begin{array}{l}\mathrm{H} \\
\mathrm{H} \\
\mathrm{H} \\
\mathrm{H} \\
\mathrm{H} \\
\mathrm{H} \\
\mathrm{H} \\
\mathrm{H} \\
\mathrm{H} \\
\mathrm{H} \\
\mathrm{H} \\
\mathrm{H} \\
\mathrm{H} \\
\mathrm{H} \\
\mathrm{H} \\
\mathrm{H} \\
\mathrm{H} \\
\mathrm{H} \\
\mathrm{H} \\
\mathrm{H} \\
\mathrm{C} \\
\mathrm{C} \\
\mathrm{C} \\
\mathrm{N} \\
\mathrm{H} \\
\mathrm{H} \\
\mathrm{H} \\
\mathrm{H} \\
\mathrm{H} \\
\mathrm{H} \\
\mathrm{H} \\
\mathrm{H} \\
\mathrm{H} \\
\mathrm{H} \\
\mathrm{H} \\
\mathrm{H} \\
\mathrm{C} \\
\mathrm{C} \\
\mathrm{C} \\
\mathrm{H} \\
\mathrm{H} \\
\mathrm{C} \\
\mathrm{C} \\
\mathrm{C} \\
\mathrm{H} \\
\mathrm{H} \\
\mathrm{C} \\
\mathrm{H} \\
\mathrm{H} \\
\mathrm{C} \\
\mathrm{H} \\
\mathrm{C} \\
\mathrm{H} \\
\mathrm{C} \\
\mathrm{C} \\
\mathrm{C} \\
\mathrm{C} \\
\\
\mathrm{H}\end{array}$ & 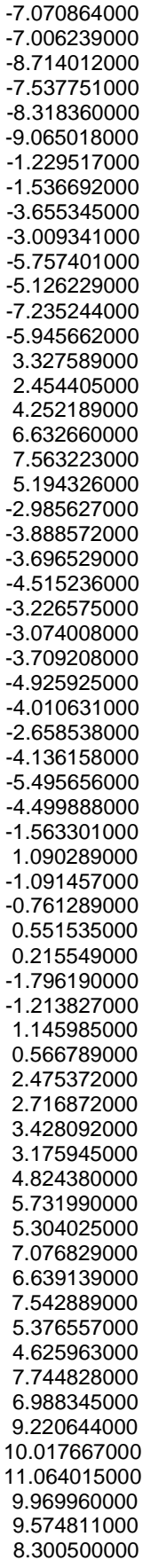 & 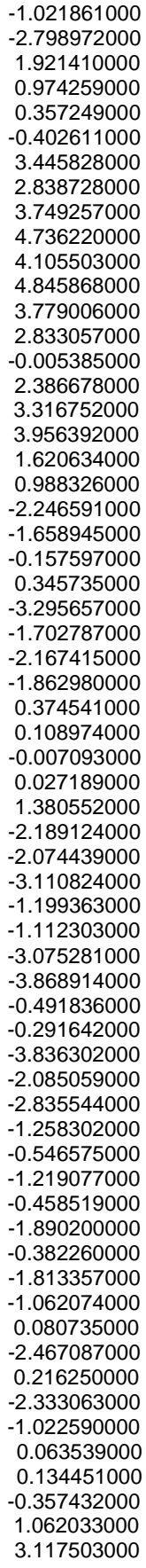 & 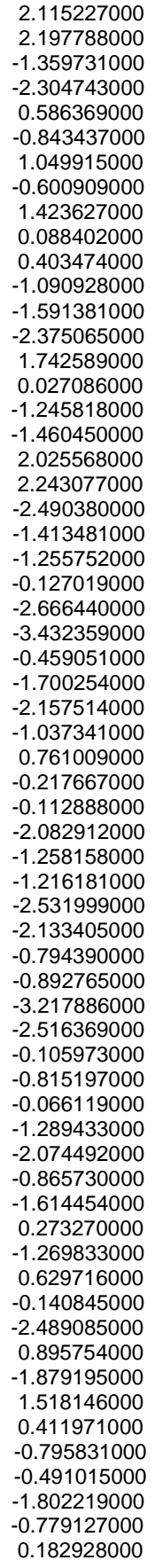 & 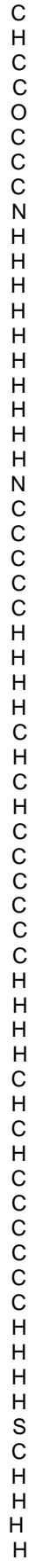 & $\begin{array}{r}-0.263713000 \\
-0.340813000 \\
0.993985000 \\
1.125196000 \\
-2.676468000 \\
-3.016016000 \\
-3.852315000 \\
-3.852543000 \\
-4.608929000 \\
-3.114380000 \\
-3.308092000 \\
-3.490377000 \\
-4.877451000 \\
-4.327279000 \\
-2.841059000 \\
-4.124435000 \\
-5.572646000 \\
-4.666281000 \\
1.871933000 \\
-1.576287000 \\
1.126097000 \\
-0.868462000 \\
-0.982850000 \\
0.351455000 \\
0.466099000 \\
-1.414365000 \\
-1.609216000 \\
0.798435000 \\
0.983912000 \\
2.424704000 \\
2.380142000 \\
3.612261000 \\
3.649939000 \\
4.914831000 \\
5.112398000 \\
6.012283000 \\
6.362967000 \\
7.264682000 \\
7.444266000 \\
4.286304000 \\
5.874605000 \\
6.498918000 \\
8.099669000 \\
2.541443000 \\
2.906719000 \\
3.374705000 \\
2.983297000 \\
4.801408000 \\
5.474435000 \\
5.540389000 \\
6.825143000 \\
6.896826000 \\
7.553635000 \\
4.922228000 \\
5.059794000 \\
7.320240000 \\
9.2709040000 \\
\end{array}$ & 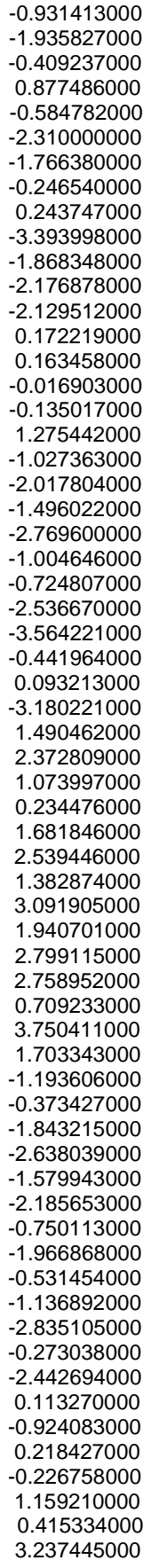 & 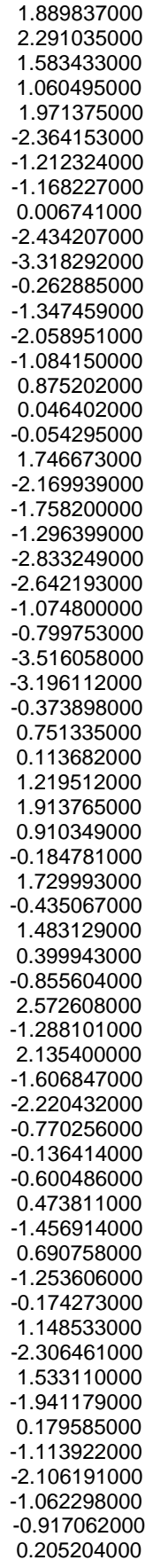 \\
\hline $\begin{array}{l}\text { C } \\
\text { O } \\
\text { C } \\
\text { O } \\
\text { C }\end{array}$ & $\begin{array}{c}i-[(u p-s t) \cdot \\
\text { Cartesiar } \\
-2.361764000 \\
-4.632142000 \\
-3.721899000 \\
-7.100306000 \\
-6.875616000\end{array}$ & $\begin{array}{c}\text { coordinate } \\
3.161314000 \\
3.045509000 \\
3.808572000 \\
1.639261000 \\
2.736340000\end{array}$ & $\begin{array}{l}\mathbf{1} \cdot(E)-\mathbf{2 b}] \\
(\AA): \\
0.009208000 \\
-0.738867000 \\
0.036243000 \\
-0.636458000 \\
-1.500828000\end{array}$ & $\begin{array}{l}\text { C } \\
\text { O } \\
\text { C } \\
\text { O } \\
\text { C }\end{array}$ & $\begin{array}{r}\text { Cartesiar } \\
-2.881951000 \\
-5.143567000 \\
-4.333775000 \\
-7.271413000 \\
-7.344718000\end{array}$ & $\begin{array}{l}\text { coordinate } \\
-1.815925000 \\
-1.873959000 \\
-1.932180000 \\
-0.551466000 \\
-1.899031000\end{array}$ & $\begin{array}{l}\mathbf{1}(E)-\mathbf{2 b}] \\
(\AA): \\
2.539965000 \\
1.774240000 \\
2.932757000 \\
0.394808000 \\
0.835270000\end{array}$ \\
\hline
\end{tabular}




\begin{tabular}{|c|c|c|c|}
\hline & -5.901912000 & 3.678083000 & -0.838600000 \\
\hline & -7.086419000 & -1.192363000 & 30000 \\
\hline & -8.251472000 & -0.384078000 & -0.161891000 \\
\hline & 124608000 & 34598000 & -1 \\
\hline & -1.294952000 & 1.231786000 & 0.907403000 \\
\hline & -1.428656000 & -0.003033000 & 1.581295000 \\
\hline & -2.705385000 & -0.389366000 & 1.86483000 \\
\hline & -2.890921000 & -1.569089000 & 2.645961000 \\
\hline & -4.363772000 & -1.682841000 & 2.949930000 \\
\hline & 36000 & -1.840478000 & 37000 \\
\hline & 673000 & -2.038182000 & 5000 \\
\hline & 3000 & 2000 & 1000 \\
\hline & & & \\
\hline & & & \\
\hline & & & \\
\hline & & 00 & \\
\hline & 000 & & \\
\hline & 000 & 00 & \\
\hline & 000 & 2000 & -1 \\
\hline & -6.2 & 000 & 000 \\
\hline & & 3000 & 5000 \\
\hline & & & \\
\hline & & & \\
\hline $\mathrm{H}$ & & & \\
\hline & & & \\
\hline & & & \\
\hline & -4.5 & & \\
\hline & -4.7 & -0.7 & \\
\hline & -6.6 & $-2 . \varepsilon$ & 2.5 \\
\hline & -6.5 & 000 & \\
\hline & & & \\
\hline & & & \\
\hline & & & \\
\hline & & & \\
\hline 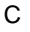 & & & \\
\hline $\mathrm{H}$ & & & \\
\hline C & & & \\
\hline C & 0.9 & -0. & \\
\hline $\mathrm{O}$ & -2.4 & & 00 \\
\hline C & $-2 . \varsigma$ & -2.3 & 1000 \\
\hline C & & -1. & \\
\hline & & & \\
\hline & & & \\
\hline & & & \\
\hline & & & \\
\hline & & 0 & \\
\hline & -3.6 & -2 & \\
\hline-1 & -2.8 & & -1 . \\
\hline $\mathrm{H}$ & $-4.1 \mathrm{c}$ & 0.1 & 98000 \\
\hline $\mathrm{H}$ & -4.2 & & \\
\hline $\mathrm{H}$ & -4.7 & & \\
\hline $\mathrm{H}$ & & & \\
\hline $\mathrm{H}$ & & & \\
\hline & & & \\
\hline 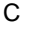 & & & \\
\hline C & & & \\
\hline C & -1.0 & & \\
\hline C & & -3. & \\
\hline C & 0.5 & -1 & -1 \\
\hline $\mathrm{H}$ & -1.2 & -0.5 & -3. \\
\hline $\mathrm{H}$ & -1.7 & & \\
\hline $\mathrm{H}$ & & & \\
\hline $\mathrm{H}$ & & & \\
\hline c & & & \\
\hline $\mathrm{H}$ & & & \\
\hline C & & & \\
\hline $\mathrm{H}$ & & & \\
\hline C & & & \\
\hline $\mathrm{H}$ & & 2.4 & -0. \\
\hline C & & 6000 & \\
\hline $\mathrm{H}$ & & & \\
\hline C & & -1 & \\
\hline C & & & \\
\hline C & & & \\
\hline C & & & \\
\hline C & & & \\
\hline C & & & \\
\hline $\mathrm{H}$ & & & \\
\hline $\mathrm{H}$ & & & \\
\hline $\mathrm{H}$ & & & \\
\hline $\mathrm{H}$ & & & \\
\hline$C$ & & & \\
\hline$C$ & & & \\
\hline C & & & \\
\hline r & & & \\
\hline & & & 1.339682 \\
\hline & 7.454950000 & 2.885527000 & 0.25254400 \\
\hline
\end{tabular}

\begin{tabular}{|c|c|c|c|}
\hline & -6.515099000 & -2.049308000 & $2.0865920 \mathrm{C}$ \\
\hline & -6.655515000 & 1.331287000 & -1.661 \\
\hline & -7.948609000 & 1281000 & 98000 \\
\hline & -8.129040000 & -0.273278000 & -0 . \\
\hline & 1.427334000 & -0.136723000 & 3900 \\
\hline & .299245000 & 1.159318000 & 568900 \\
\hline & 5032000 & 6567000 & 0 \\
\hline & -2 & & \\
\hline & -3.7 & 5000 & 84000 \\
\hline & 32000 & 5000 & 23000 \\
\hline & 5000 & 9000 & 43000 \\
\hline & 7000 & 1000 & 0000 \\
\hline & 4535000 & -2.613488000 & 64000 \\
\hline & 000 & 1000 & 09000 \\
\hline & 000 & 2000 & 8000 \\
\hline & & 000 & \\
\hline & & & \\
\hline & & & \\
\hline & & & \\
\hline & -6.8 & & \\
\hline & $-8 . c$ & 000 & 5000 \\
\hline & -8.7 & 3000 & 5000 \\
\hline & -7.8 & 000 & 5000 \\
\hline & & & 5000 \\
\hline & & & 1 \\
\hline & & & \\
\hline & & & \\
\hline & & & \\
\hline & -5 & & \\
\hline & -6.3 & & -0 \\
\hline & -5.6 & 000 & -2 \\
\hline & -7 & & 5000 \\
\hline & -0. & & 0. \\
\hline $\mathrm{r}$ & & & \\
\hline$c$ & & & \\
\hline & & & \\
\hline$c$ & & & \\
\hline C & & & \\
\hline & $-2.7 c$ & -0 . & 000 \\
\hline $\mathrm{r}$ & 1.8 & -0. & 3000 \\
\hline C & 2.4 & & 000 \\
\hline $\mathrm{H}$ & & & 000 \\
\hline C & & & \\
\hline $\mathrm{H}$ & & & \\
\hline 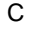 & & & \\
\hline C & & & \\
\hline C & 6.0 & & \\
\hline C & 000 & & 0000 \\
\hline C & 7.27 & 2000 & 6000 \\
\hline C & & & \\
\hline $\mathrm{H}$ & & & \\
\hline 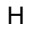 & & & \\
\hline a & & & \\
\hline $\mathrm{H}$ & & & \\
\hline t & & & \\
\hline ; & -3. & -2 & \\
\hline C & -3 & -1.7 & -1 \\
\hline , & -3.8 & 3000 & 1000 \\
\hline 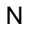 & -4.6 & & \\
\hline $\mathrm{H}$ & & & \\
\hline $\mathrm{H}$ & & & \\
\hline $\mathrm{r}$ & & & \\
\hline r & & & \\
\hline 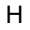 & & & \\
\hline † & -4 & & \\
\hline t & 0 & & \\
\hline $\mathrm{H}$ & -4.6 & & -0 . \\
\hline $\mathrm{H}$ & -5.5 & -0. & -0 . \\
\hline V & -1.6 & & \\
\hline C & & & \\
\hline C & & & \\
\hline C & & & \\
\hline C & & & \\
\hline C & & & \\
\hline $\mathrm{H}$ & & & \\
\hline $\mathrm{H}$ & -1.7 & -3.9 & \\
\hline $\mathrm{H}$ & & & \\
\hline $\mathrm{H}$ & & & \\
\hline C & 000 & & \\
\hline $\mathrm{H}$ & & & \\
\hline & & & \\
\hline & & & \\
\hline & & & \\
\hline & & & \\
\hline & & & \\
\hline & & & \\
\hline & & & \\
\hline & & & \\
\hline
\end{tabular}




\begin{tabular}{|c|c|c|c|c|c|c|c|}
\hline $\begin{array}{l}\mathrm{H} \\
\mathrm{H} \\
\mathrm{H} \\
\mathrm{S} \\
\mathrm{C} \\
\mathrm{H} \\
\mathrm{H} \\
\mathrm{H} \\
\mathrm{H} \\
\mathrm{H}\end{array}$ & $\begin{array}{r}5.889918000 \\
6.503980000 \\
8.116460000 \\
9.309666000 \\
10.053663000 \\
10.018407000 \\
9.565087000 \\
11.097735000 \\
8.429743000\end{array}$ & $\begin{array}{r}0.808109000 \\
3.831351000 \\
1.791462000 \\
-1.104421000 \\
0.090266000 \\
-0.263752000 \\
1.064060000 \\
0.186719000 \\
3.319992000\end{array}$ & $\begin{array}{r}2.440637000 \\
-1.435193000 \\
1.987342000 \\
0.333018000 \\
-0.803839000 \\
-1.836092000 \\
-0.718034000 \\
-0.498300000 \\
0.050656000\end{array}$ & $\begin{array}{l}\mathrm{H} \\
\mathrm{H} \\
\mathrm{H} \\
\mathrm{H} \\
\mathrm{S} \\
\mathrm{C} \\
\mathrm{H} \\
\mathrm{H} \\
\mathrm{H}\end{array}$ & $\begin{array}{r}5.310003000 \\
4.728782000 \\
7.700382000 \\
7.111890000 \\
9.279134000 \\
9.992622000 \\
9.944400000 \\
9.495877000 \\
11.040430000\end{array}$ & $\begin{array}{r}0.211139000 \\
-2.640349000 \\
0.326782000 \\
-2.533655000 \\
-1.088478000 \\
0.190265000 \\
-0.092249000 \\
1.150368000 \\
0.277181000\end{array}$ & $\begin{array}{r}-2.491303000 \\
0.678235000 \\
-1.965683000 \\
1.209785000 \\
0.150081000 \\
-0.912196000 \\
-1.965829000 \\
-0.751405000 \\
-0.616637000\end{array}$ \\
\hline & & & & & & & \\
\hline \multicolumn{4}{|c|}{$s y n-[($ up $-s c) \cdot($ gauche $-s t)-(E)-\mathbf{1} \cdot(E)-\mathbf{2} \mathbf{a}]\left(\mathrm{S}_{1}\right.$ state $)$} & \multicolumn{4}{|c|}{$s y n-[(u p-s c) \cdot(t g-s t)-(E)-\mathbf{1} \cdot(E)-\mathbf{2 b}]\left(\mathrm{S}_{1}\right.$ state $)$} \\
\hline \multicolumn{4}{|c|}{ Cartesian coordinates $(\AA)$ : } & \multicolumn{4}{|c|}{ Cartesian coordinates $(\AA)$ : } \\
\hline C & -0.214950000 & -1.959377000 & 1.806354000 & C & -1.117111000 & -2.024204000 & 2.159320000 \\
\hline C & 1.129831000 & -1.644253000 & 1.794142000 & C & 0.253043000 & -1.844409000 & 2.010956000 \\
\hline C & 1.587286000 & -0.362729000 & 1.413611000 & C & 0.788296000 & -0.607216000 & 1.607802000 \\
\hline C & 0.635624000 & 0.596102000 & 0.983414000 & C & -0.106844000 & 0.456032000 & 1.322230000 \\
\hline C & -2.971366000 & -2.389382000 & 2.048321000 & C & -3.914228000 & -2.272952000 & 2.443359000 \\
\hline $\mathrm{O}$ & -2.482207000 & -1.158936000 & 1.494248000 & 0 & -3.328950000 & -1.030369000 & 2.035291000 \\
\hline $\mathrm{O}$ & -5.187645000 & -2.154430000 & 1.145446000 & 0 & -5.814520000 & -1.913453000 & 1.074860000 \\
\hline C & -4.439681000 & -2.244229000 & 2.346052000 & C & -5.410856000 & -2.096861000 & 2.417051000 \\
\hline $\mathrm{O}$ & -7.099443000 & -0.601561000 & -0.371674000 & O & -7.137851000 & -0.511632000 & -1.116670000 \\
\hline $\mathrm{C}$ & -7.323782000 & -1.915067000 & 0.111121000 & C & -7.557476000 & -1.740773000 & -0.530935000 \\
\hline C & -6.588310000 & -2.183865000 & 1.404762000 & C & -7.223362000 & -1.824673000 & 0.942332000 \\
\hline 0 & -6.418732000 & 2.174446000 & -0.060408000 & 0 & -6.578209000 & 2.299747000 & -0.855627000 \\
\hline C & -7.687807000 & 1.697346000 & -0.481542000 & C & -7.577721000 & 1.760365000 & -1.698670000 \\
\hline C & -7.967469000 & 0.372273000 & 0.183691000 & C & -8.130641000 & 0.507533000 & -1.065858000 \\
\hline C & -1.154306000 & -0.989223000 & 1.442572000 & C & -1.984599000 & -0.966348000 & 1.904391000 \\
\hline C & -0.710739000 & 0.299756000 & 1.008748000 & c & -1.466012000 & 0.293995000 & 1.491551000 \\
\hline $\mathrm{O}$ & -1.719114000 & 1.150465000 & 0.692328000 & O & -2.393547000 & 1.269356000 & 1.307086000 \\
\hline C & -1.393269000 & 2.476880000 & 0.267474000 & C & -1.917540000 & 2.550550000 & 0.895125000 \\
\hline $\mathrm{C}$ & -2.582179000 & 3.369455000 & 0.516862000 & C & -3.086646000 & 3.500328000 & 0.843868000 \\
\hline O & -3.698831000 & 2.952391000 & -0.242578000 & O & -3.969211000 & 3.110165000 & -0.195187000 \\
\hline C & -4.764621000 & 3.881188000 & -0.118435000 & C & -5.034679000 & 4.034273000 & -0.373235000 \\
\hline C & -6.002585000 & 3.326925000 & -0.775688000 & C & -5.996667000 & 3.473071000 & -1.390336000 \\
\hline $\mathrm{C}$ & 2.996809000 & -0.103233000 & 1.549882000 & C & 2.218939000 & -0.459531000 & 1.570927000 \\
\hline C & 3.664628000 & 1.017794000 & 1.161138000 & C & 2.896798000 & 0.708195000 & 1.312549000 \\
\hline C & 5.079995000 & 1.262888000 & 1.379738000 & C & 4.308771000 & 0.930103000 & 1.497064000 \\
\hline C & 5.665801000 & 2.395280000 & 0.783630000 & C & 4.803008000 & 2.246167000 & 1.344392000 \\
\hline C & 7.021272000 & 2.660206000 & 0.933409000 & C & 6.136632000 & 2.541090000 & 1.580776000 \\
\hline C & 7.817922000 & 1.803901000 & 1.695748000 & C & 7.018703000 & 1.524191000 & 1.957064000 \\
\hline C & 7.247327000 & 0.687253000 & 2.311412000 & C & 6.553751000 & 0.212238000 & 2.091469000 \\
\hline C & 5.897318000 & 0.412832000 & 2.152149000 & C & 5.220335000 & -0.088004000 & 1.861986000 \\
\hline $\mathrm{H}$ & -0.532507000 & -2.950521000 & 2.104751000 & $\mathrm{H}$ & -1.501474000 & -2.984161000 & 2.481827000 \\
\hline $\mathrm{H}$ & 1.854921000 & -2.390679000 & 2.104451000 & $\mathrm{H}$ & 0.926964000 & -2.666606000 & 2.232936000 \\
\hline $\mathrm{H}$ & 0.973827000 & 1.572601000 & 0.658477000 & $\mathrm{H}$ & 0.282362000 & 1.417089000 & 1.010596000 \\
\hline $\mathrm{H}$ & -2.453366000 & -2.596671000 & 2.989822000 & $\mathrm{H}$ & -3.577122000 & -2.521852000 & 3.454922000 \\
\hline $\mathrm{H}$ & -2.782168000 & -3.204527000 & 1.340658000 & $\mathrm{H}$ & -3.614445000 & -3.068086000 & 1.751356000 \\
\hline $\mathrm{H}$ & -4.612432000 & -1.356443000 & 2.970182000 & $\mathrm{H}$ & -5.705506000 & -1.235756000 & 3.034402000 \\
\hline $\mathrm{H}$ & -4.753963000 & -3.130316000 & 2.913332000 & $\mathrm{H}$ & -5.875734000 & -2.997889000 & 2.841189000 \\
\hline $\mathrm{H}$ & -8.396856000 & -2.095581000 & 0.257058000 & $\mathrm{H}$ & -8.636231000 & -1.873293000 & -0.675745000 \\
\hline $\mathrm{H}$ & -6.961244000 & -2.600601000 & -0.661604000 & $\mathrm{H}$ & -7.034637000 & -2.540049000 & -1.064727000 \\
\hline $\mathrm{H}$ & -6.841479000 & -1.430938000 & 2.164372000 & $\mathrm{H}$ & -7.604385000 & -0.944753000 & 1.482621000 \\
\hline $\mathrm{H}$ & -6.868518000 & -3.171775000 & 1.794269000 & $\mathrm{H}$ & -7.699676000 & -2.718837000 & 1.369632000 \\
\hline $\mathrm{H}$ & -8.465834000 & 2.420747000 & -0.203022000 & $\mathrm{H}$ & -8.394164000 & 2.484519000 & -1.832241000 \\
\hline $\mathrm{H}$ & -7.704334000 & 1.574009000 & -1.574018000 & $\mathrm{H}$ & -7.161143000 & 1.525568000 & -2.689522000 \\
\hline
\end{tabular}




\begin{tabular}{|c|c|c|c|}
\hline $\mathrm{H}$ & -7.823958000 & .463108000 & 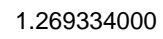 \\
\hline & -9.013539000 & .093229000 & -0.003955000 \\
\hline & -0.563055000 & 2.871469000 & 0.860004000 \\
\hline & 0 & 0 & 00 \\
\hline & -2.8 & & \\
\hline & -2.293040000 & & 000 \\
\hline & -4.9 & 1000 & \\
\hline & -4.494798000 & & 000 \\
\hline & -6.7 & & 000 \\
\hline & -5.80 & & \\
\hline & 820000 & -0.910216000 & \\
\hline & 22000 & 1.809480000 & 000 \\
\hline & 5.04 & 0 & 00 \\
\hline & 7.4 & & 000 \\
\hline & 000 & 00 & 000 \\
\hline & 5.4 & 0 & 00 \\
\hline & -3 & 00 & -2 \\
\hline & -4 & 00 & 000 \\
\hline & & 00 & \\
\hline & & & \\
\hline & & & \\
\hline & -5 & & \\
\hline & & 00 & \\
\hline & & & \\
\hline & -4.73 & 00 & \\
\hline 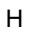 & -5.000 & & \\
\hline & 0000 & 3000 & -2. \\
\hline & 0.274697000 & 000 & -1. \\
\hline & 94000 & 00 & \\
\hline 0 & 096000 & 3000 & -2 \\
\hline C & -0.283459000 & -0.276557000 & -2.229 \\
\hline & -0.689719000 & -2.483908000 & -1.3 \\
\hline $\mathrm{H}$ & -2.771211000 & -3.0 & \\
\hline $\mathrm{H}$ & -2.053787000 & 0.761289000 & $-2 . \varepsilon$ \\
\hline $\mathrm{H}$ & 0.351038000 & 0.546839000 & -2.534 \\
\hline H & -0.359419000 & -3.426903000 & -0.89 \\
\hline & 8.878060000 & & \\
\hline v & & & \\
\hline $\mathrm{H}$ & 1.950278000 & -2.598690000 & \\
\hline & 2.63 & & \\
\hline$\Pi$ & 2.32 & 00 & \\
\hline C & 4.016478000 & -0.797908000 & \\
\hline C & 4.877714000 & .000 & \\
\hline C & 3000 & -1.80 & -0.6 \\
\hline C & 6.233306000 & 000 & -1.74 \\
\hline C & 5.96 & -1.79 & -0. \\
\hline 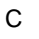 & 718000 & -0.790 & -0. \\
\hline$H$ & 4.448326000 & 1.00 & -2.608 \\
\hline$H$ & 3.997776000 & 6000 & -0.2 \\
\hline & 00 & 00 & -2 \\
\hline & 6.395066000 & -2.578845000 & 0.20489300 \\
\hline & 8.513075000 & -0.903570000 & \\
\hline & 9.221202000 & 0.533918000 & -1.42356900 \\
\hline & 10.288990000 & 0.515130000 & -1.19531600 \\
\hline & 9.085632000 & 0.466809000 & -2.50515300 \\
\hline & 8.790030000 & 1.462004000 & -1.04128700 \\
\hline
\end{tabular}

\begin{tabular}{|c|c|c|c|}
\hline $\mathrm{H}$ & -8.426829000 & 0.710867000 & -0.028077000 \\
\hline $\mathrm{H}$ & -9.016854000 & 0.179758000 & -1.624180000 \\
\hline $\mathrm{H}$ & -1.185336000 & 2.927721000 & 1.617947000 \\
\hline $\mathrm{H}$ & -1.440013000 & 2.471092000 & -0.089993000 \\
\hline $\mathrm{H}$ & -3.613892000 & 3.509318000 & 1.807570000 \\
\hline $\mathrm{H}$ & -2.702156000 & 4.510224000 & 0.648512000 \\
\hline $\mathrm{H}$ & -5.553049000 & 4.198238000 & 0.581349000 \\
\hline $\mathrm{H}$ & -4.639001000 & 4.995529000 & -0.726644000 \\
\hline $\mathrm{H}$ & -6.770628000 & 4.224426000 & -1.603011000 \\
\hline $\mathrm{H}$ & -5.470013000 & 3.248228000 & -2.329683000 \\
\hline $\mathrm{H}$ & 2.779724000 & -1.342731000 & 1.868170000 \\
\hline $\mathrm{H}$ & 2.329829000 & 1.589405000 & 1.018873000 \\
\hline $\mathrm{H}$ & 4.112069000 & 3.033922000 & 1.054875000 \\
\hline $\mathrm{H}$ & 6.494644000 & 3.559863000 & 1.469232000 \\
\hline $\mathrm{H}$ & 7.243207000 & -0.579496000 & 2.369555000 \\
\hline $\mathrm{H}$ & 4.878643000 & -1.113271000 & 1.967930000 \\
\hline $\mathrm{C}$ & -2.622909000 & -1.920881000 & -2.468821000 \\
\hline $\mathrm{C}$ & -3.638380000 & -1.511480000 & -1.406774000 \\
\hline C & -3.675966000 & -0.002743000 & -1.229090000 \\
\hline $\mathrm{N}$ & -4.623594000 & 0.379383000 & -0.155085000 \\
\hline $\mathrm{H}$ & -2.741192000 & -2.977816000 & -2.714585000 \\
\hline $\mathrm{H}$ & -2.766713000 & -1.342808000 & -3.385631000 \\
\hline $\mathrm{H}$ & -3.392863000 & -2.004248000 & -0.457656000 \\
\hline $\mathrm{H}$ & -4.626285000 & -1.869792000 & -1.717260000 \\
\hline $\mathrm{H}$ & -4.007647000 & 0.492414000 & -2.145997000 \\
\hline $\mathrm{H}$ & -2.697192000 & 0.397461000 & -0.953203000 \\
\hline $\mathrm{H}$ & -4.362794000 & -0.070146000 & 0.730969000 \\
\hline $\mathrm{H}$ & -5.586285000 & 0.089747000 & -0.403664000 \\
\hline $\mathrm{H}$ & -4.604745000 & 1.405366000 & -0.031543000 \\
\hline $\mathrm{N}$ & -1.233539000 & -1.725479000 & -2.034503000 \\
\hline C & 1.509658000 & -1.486443000 & -1.412568000 \\
\hline $\mathrm{C}$ & -0.585455000 & -2.729090000 & -1.363777000 \\
\hline $\mathrm{C}$ & -0.548975000 & -0.581683000 & -2.344669000 \\
\hline $\mathrm{C}$ & 0.770553000 & -0.427431000 & -2.041284000 \\
\hline C & 0.737986000 & -2.638282000 & -1.055400000 \\
\hline $\mathrm{H}$ & -1.187881000 & -3.596945000 & -1.119734000 \\
\hline $\mathrm{H}$ & -1.119657000 & 0.178395000 & -2.867276000 \\
\hline $\mathrm{H}$ & 1.252987000 & 0.498613000 & -2.332125000 \\
\hline $\mathrm{H}$ & 1.212707000 & -3.470704000 & -0.545618000 \\
\hline C & 2.899979000 & -1.454947000 & -1.180222000 \\
\hline $\mathrm{H}$ & 3.321383000 & -2.361864000 & -0.750639000 \\
\hline C & 3.752021000 & -0.388687000 & -1.463213000 \\
\hline $\mathrm{H}$ & 3.327678000 & 0.544491000 & -1.828033000 \\
\hline C & 5.168713000 & -0.411596000 & -1.319309000 \\
\hline C & 5.908372000 & 0.782579000 & -1.518733000 \\
\hline C & 5.910380000 & -1.577229000 & -0.969401000 \\
\hline C & 7.279460000 & 0.830916000 & -1.356307000 \\
\hline C & 7.278340000 & -1.535498000 & -0.820281000 \\
\hline C & 7.987282000 & -0.327861000 & -0.992085000 \\
\hline $\mathrm{H}$ & 5.373116000 & 1.686418000 & -1.799026000 \\
\hline $\mathrm{H}$ & 5.399423000 & -2.524383000 & -0.828644000 \\
\hline $\mathrm{H}$ & 7.795946000 & 1.772891000 & -1.505830000 \\
\hline $\mathrm{H}$ & 7.817351000 & -2.441277000 & -0.553867000 \\
\hline$S$ & 9.725585000 & -0.386747000 & -0.745178000 \\
\hline C & 10.229058000 & 1.338987000 & -0.944819000 \\
\hline $\mathrm{H}$ & 11.307258000 & 1.358187000 & -0.774168000 \\
\hline $\mathrm{H}$ & 10.023217000 & 1.699331000 & -1.954698000 \\
\hline $\mathrm{H}$ & 9.737697000 & 1.976271000 & -0.205797000 \\
\hline $\mathrm{H}$ & 8.064133000 & 1.750760000 & 2.145740000 \\
\hline
\end{tabular}

